\title{
WestVirginiaUniversity
}

THE RESEARCH REPOSITORY @ WVU

Graduate Theses, Dissertations, and Problem Reports

2016

\section{Upscaling of sagd process using spectrum-based analysis for heterogeneius reservoirs}

\author{
Vahid Mohammadnia
}

Follow this and additional works at: https://researchrepository.wvu.edu/etd

\section{Recommended Citation}

Mohammadnia, Vahid, "Upscaling of sagd process using spectrum-based analysis for heterogeneius reservoirs" (2016). Graduate Theses, Dissertations, and Problem Reports. 6244.

https://researchrepository.wvu.edu/etd/6244

This Thesis is protected by copyright and/or related rights. It has been brought to you by the The Research Repository @ WVU with permission from the rights-holder(s). You are free to use this Thesis in any way that is permitted by the copyright and related rights legislation that applies to your use. For other uses you must obtain permission from the rights-holder(s) directly, unless additional rights are indicated by a Creative Commons license in the record and/ or on the work itself. This Thesis has been accepted for inclusion in WVU Graduate Theses, Dissertations, and Problem Reports collection by an authorized administrator of The Research Repository @ WVU. For more information, please contact researchrepository@mail.wvu.edu. 


\title{
UPSCALING OF SAGD PROCESS USING SPECTRUM-BASED ANALYSIS FOR HETEROGENEIUS RESERVOIRS
}

\author{
Vahid Mohammadnia \\ Thesis submitted to the West Virginia University \\ in partial fulfillment of the requirements for the degree of \\ Master of Science in \\ Petroleum and Natural Gas Engineering \\ Ali Takbiri Borujeni, Ph.D., Chair \\ Ebrahim Fathi, Ph.D. \\ Samuel Ameri, M.S. \\ Petroleum \& Natural Gas Engineering Department
}

Morgantown, West Virginia

2016

Keywords: SAGD, Heterogeneity, Spectral Representation, Analytical Modeling Copyright 2016 Vahid Mohammadnia 


\title{
Abstract \\ UPSCALING OF SAGD PROCESS USING SPECTRUM-BASED ANALYSIS FOR HETEROGENEIUS RESERVOIRS
}

\begin{abstract}
Vahid Mohammadnia
The main objective for this work is to predict the impact of heterogeneity in the rock and fluid properties on the governing equations in modeling of SAGD process. To serve this purpose, a spectrum-based upscaling approach is applied on the governing equations.

A probability-based analysis is used to include heterogeneity in the fluid and rock properties by assigning random perturbation fields to each property. Furthermore, each property is decomposed into the expectation and perturbation values. Heat diffusion and Darcy equations are upscaled by applying Fourier-Stieltjes transform. The upscaled equations embrace the heterogeneity in permeability and thermal diffusivity and can predict the flow rate and shape of boundary. The upscaled model that includes heterogeneity of reservoir properties is compared with the deterministic solutions (original Butler's model) to quantify the impacts of variation in each property on the SAGD efficiency.
\end{abstract}

The verification and validation of the developed results for the heat equation is done by numerical simulations of models with synthetic heterogeneities; then, a harmonic distribution is assigned to the perturbations of the thermal diffusivity and permeability to calculate the flow rate. In general, there is no standard analytical correlation between permeability and thermal diffusivity of the porous media. In order to investigate the impact of these properties on SAGD, two different cases of positive and negative log-linear correlation (c) between permeability and thermal diffusivity are considered. The results show that in a heterogeneous porous medium with both positive and negative correlations between permeability and thermal diffusivity, the perturbation of thermal diffusivity impairs the efficiency of the SAGD process and its effect is more significant than permeability perturbations. Unlike thermal diffusivity, heterogeneity of permeability, depending on the nature of its correlation with thermal diffusivity, may have either negative or positive effect on SAGD process. 
This work is one of the first attempts to modify the Butler's model by upscaling the governing equations in SAGD process. The new developed equations pave the way of a theoretical solution to a more accurate SAGD modeling. 


\section{DEDICATION}

I dedicate this thesis to my parents who supported me from thousand miles away through the two years of doing this research

Without them none of this success would be possible 


\section{Acknowledgment}

I would first like to thank my thesis advisor Dr. Ali Takbiri who consistently welcomed me to implement my ideas on this thesis and directed me in the right the direction whenever he thought I needed it.

I would also like to acknowledge Dr. Ebrahim Fathi as the second reader of this thesis, and I am gratefully thank him for his very helpful comments on this thesis.

Finally, I must express my deep gratitude to my parents for providing me with constant support and positive encouragement throughout this research. This accomplishment would not have been possible without them. 


\section{Problem Statement}

There is still a huge amount of heavy oil in the world and some are being produced and some in line to be produced after conventional reservoirs are depleted. Steam Assisted Gravity Drainage (SAGD) is one of common enhanced recovery methods to produce heavy oil. An accurate model of this technique is needed to predict the process behavior and take the right managerial decisions that become financially beneficial. But creating an accurate model is not an easy task because SAGD deals with a large set of reservoir variables. Heterogeneity in the reservoir is one of the troublesome factors when mimicking the SAGD behavior. Due to the large extent of reservoir and also accessibility limitations to its deep depth, it is practically impossible to measure all parameters required in SAGD modeling. In addition, assuming that a property of a reservoir or fluid has a constant value might generate great simulation errors and lead to huge misunderstanding of the process behavior. To tackle this problem, a couple of numerical methods have been developed to include the reservoir heterogeneity; however, they depend on the availability of large amount of data to run and also are very time consuming.

Aforementioned problems are intrinsic to numerical models but can be addressed if heterogeneity of the reservoir is incorporated in the main governing equations of already developed deterministic SAGD model. In other words, if we can detect the behavior of heterogeneity statistically and show that how this statistical behavior affect the governing equations, there is no need of numerical methods anymore. New model is apparently deterministic but indeed embraces the heterogeneity at the heart of its equations. 


\section{Objective}

The objective for this work is to develop an analytical proxy for SAGD process in heterogeneous reservoirs that is faster and more accurate than the current simulation/modeling techniques. The proposed model is obtained by modifying the current determistic model of SAGD (Butler's Model) so that it gets capable of mimicking the behavior of heterogeneous reservoir. In this project, heterogeneity is defined as a random variable of reservoir properties that makes SAGD a stochastics process. The final objective is to create a proxy model that helps us to analyze this stochastic behavior in a deterministic manner so that the problems of numerical approached are evaded.

To verify the new model, different stochastic processes are considered and results are compared with deterministic case. The new model helps us analyze the stochastic behavior of reservoir when the SAGD process is applied in a straightforward way. 


\section{Table of Contents}

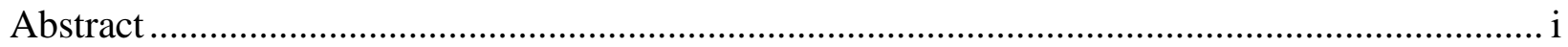

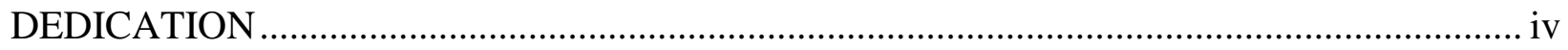

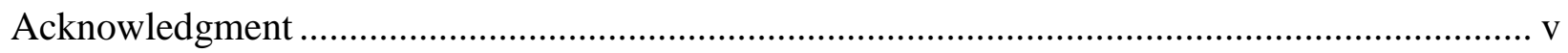

Problem Statement .................................................................................................. vi

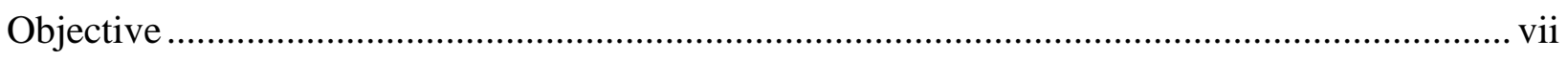

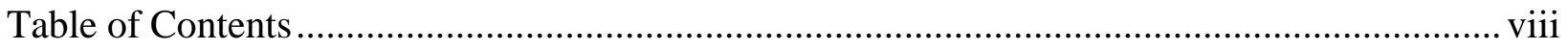

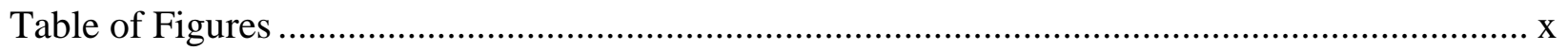

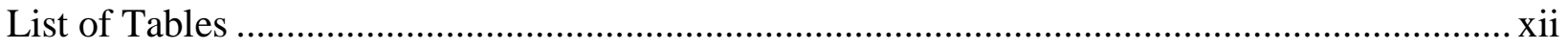

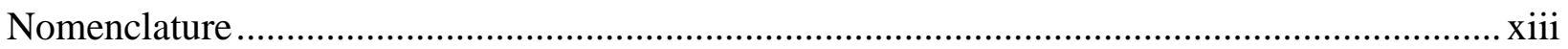

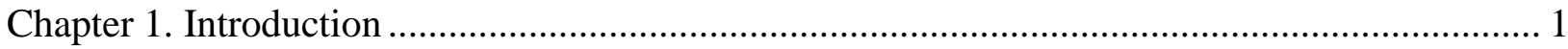

1.1. Geological Distribution of Oil Reserves ................................................................... 2

1.2. Importance of SAGD in North America and US ................................................. 4

1.3. SAGD Process and Butler's Model............................................................................... 4

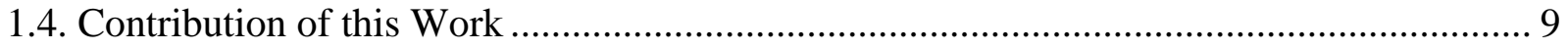

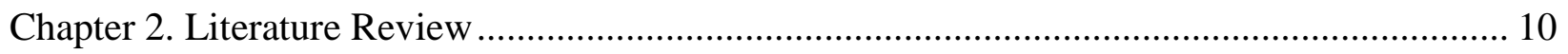

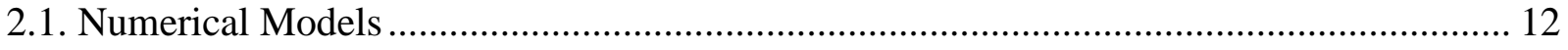

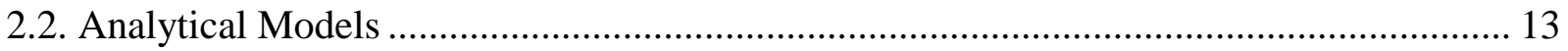

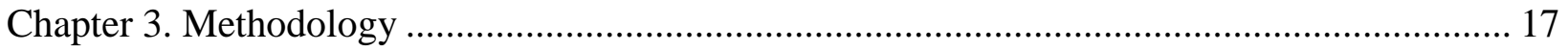

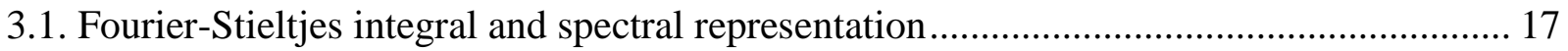

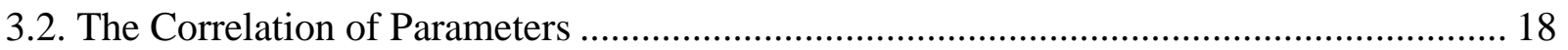

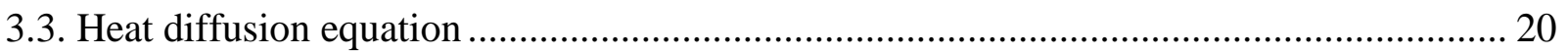

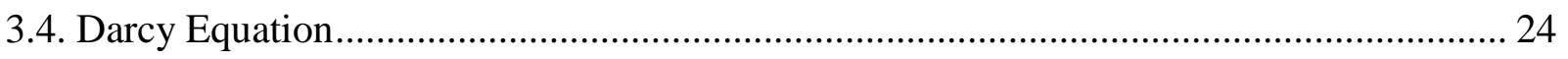

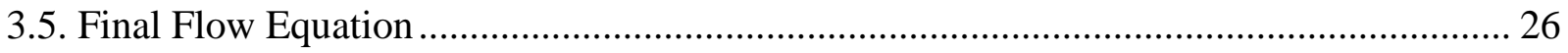




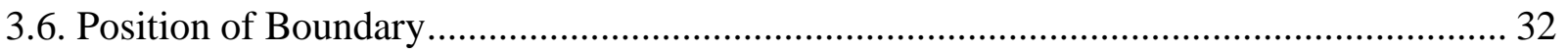

Chapter 4. Verification of Upscaled Thermal Equation .............................................................. 34

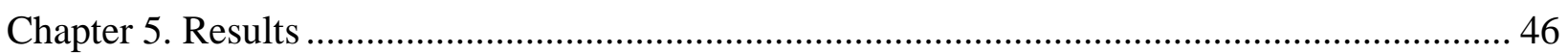

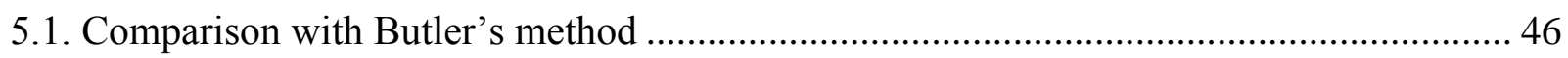

5.1.1. Parameter $\boldsymbol{m}$ calculation....................................................................................... 46

5.1.2. Consistency with Butler's Model ………………....................................................... 47

5.2. Evaluating the developed flow equation for typical values .............................................. 49

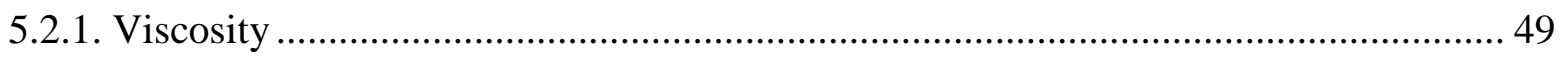

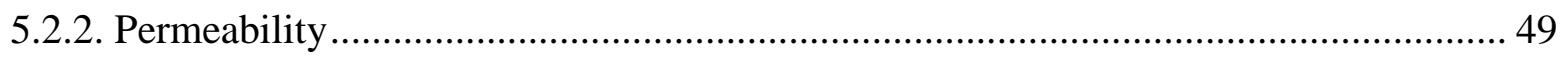

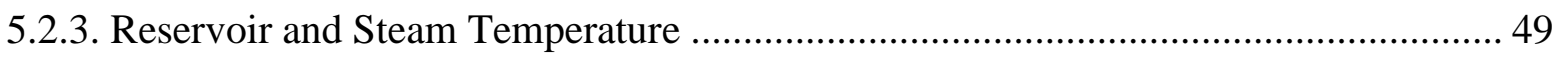

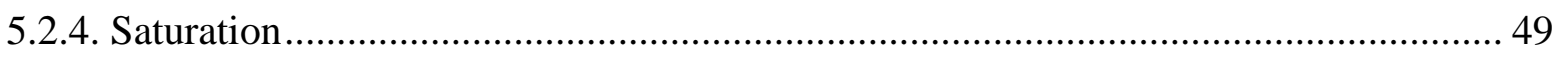

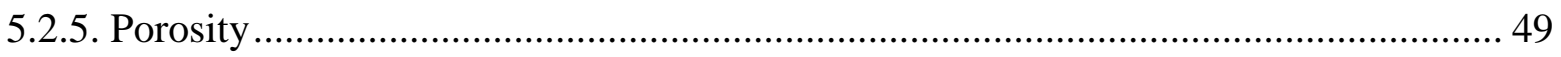

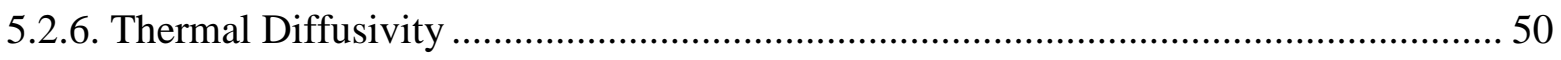

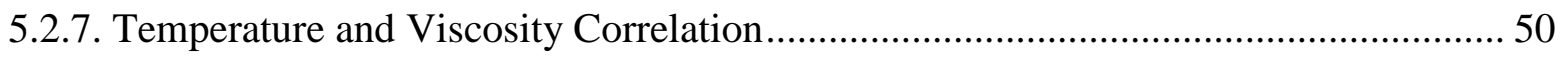

5.2.8. Viscosity of Fluid at Reservoir Temperature ............................................................ 50

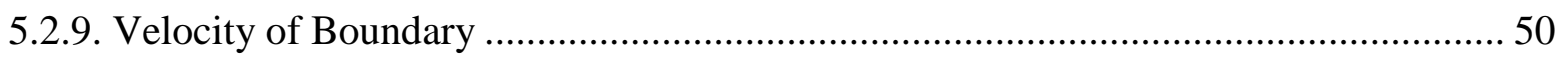

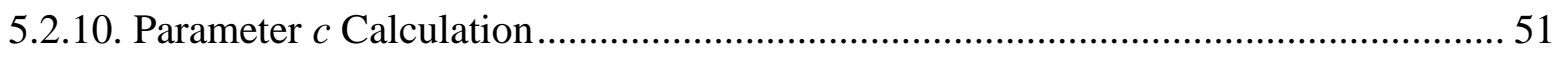

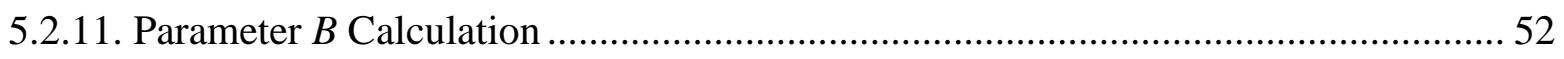

5.2.12. Spectrum of thermal diffusivity ……………….................................................... 52

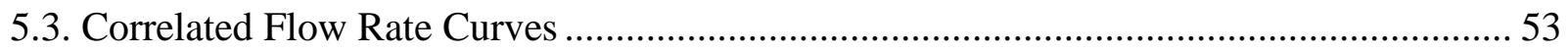

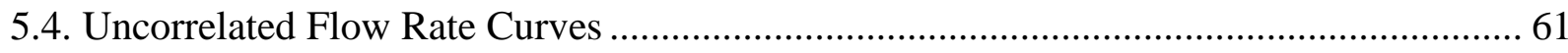

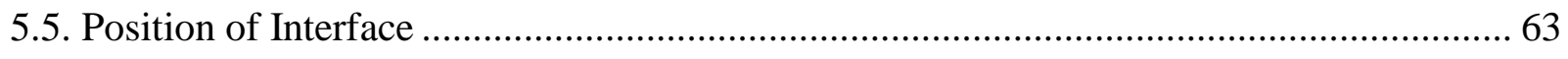

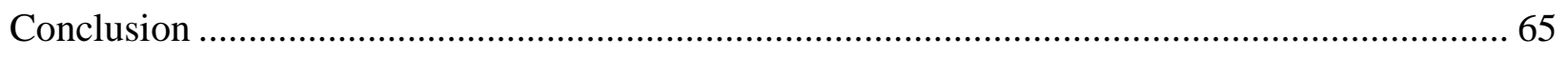

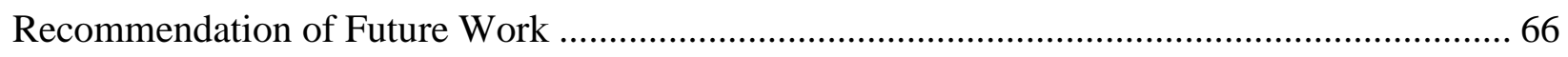

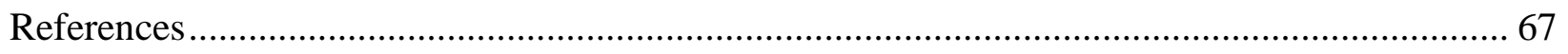




\section{Table of Figures}

Figure 1-1: Stat of recoverable oil in the world ............................................................... 3

Figure 1-2: Steam Chamber Cross-Sectional Representation.............................................. 6

Figure 1-3: SAGD Process Schematic Representation .......................................................... 7

Figure 1-4: Infinitesimal Vertical Section of Interface ...................................................... 7

Figure 2-1: Flow diagram of a simulation path consisting of different models (Azad et al. [27]) 11

Figure 2-2: Schematic of an idealized SAGD chamber during horizontal growth (Courtesy of [12])

Figure 3-1: Flow Diagram of steps to obtain the new SAGD model..................................... 17

Figure 3-2: Cross Sectional View of SAGD along with the expanded view of the edge ............ 26

Figure 4-1: Temperature curves for 50 realizations at a location $1 \mathrm{~m}$ ahead of boundary for $a \alpha=0.7$

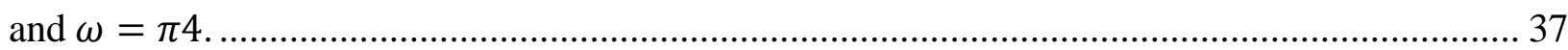

Figure 4-2: Comparison of developed upscaled model with Butler's model and average-based

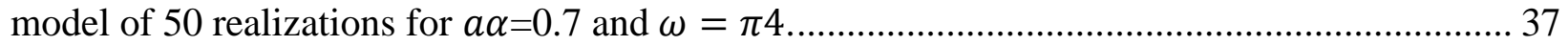

Figure 4-3: Temperature curves for 100 realizations at a location $1 \mathrm{~m}$ ahead of boundary for $a \alpha=0.7$

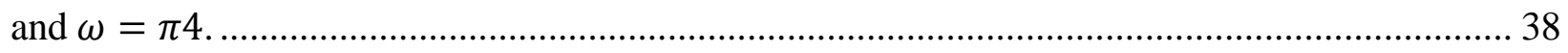

Figure 4-4: Comparison of developed upscaled model with Butler's model and average-based

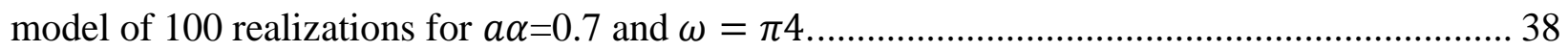

Figure 4-5: Comparison of developed upscaled model with Butler's model and average-based

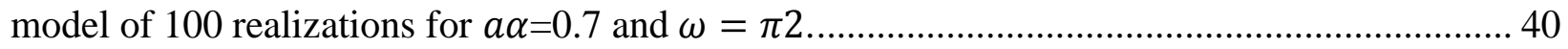
Figure 4-6: Comparison of developed upscaled model with Butler's model and average-based

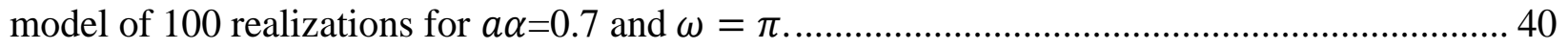
Figure 4-7: Comparison of developed upscaled model with Butler's model and average-based model of 100 realizations for $a \alpha=0.7$ and $\omega=3 \pi 2$.

Figure 4-8: Comparison of developed upscaled model with Butler's model and average-based model of 100 realizations for $a \alpha=0.7$ and $\omega=2 \pi$.

Figure 4-9: Comparison of developed upscaled model with Butler's model and average-based model of 100 realizations for $a \alpha=0.6$ and $\omega=\pi \ldots$ 42 
Figure 4-10: Comparison of developed upscaled model with Butler's model and average-based model of 100 realizations for $a \alpha=0.8$ and $\omega=\pi$.

Figure 4-11: Comparison of developed upscaled model with Butler's model and average-based model of 100 realizations for $a \alpha=0.9$ and $\omega=\pi$.

Figure 4-12: Comparison of developed upscaled model with Butler's model and average-based model of 100 realizations for $a \alpha=0.6, \omega=\pi$ and $U=0.8 \times 4.76 \times 10-7 \mathrm{~ms}$.

Figure 4-13: Comparison of developed upscaled model with Butler's model and average-based model of 100 realizations for $a \alpha=0.6, \omega=\pi$ and $U=1.2 \times 4.76 \times 10-7 \mathrm{~ms}$. 44 Figure 4-14: Comparison of developed upscaled model with Butler's model and average-based model of 100 realizations for $a \alpha=0.6, \omega=\pi$ and $U=1.4 \times 4.76 \times 10-7 \mathrm{~ms}$. 45 Figure 4-15: Comparison of developed upscaled model with Butler's model and average-based model of 100 realizations for $a \alpha=0.6, \omega=\pi$ and $U=16 \times 4.76 \times 10-7 \mathrm{~ms}$. 45

Figure 5-1: Boundary value of $m$ for different fluid oil [2]. 46 Figure 5-2: Heterogeneous properties are assumed to be the sum of their mean and harmonic perturbation. 54

Figure 5-3: Effect of heterogeneities on the flow rate for positive correlation between $\mathrm{k}$ and $\alpha .57$ Figure 5-4: Effect of heterogeneities on the flow rate for negative correlation between $\mathrm{k}$ and $\alpha 57$ Figure 5-5: Values of AA against reservoir heterogeneities for positive correlation .................. 58 Figure 5-6: Values of AA against reservoir heterogeneities for negative correlation ................. 58 Figure 5-7: Flow rate in SAGD for positive correlation...................................................... 59 Figure 5-8: Produced flow rate in SAGD for negative correlation....................................... 59 Figure 5-9: Effect of heterogeneities on the flow rate against constant values of $\mathrm{c}$ for positive correlation 60

Figure 5-10: Effect of heterogeneities on the flow rate against constant values of c for negative correlation 60

Figure 5-11: Effect of permeability heterogeneities on flow rate with no thermal diffusivity perturbation

Figure 5-12: Effect of diffusivity heterogeneities on flow rate with no thermal permeability perturbation 62

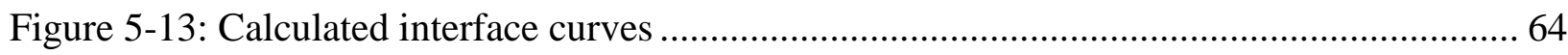

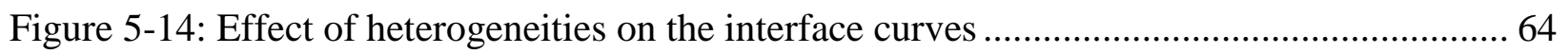




\section{List of Tables}

Table 1-1: Regional distribution of estimated technically recoverable heavy oil and natural bitumen in billions of barrels (BBO) ................................................................................ 3 


\section{Nomenclature}

h: Thickness of the Producing Zone

U: Velocity of boundary

$\mathrm{T}_{\mathrm{R}}$ : Reservoir Temperature

$\mathrm{T}_{\mathrm{s}}$ : Steam Temperature

$\alpha$ : Thermal diffusivity

v: Kinematic Viscosity

$\mathrm{S}=$ Saturation

$\mathrm{q}=$ Flow rate

$\mathrm{a} \alpha=$ Relative magnitude of thermal diffusivity perturbations

$\mathrm{a}_{\mathrm{f}}=$ Relative magnitude of permeability perturbations

k: Permeability

$\omega=$ Angular frequency of perturbations

c: Thermal diffusivity and permeability correlation constant

$S(\omega)=$ Spectrum of perturbations

$R(\tau)=$ Correlation of perturbations

$\beta=$ Temperature and viscosity correlation constant 


\section{Chapter 1. Introduction}

A huge amount of heavy oil remains still untouched throughout the world, about 30 countries have recoverable heavy oil resources led by Canada and Venezuela owning the biggest. Improving the recovery methods and trying to make a reasonably accurate model that is an influential factor in taking the right managerial and operational decisions when dealing with these unconventional resources. Oil fluid can be classified based on API gravity as:

Light oil: If the oil has the API gravity at least 22, it is classified as light oil.

Heavy oil: It is defined as any petroleum liquid having the maximum API gravity of 22 .

Extra heavy oil: World Energy Council defines extra heavy oil as crude oil having a gravity of less than 10 .

Bitumen: Natural bitumen is also called oil sands or tar sands. It typically has a gravity lower than 10. According to US geological Survey, it is distinguished as extra-heavy oil with a higher viscosity. Extra-heavy oil and natural bitumen and differ in the degree by which they have been degraded for the original conventional oils by bacteria.

In addition to dissimilarity in density, heavy oil has a substantial contents of oxygen, nitrogen, and sulfur compounds and heavy metal containments (according to USGS) compared with conventional oil. In spite of abundance, heavy oil and bitumen only accounted 3 billion of 25 billion barrels of crude oil produced in 2000 (USGS). The high cost of production, transportation and upgrading is the main inhibiting factor of industry demand of heavy oil. A State Department review also released that demand for the oil sands fuel would decrease if oil prices were below $\$ 65$ a barrel, as moving oil by rail is more expensive than using a pipeline ${ }^{1}$. However, geological distribution, abundance, quality and price of these types of crude oil will determine the market demand of heavy oil in the future.

${ }^{1}$ The New York Times, November 6, 2015 


\subsection{Geological Distribution of Oil Reserves}

In Figure 1-1 and Table 1-1, the distribution of heavy oil (including extra heavy oil) and light oil is shown worldwide (According to US geological Survey). Technically recoverable oil in known heavy oil and natural bitumen accumulations is almost equal to light oil reserves (API gravity greater than $22^{\circ}$ ) in known conventional accumulations. BBO, billion barrels of oil.

The Western Hemisphere includes 69 percent of the world's technically recoverable heavy oil (including extra-heavy oil) where 61 percent is only on southern America. The Western Hemisphere has also 82 percent of the recoverable natural bitumen whereas the Eastern Hemisphere has about 85 percent of the world's light oil reserves.

Each of extra-heavy and bitumen categories are dominated by a large accumulation. Venezuelan Orinoco heavy-oil belt is the largest extra-heavy oil accumulation, which contains 90 percent of the world's extra-heavy oil. Eighty one percent of the known recoverable bitumen is in the Alberta, Canada. Totally, the two reserves represent about 3,600 billion barrels of oil in place equivalent to resources around 830 billion barrels. Venezuela and Canada together produces $93 \%$ of the global heavy oil and oil sands with more than two third produced in Canada.

The U.S. bitumen accumulations are mostly in Utah but none of them are being produced commercially, although, they are estimated to contain 6.1 billion barrels of recoverable bitumen. Three of 6 of main fields in California are heavy oil fields including: Midway-Sunset, Kern River and South Belridge that each has already produced more than 1 billion barrels. The density if oil in these reservoirs are typically between 10 and 15. The Steam Stimulation methods are very popular among other ways of production methods in these fields [2].

Of the 35 billion barrels of heavy oil estimated to be recoverable in North America, about 7.7 billion barrels belong to known producing accumulations in the lower 48 States, and 7 billion barrels are in the North Slope of Alaska (U.S. Geological Survey). 


\section{Stat of oil recoverable reserves}

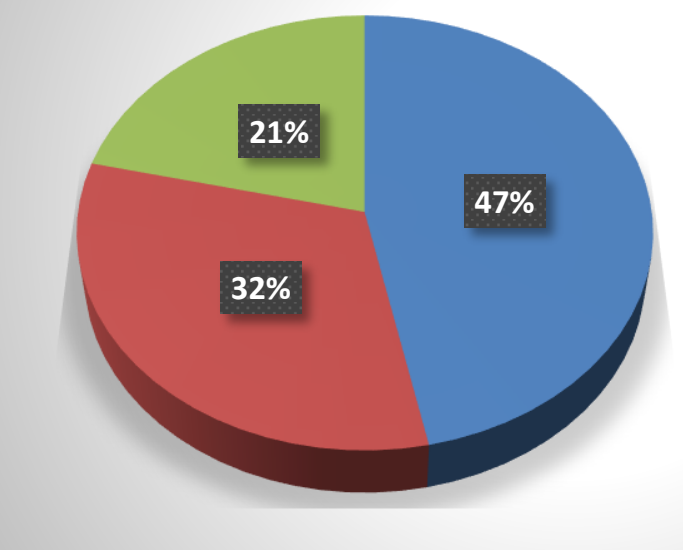

- Conventional (light) oil reserves $=952 \mathrm{BBO}$

Natural bitumen resources $=651 \mathrm{BBO}$

- Heavy/extra heavy oil resources $=651 \mathrm{BBO}$

Figure 1-1: Stat of recoverable oil in the world

Table 1-1: Regional distribution of estimated technically recoverable heavy oil and natural bitumen in billions of barrels (BBO).

\begin{tabular}{|l|l|l|}
\hline & $\begin{array}{c}\text { Heavy Oil } \\
\text { Technically } \\
\text { recoverable BBO }\end{array}$ & \multicolumn{1}{|c|}{$\begin{array}{c}\text { Bitumen } \\
\text { Technically } \\
\text { recoverable BBO }\end{array}$} \\
\hline North America & 35.3 & 530.9 \\
\hline South America & 265.7 & 0.1 \\
\hline Africa & 7.2 & 43 \\
\hline Middle East & 78.2 & 0.0 \\
\hline Asia & 29.6 & 42.8 \\
\hline Russia & 13.4 & 33.7 \\
\hline Western Hemi & 301 & 531 \\
\hline Eastern Hemi & 128.4 & 119.5 \\
\hline Worldwide & 429.4 & 650.5 \\
\hline
\end{tabular}




\subsection{Importance of SAGD in North America and US}

SAGD and Cyclic Steam Stimulation (CSS) are the two main thermal in situ processes used in the oil sands(bitumen) in Geological formation sub-units, such as Grand Rapids Formation, Clearwater Formation, McMurray Formation, General Petroleum Sand, Lloydminster Sand, of the Manville Group, a Stratigraphic range in the Western Canadian Sedimentary Basin [6].

The largest oil import in Unites States comes from Canada, about 35\% of all nationwide imports [7]. Most of the Canada's recent production comes from Alberta's huge oil sands reserves. Much of the future oil production in Alberta is expected to be from SAGD that accentuates the importance of this type of recovery method especially in the near future to United States.

\subsection{SAGD Process and Butler's Model}

Steam assisted gravity drainage (SAGD) can be considered as the advanced version of steam stimulation. It first developed by Roger Butler and his colleagues in Imperial Oil in the late 1970s [1]. SAGD is an enhanced recovery method based on steam injection coupled to horizontal well technology. Common type of SAGD consists of two horizontal wells, one drilled almost at the bottom of reservoir and second is drilled typically 5-10 $\mathrm{m}$ above [3]. Initially, the cold heavy crude is very immobile and needs to be heated to get off from the sluggish mood. The steam is injected into both wells to preheat the highly viscous crude and make the reservoir ready for SAGD. The steam injected to the injector creates a steam chamber that moves upward and forward and sweeps down the heavy oil toward the producer wells. In the initial rising period, the chamber goes up and reaches the overburden and then spreads laterally. This phase is known as the horizontal steam chamber growth phase [23]. The heat is transformed from steam to heavy oil, reduces crude's viscosity and the gravity dominates viscous forces acting upon fluid. This makes the fluid and condensed water drain along the interface into the production well. A small pressure differential, close to gravity head differential is desirable between injection and production wells. Continuous shale barriers may impede the process efficiency, but small, discontinuous shale barriers may not have a significant effect (Sharma et al.[5]). 
With invention of horizontal wells, sweep efficiency was improved, the recoverable reserves was increased and fewer number of wells were required. A 2000-4000-ft long horizontal well can replace several vertical wells in SAGD process (Sharma [5]).

Butler was the first one who modeled the SAGD. The basic assumptions made in his model was considering a 1-D quasi-steady distribution of heat ahead of the steam chamber. This, along with Darcy’s law consists the SAGD bitumen flow expression as derived by Butler.

Two dimensional test done by Chung et al. [24] on a scaled model showed that the efficiency of SAGD is independent of well spacing, however, Sharma et al. [5] achieved better results with close well spacing in the simulation of SAGD on Ugnu reservoir.

One of the obstacle sin SAGD is the low steam injectivity in tar and heavy oil reservoirs. Electrical heating offers a viable solution. It is used as a precursor to steam injection. Another advantage of the EP-SAGD is its relatively low sensitivity to the shale presence (Glandt et al.[25]).

Diversion or steam breakthrough, is a problem that arises in numerous wells due to sand erosion or plugging of the slotted liners, which inhibits continuous production. Swellable packers can be installed in conjunction with screens or slotted liner to spread steam and establish zonal isolation. In the case of steam breakthrough, swellable packers can be operated to separate the affected zones. This intervention technique will contribute to efficient continuous production, elimination of sand production and steam breakthrough (Books et al. [26]).

Temperature ahead of boundary for a steady-state distribution is given by Eq. 1-1:

$$
\frac{T-T_{R}}{T_{S}-T_{R}}=e^{-\frac{U \xi}{\alpha_{e q}}}
$$

Where the variables represent the following properties (Figure 1-4):

$T$ : Temperature at a location with $\xi m$ ahead of boundary $\left(\mathrm{C}^{\circ}\right)$

$T_{R}$ : Temperature at reservoir condition $\left(\mathrm{C}^{\circ}\right)$

$T_{S}$ : Steam Temperature $\left(\mathrm{C}^{\circ}\right)$

$\alpha$ : Thermal diffusivity $\left(\mathrm{m}^{2} / \mathrm{s}\right)$ 


\section{$U:$ Velocity of interface $(\mathrm{m} / \mathrm{s})$}

Since Butler's model, a couple of other models were developed that if driven analytically, they were fundamentally similar to Butler's original model. Each of these models cover a specific aspect of SAGD, but regardless of their respective restrictions, each can be utilized as a proxy model. Any mathematical or statistical function that can mimic the behavior of a process or reservoir is called proxy model (Azad et al.[27]).

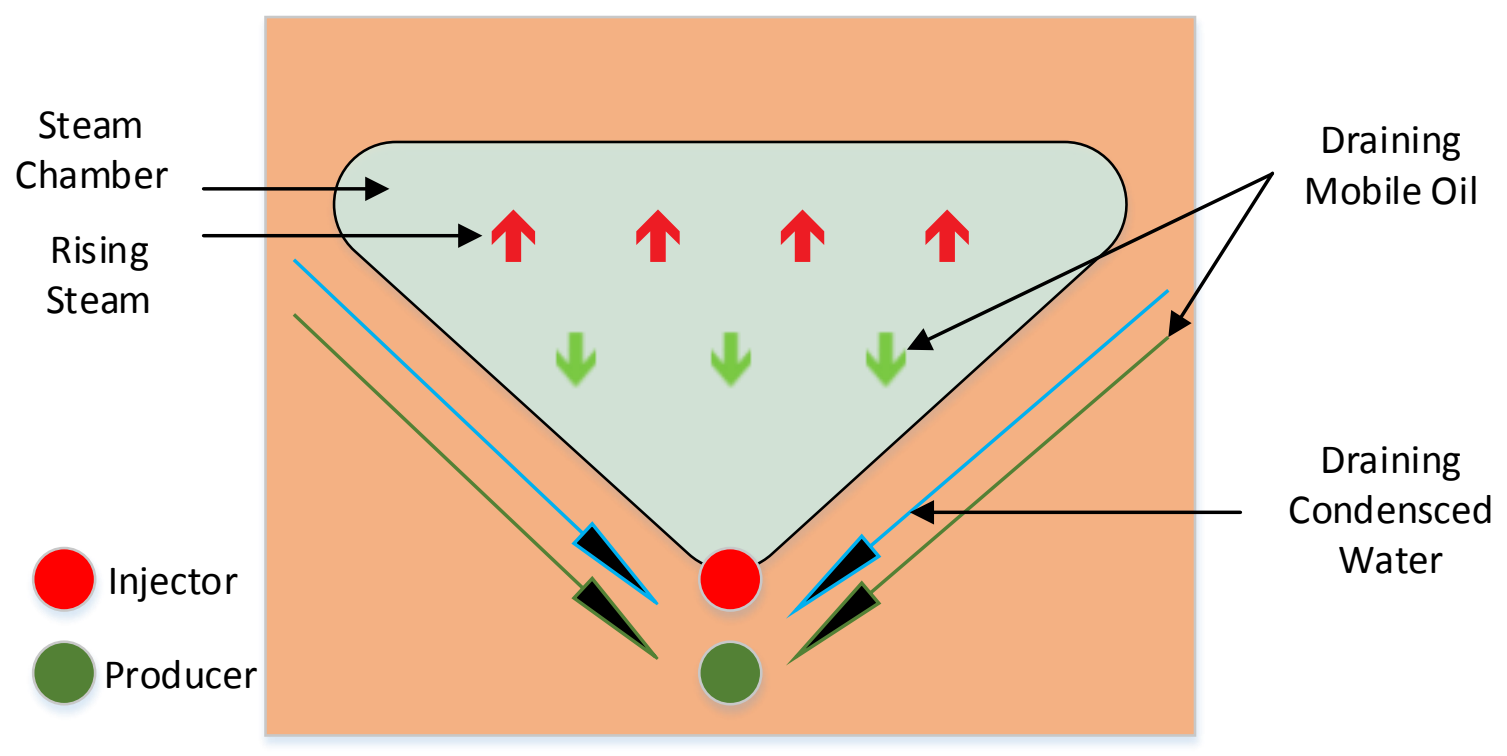

Figure 1-2: Steam Chamber Cross-Sectional Representation 


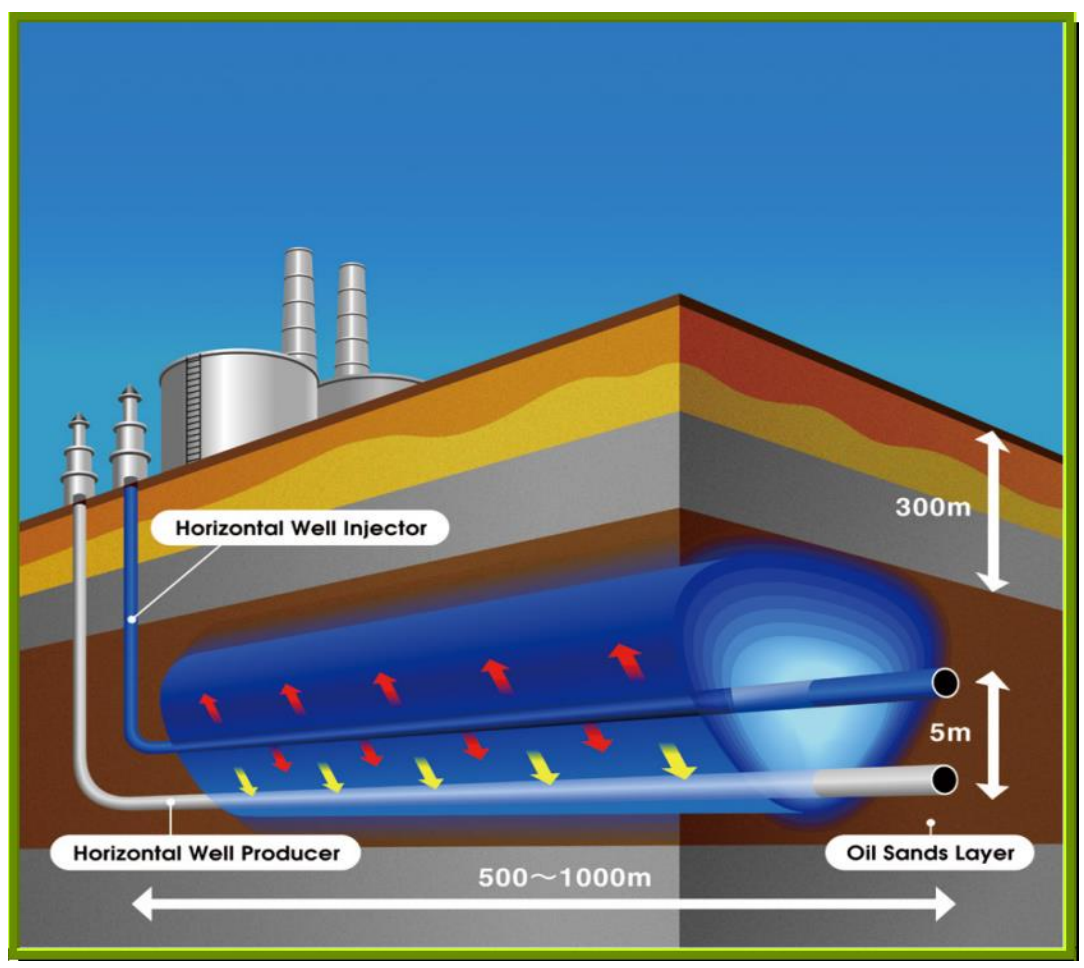

Figure 1-3: SAGD Process Schematic Representation ${ }^{2}$

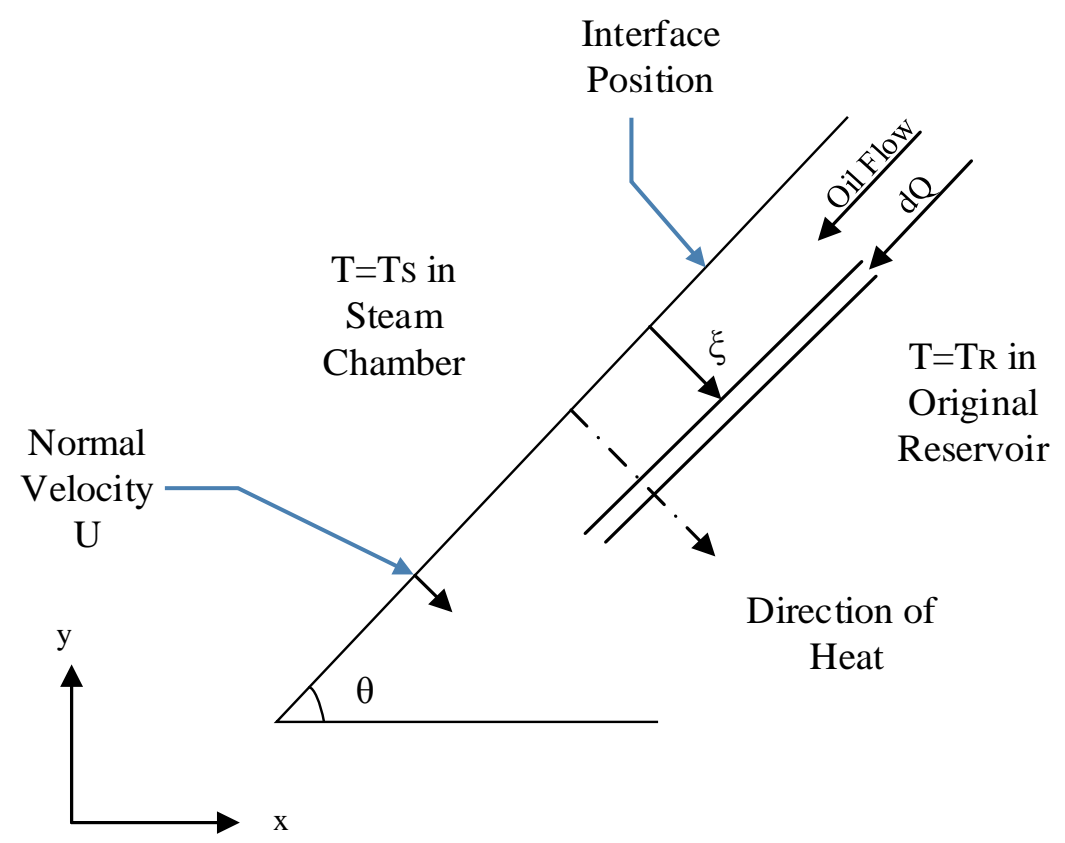

Figure 1-4: Infinitesimal Vertical Section of Interface

${ }^{2}$ Picture curtesy of Japan and Canada Oil Sands Company (Jacos) 
Based on Butler's equation, SAGD bitumen flowrate can be expressed as Eq. 1-2:

$$
q_{o}=\sqrt{2 \phi \Delta S_{o} \frac{K g \alpha h}{m v_{s}}}
$$

Where

$$
m=\left[v_{S} \int_{T_{R}}^{T_{S}}\left(\frac{1}{v}-\frac{1}{v_{R}}\right) \frac{d T}{T-T_{R}}\right]^{-1}
$$

Other parameters in Eq. 1-2 are represented of:

q: Produced flow rate per unit length $\left(\mathrm{m}^{2} / \mathrm{s}\right)$

$\phi:$ Porosity

$K$ : Permeability $\left(\mathrm{m}^{2}\right)$

$v_{S}:$ Viscosity of oil in steam temperature

$\Delta S_{o}$ : Mobile oil saturation, difference of residual and initial oil saturation

Parameter $\mathrm{m}$ is a function of viscosity and temperature characteristic of oil in addition to the reservoir and steam temperature. It can be simply assumed to be a property of the oil that is defined as the integral of Eq. 1-3 and a function of $T_{S}$ and $T_{R}$. This parameter does not change rapidly with $T_{S}$ and $T_{R}$. Thus, in many applications, it can be assumed constant. Eq. 1-2 suggests a simple method to increase the efficiency of SAGD by increasing parameters such as increasing the steam temperature or decreasing the residual saturation.

Butler's equations were derived for a completely homogenous medium and reservoir heterogeneities such as permeability and thermal diffusivity variations were not incorporated in the developed equations. Although numerically based modeling of SAGD process in heterogeneous reservoirs is capable of incorporating heterogeneities and complicated initial and boundary conditions, they fail to give a general insight, as each configuration is treated independently and many runs of simulations may be required to discover the independence of key 
variables to inputs. A large amount of time-consuming effort may be required to treat the situations that do not have analytical solutions.

Moreover, computer codes used in numerical techniques are prone to human error which is usually obscured in long and complicated codes. But the analytical solutions are stated in an accurate form that can be easily rechecked.

\subsection{Contribution of this Work}

In this project, using spectrum-based analysis, we will try to quantify the impact of medium heterogeneities by developing an analytical solution that is capable of incorporating the input perturbations into a standard formulation that exclusively states the effects of input covariance on the key output.

A probability-based analysis is used to include heterogeneity in SAGD modeling by assigning a random variable field to each of varying properties. The effects of heterogeneity are largely distributed over the reservoir. All the basic governing equations that are based on deterministic but inaccurate presumption of fluid and media, makes the results deviate from reality. This concern is investigated in this research by applying Fourier-Stieltjes transform on the governing equations and introducing a new set of equations that embraces the heterogeneity in a straightforward way. The new developed equations pave the way of a theoretical solution to a more accurate SAGD modeling [2]. 


\section{Chapter 2. Literature Review}

In this chapter, a review if the different upscaling approaches of SAGD process is presented. In general, the published models for SAGD in the literature are divided into two groups of numerical and analytical approaches.

Data-driven models use numerical methods to set up a proxy of the real process or reservoir. They require reliable sets of data to run but have the benefit of applicability in a wide range of applications and can incorporate broad sets of input variables and complicated geometries; however, they usually fail to provide a clear intuition of underlying physics of the process. In return, analytical methods use universal formulations to model the underlying physical processes. These models are however valid for specific applications. The solutions to the analytical models have the advantage of offering a general theoretical development without specifying an explicit form for the heterogeneity, in contrast to the numerical solutions in which the explicit from of heterogeneity should be specified. Analytical approaches also have the merit of stating the assumptions and approximations evidently through a precise mathematical description of the problem. In contrary, the nature of approximation in numerical methods, is hidden in the elaborately and usually arbitrarily written computer codes.

Furthermore, analytical approaches provide us with a quick and easy access to solutions through their explicit and mathematical nature whereas personalized and intricate codes are run only on some specific platforms or software that inhibits the accessibility. One of the distinguished advantage of numerical methods is their general application on a great variety of problems. Although this is the right statement, in practice a huge and time-consuming modification on codes is necessary to extent it to new problems.

There is no accurate solution to stochastic partial equations. Choosing the appropriate approach, highly depends on the type of usage and target of the results and also type of data that is available or can be reasonably collected in future. Some applications seek for a general scientific conception whereas others might use the results to include them in their specific practical decision. In short, there is no unique approach that is capable of answering all the questions and clarify all the vague 
parts of the problem, but through an interconnected comparison between the results gathered by different methods, the intended application and concept can be interpreted.

Due to the lack of reliable data, during the development of a SAGD project, there is a lot of uncertainties depending on which stage of the project the modeling is required. In the initial steps of project planning, due to uncertainties of reservoir properties and also lack of time to take the appropriate managerial decision, numerical simulators are not efficient to be used. Instead, proxy models can be used to serve this purpose. But with moving forward in a simulation path, the amount of uncertainties decrease and finer models can be established (Figure 2-1).

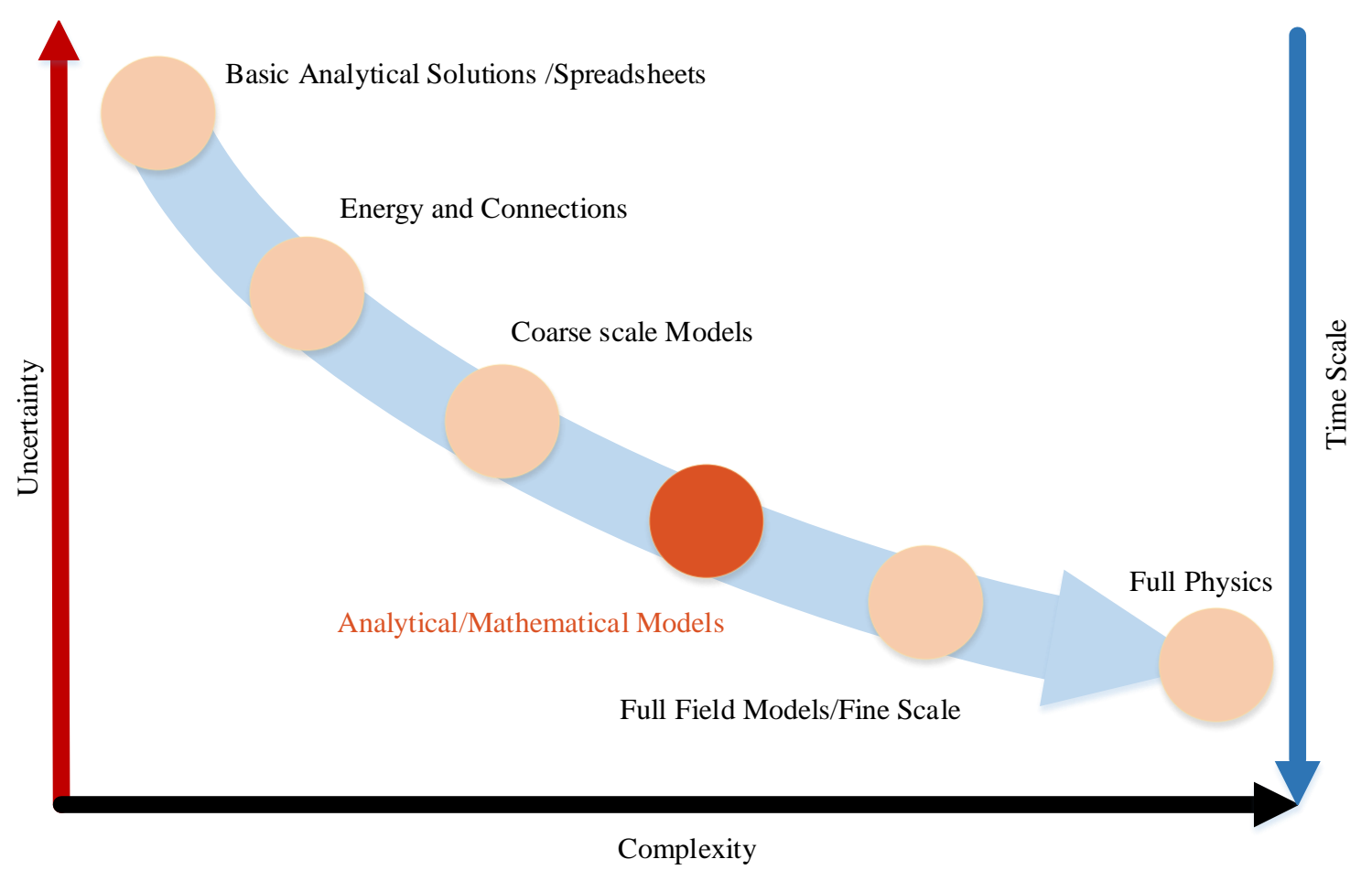

Figure 2-1: Flow diagram of a simulation path consisting of different models (Azad et al. [27])

SAGD process has been modeled both numerically and analytically since 1970's. But the heterogeneity of the reservoirs has not been addressed sufficiently. More specifically, no analytical model has been offered for the SAGD process and all previous works have focused on either numerical or semi-analytical modeling. In the next section, a summary of previous literatures related to this topic on both methods is organized as follows. 


\subsection{Numerical Models}

Based on Butler's theoretical model, Vanegas et al.[3] suggested a method to describe the SAGD process developed between two injection wells. To integrate the heterogeneity effect in their model, they used Monte Carlo Simulation (MCS) and did an efficient transfer of the uncertainty in reservoir and operational parameters through to performance variables such as oil production and steam oil ratio. (Their methodology was actually based on the Roses's thesis [4]). Their method was designed for a confined well pair with the capability of transferring the uncertainty to the model as previously described. An average-based upscaling approach was utilized averaging is to model heterogeneous reservoirs in each timely discretized step which is time consuming and excludes the Butlers' basic model's achievements that were simplicity and quick calculation.

Sharma [5] simulated a SAGD process in Ugnu reservoir, located in the Kuparuki River Unit on the North Slope of Alaska, including SAGD with horizontal injectors, SAGD with vertical injectors, SAGD with electrical preheating (EP-SAGD) and EP-SAGD with vertical injectors. They showed that if the injector chosen to be vertical, heterogeneity slows down the recovery factor and it takes more time to reach the homogeneous recovery factor. This highlights the importance of heterogeneity on determination of well placement in SAGD. They also showed that the placement of production well is more critical than the placement of injection in heterogeneous reservoirs. When the production wells were placed in a lower permeability layer, the recovery factor decreased drastically. However, the heterogeneity was simply incorporated in their model as two different layers having different permeability that is a rough definition of heterogeneity.

Butler et al. [6] studied the SAGD process on multi-layered reservoirs where layers have different permeability. They showed that this heterogeneity in permeability decreases the recovery of heavy oil, because steam tends to accumulate under the layers with lower permeability and thus avoids the heavy oil in upper layers to flow down until the steam gets a way to penetrate upwards and pushes down the heavy oil. They resolved this problem by adding non-condensable gas to the steam that produces gas fingers that rise up into the low permeability layers and displace the oil contain of the layers. Their method on modeling heterogeneity of permeability in multi-layered reservoirs was successful but was valid only for the applications that heterogeneities exist because of the multiple layers rather than the general ones. 
Robinson et al. [7] built a numerical geostatic model of SAGD over a 32-section area of a Fort McMurray oil sand reservoir. They Used Roxar'sirap RMS modeling software to perform geostatic mapping of horizons and populate the petrophysical properties over 3.46 million cell static geological model. They integrated the information gathered form petrophysical logs and core data from 33 wells in the study area to a coherent geostatically based model. Although they fitted the measured data into their model, their method was purely numerical and was valid only for a specific case study and highly dependable on measured data, which might not be always available in project.

Lateral et al.[8] Studied the effect of fluid heterogeneities in terms of variation of heavy oil viscosity in the reservoirs. The heterogeneities in viscosity occur in heavy oil and tar sands due to the microbial degradation of conventional crude oils over geological timescales. Constraints such as oil charge mixing, reservoir-temperature dependent biodegradation rate and supply of water and nutrients to the organisms determine the final distribution of API gravity and viscosity of heavy oil. Their numerical results showed that the oil recovery decreases in the reservoirs with varying viscosities compared with the ones with uniform viscosity. The method they used was numerical and only included the fluid heterogeneities but rock properties were considered uniform as Butler's work.

Chen [9] incorporated the heterogeneities in the reservoirs by including the randomly places shale reservoirs throughout the reservoir. He used STAR software to simulate and analysis the SAGD process. His studies on SAGD was an observation-based analysis of SAGD behavior rather than modeling the SAGD.

\subsection{Analytical Models}

Due to the complexity of reservoirs, only few works have modeled the SAGD analytically. No work has been reported on developing an analytical model that can capture has heterogeneities of permeability and thermal diffusivity in SAGD process. However, other aspects of real life cases were covered by several studies. It has been proved that anisotropy in permeability has a major influence over flow rate in SAGD with horizontal wells (Peaceman ]). Alali et al. [11] developed 
their semi-analytical model using geometric averaging of the vertical and horizontal permeability; nevertheless, they verified the model only over a very small range of cases.

Azom and Srinivasan [12] concentrated on the role of anisotropy of permeability in the reservoirs on the SAGD process. They showed that the effect of vertical permeability on the development of SAGD is time dependent and can be described quantitatively. They proposed two different models for the permeability and incorporated them on the drainage rate formula that already was developed by Butler. They developed their models by resolving the SAGD flow on the direction of resultant gravity head (RGH) and resultant oil discharge (ROD) that led to two different equations for the effective permeability respectively:

$$
\begin{gathered}
K_{\left\{e f f_{R G H}\right\}}=K_{v} \sin ^{2} \theta+K_{h} \cos ^{2} \theta \\
\frac{1}{K_{\left\{e f f_{R G H}\right\}}}=\frac{\sin ^{2} \theta}{K_{v}}+\frac{\cos ^{2} \theta}{K_{v}}
\end{gathered}
$$

Where $\theta$ is the angle that the boundary makes with the horizon (Figure 2-2).

In ROD, the bitumen occurs in the direction tangential to the interface while in $\mathrm{ROH}$, the flow occurs in a direction perpendicular to the equipotential surface.

They included these equations in the Butler's flow rate equation (Eq. 2-3) and developed their own formula in which height of boundary decreases due to the consideration of vertical permeability in comparison with isotropic model.

$$
\bar{q}=\sqrt{2(\alpha) \frac{K_{L} g}{m v_{s}} * \bar{\phi} \Delta S_{o} * h}
$$

Their calculation showed that the anisotropy decreases the efficiency of SAGD. But it seems they missed one concept, they applied their offered effective permeability directly on Eq. 2-3 while to develop this equation itself, isotropy has been assumed for the medium. In other words, all these changes should be applied to the prior material balance and Darcy law equations that were initially used by Butler to develop Eq. 2-3. Azom and Srinivasan [12] used numerical methods to solve their developed equations and thus, their method goes under the category of semi analytical models. 


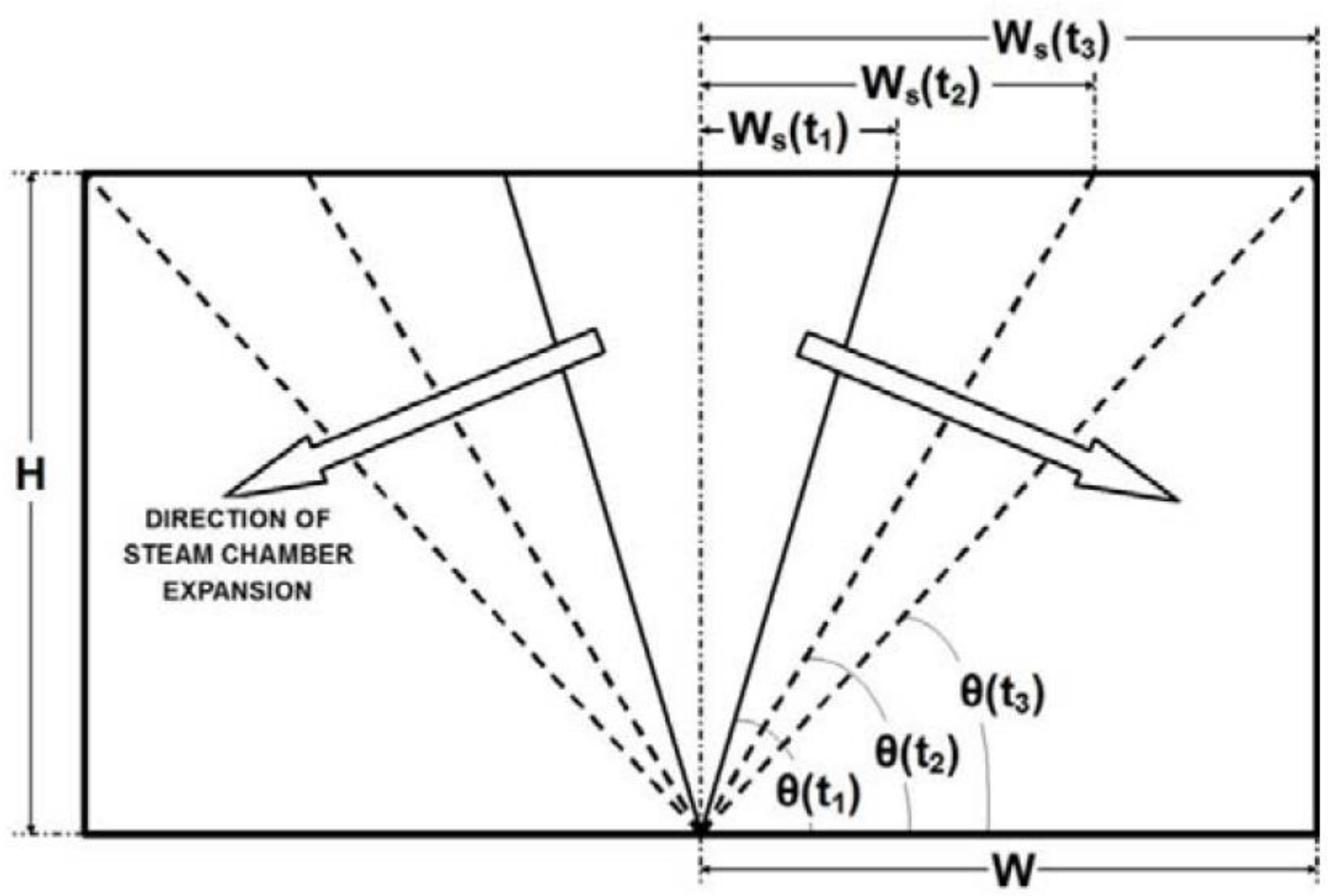

Figure 2-2: Schematic of an idealized SAGD chamber during horizontal growth (Courtesy of [12])

Kumar [13] studied the permeability heterogeneities in reservoirs that appear in the form of randomly formed shale lens (streaks) thorough the media. These lenses were generated based on a specific unique length and frequency using the Geostatistical Modeling Software (SGeMS). In the next step, Kumar [13] used s flow-based upscaling method (Durlofsky [14]) to assign each realization an equivalent vertical and horizontal permeability. Anisotropic models were created in CMG STARS using the realizations. The results were compared with the one calculated by analytical model (Azom et al. [12]) with the equivalent values of permeability. In lower shale volume percentage and shorter length correlation, the two methods showed close match but as either of these parameters increased the flow rate curves versus time tended to diverge in two models. That problem was related to the upscaling method they used that could not capture the impact of frequency and correlation length of shale barriers independently and the resultant permeability values were not accurate. The analytical model that was developed based on the Butler's model, overestimated the real flow rate because it could take into account the impact of shale lens into calculation. They improved their method by applying a more accurate upscaling 
method that used statistical averaging technique to take into account the behavior of multiphase fluid, and 3D structural nature of reservoir. This modification improved their results and the analytical models could be matched with numerical counterparts in larger range of shale streak correlation length and shale volume percentages.

In addition, the heterogeneities in their approach was treated as shale barriers that exist in the sand medium with a specific permeability value. In essence, permeability only can take two values, sand stone and shale permeability.

The spectral approach has been used to deal different types of problems. Gelhar and et al. [15] used this approach to treat time-variable problems. Freeze [16] and Bakr et al. [17] used the spectral-based technique in subsurface hydrology to solve the spatial variable problems. But no similar study was done on SAGD process.

All the authors improved analytical modeling of SAGD process by introducing the real-life aspects not included in the original Butler model. But none incorporated the heterogeneity of permeability and thermal diffusivity into the main equations. 


\section{Chapter 3. Methodology}

Butler's model [2] is based on the assumption that the medium is homogeneous. In this work, heterogeneity of the fluid and rock properties- permeability and thermal diffusivity- are integrated in the modeling of the SAGD process using a probability-based analysis. This technique is used to include local variations in the fluid and rock properties by assigning random perturbations to each property. Therefore, each property is decomposed into the expectation and perturbation parameters. The governing equations used to model SAGD process, namely heat diffusion equation and Darcy equation, are then modified to include perturbed properties. In the next step, Fourier-Stieltjes transform is applied to the developed equations to transform the cross-correlations of the perturbed parameters to term consisting of the mean and correlation of the properties. The upscaled equations are then developed by inputting the transformed terms into the original equations (Figure 3-1).

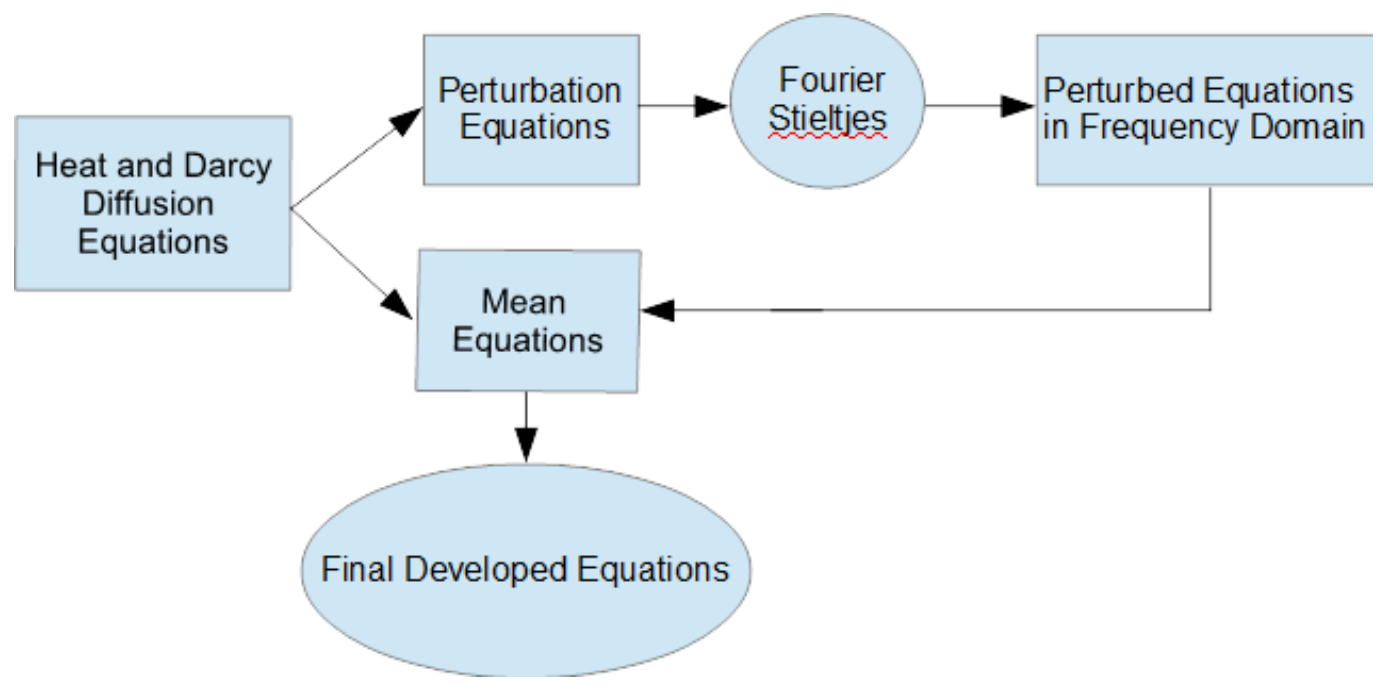

Figure 3-1: Flow Diagram of steps to obtain the new SAGD model

\subsection{Fourier-Stieltjes integral and spectral representation}

One of the key assumptions in developing the upscaled equations is that the process is secondorder stationary; in other words, 


$$
\begin{array}{r}
E[X(x)]=\mu R\left(x_{1}, x_{2}\right)=R\left(x-x_{2}\right)=R(\tau) ; \quad \tau \\
=x_{1}-x_{2} \quad \text { for any } x_{1}, x_{2}
\end{array}
$$

According to Gelhar [19], for a stationary process $X(t)$ with zero mean, the spectral representation of the process is

$$
X(x)=\int_{-\infty}^{\infty} e^{i \omega x} d Z(\omega)
$$

where $\omega$ is the angular frequency. In this equation, $\mathrm{Z}$ is a stochastic process with the following properties:

$$
\begin{gathered}
E[d Z(\omega)]=0 \\
{\left[d Z\left(\omega_{1}\right) d Z^{*}\left(\omega_{2}\right)\right]=0 ; \omega_{1} \neq \omega_{2}}
\end{gathered}
$$

If $\omega_{1}=\omega_{2}=\omega$ then:

$$
E\left[d Z\left(\omega_{1}\right) d Z^{*}(\omega)\right]=d \emptyset(\omega)=S(\omega) d \omega
$$

where $\emptyset(\omega)$ is the integrated spectrum, and $S(\omega)$ is the spectral density function, or the spectrum. For a zero-mean stationary stochastic process, let's take it $\mathrm{X}(\mathrm{t})$, the covariance function can be written as:

$$
R(\tau)=\int_{-\infty}^{\infty} e^{i \omega \tau} S(\omega) d \omega
$$

In this equation, the covariance function is the inverse Fourier-Stieltjes transform of the spectral density function. The corresponding spectrum function is:

$$
S(\omega)=\frac{1}{2 \pi} \int_{-\infty}^{\infty} e^{-i \omega t} R(\tau) d \omega
$$

\subsection{The Correlation of Parameters}

A review on the previous done researches shows that the correlation of permeability with thermal conductivity is more of interest than with thermal conductivity (Popov et al. [28], Mielke et al. [29]). Conductivity and diffusivity are related to each other as:

$$
\alpha=\frac{k}{\rho c_{\rho}}
$$


where $\mathrm{k}$ is conductivity $[\mathrm{W} /(\mathrm{m} . \mathrm{K})], \rho$ is density $[\mathrm{kg} / \mathrm{m} 3]$ and $\mathrm{c}_{\mathrm{p}}$ is specific heat capacity $[\mathrm{J} /(\mathrm{m} 3 \mathrm{~K})]$. Pore fluid type and pore structure of rocks are the main factors that affect conductivity of sedimentary rocks (Popov et al. [28]). There is no general relationship between thermal diffusivity and permeability. For a specific porous media and fluid types, relationships between thermal diffusivity and permeability can be obtained. Mielke et al. [29] used samples taken from Wairakei, cores of Huka Falls Formation (soft, lacustrine-deposited sediments), Waiora Formation (variably consolidated, medium hard, hydrothermally altered volcanic tuff) and Karapiti Rhyolite (hard, altered rhyolite lava and breccias) and measured their permeability and thermal conductivity values. Their results showed a very poor correlation between these two properties. However, the measurement showed there was a negative correlation between porosity and thermal conductivity.

The analysis used in this modeling is based on the mathematical representation of the logpermeability process as a random field. Permeability $(k)$ and thermal diffusivity $(\alpha)$ are assumed to have a log-linear relationship indicated by correlation parameter $c$ (Eq. 3-5). The effect of both positive and negative correlation will be studied. We define a new parameter $f=\ln k$ in our calculations instead of permeability (k).

$$
\ln k(x)=f(x)=\bar{f}(x)+f^{\prime}(x)=\bar{f}(x)+c * \alpha^{\prime}(x) \quad \text { Eq. 3-5 }
$$

where $k$ is perambility, $f$ is natural $\log$ permeability and $f^{\prime}$ and $\bar{f}$ are its perturbation and mean values. $\alpha$ is thermal diffusivity and similarly $\alpha^{\prime}$ and $\bar{\alpha}$ are mean and perturbation values. If these properties have positive correlation, $\mathrm{c}$ is positive and likewise if the correlation between them is negative, constant $\mathrm{c}$ will be negative. Permeability, viscosity, temperature and thermal diffusivity of reservoir are considered to be perturbed properties that can be decomposed into the mean (overbar variables) and small perturbations (prime-over variables) as follows:

$$
\begin{gathered}
\operatorname{Ln} \frac{1}{v}=\overline{m(x)}+m^{\prime}(x)=\operatorname{Ln}\left(\frac{1}{v_{0}}\right)+\beta\left(T-T_{0}\right)+\beta T^{\prime} \\
\alpha=\bar{\alpha}+\alpha^{\prime} \quad T=\bar{T}+T^{\prime} \\
\ln k(x)=f(x)=\bar{f}(x)+f^{\prime}(x)
\end{gathered}
$$


Where $T, k$ and $v$ are temperature, permeability and viscosity and $\beta$ is correlation coefficient between $m\left(\frac{1}{v}\right)$ and temperature $(T)$.

\subsection{Heat diffusion equation}

The one-dimensional thermal conduction can be written as:

$$
\frac{\partial T}{\partial t}=\alpha \frac{\partial^{2} T}{\partial x^{2}}
$$

If $T$ and $\alpha$ are Substituted from Eq. 3-6 as the sum of their mean and perturbation values in Eq. 37, it can be shown easily that Eq. 3-8 is achieved. If we take the mean value from both sides and implement the assumption that mean values of perturbations are zero, mean equation of heat transfer (Eq. 3-9) is resulted. Finally, the perturbation equation (Eq. 3-10) is achieved by subtracting mean equation (Eq. 3-9) from main equation (Eq. 3-8).

$$
\begin{gathered}
\frac{\partial \bar{T}}{\partial t}+\frac{\partial T^{\prime}}{\partial t}=\bar{\alpha} \frac{\partial^{2} \bar{T}}{\partial x^{2}}+\bar{\alpha} \frac{\partial^{2} T^{\prime}}{\partial x^{2}}+\alpha^{\prime} \frac{\partial^{2} T^{\prime}}{\partial x^{2}}+\alpha^{\prime} \frac{\partial^{2} \bar{T}}{\partial x^{2}} \\
\frac{\partial \bar{T}}{\partial t}=\bar{\alpha} \frac{\partial^{2} \bar{T}}{\partial x^{2}}+\overline{\alpha^{\prime} \frac{\partial^{2} T^{\prime}}{\partial x^{2}}} \\
\frac{\partial T^{\prime}}{\partial t}=\alpha^{\prime} \frac{\partial^{2} \bar{T}}{\partial x^{2}}+\bar{\alpha} \frac{\partial^{2} T^{\prime}}{\partial x^{2}}
\end{gathered}
$$

$\overline{\alpha^{\prime} \frac{\partial^{2} T \prime}{\partial x^{2}}}$ in Eq. 3-9 is the term that should be estimated. In the Butler's method, the heat diffusion is developed in moving coordinates for the simplicity it brings to the solution of equations. Heat transfer can be assumed to be steady state in the moving coordinates frame. Before jumping into transformation of coordinates to the moving coordinates, we prove a lemma that relates $\mathrm{dZ}$ transform (Gelhar [18]) of a variable in cartesian coordinates to moving coordinates.

Lemma: If $\mathrm{C}$ is a variable in a one-directional movement of a boundary with $\mathrm{x}$ and $\xi$ denoting the distance from the boundary in terms of a fixed and moving coordinate so that:

$$
\xi=x-U \times t ; \quad U=\text { Constant }
$$

Then: 


$$
\left.\frac{\partial\left(d Z_{c}\right)}{\partial t}\right|_{x}=\left.\frac{\partial\left(d Z_{c}\right)}{\partial t}\right|_{\xi}
$$

Proof:

$$
\begin{gathered}
c^{\prime}(x, t)=\int_{-\infty}^{\infty} e^{i\left(k_{i}, x_{i}\right)} d Z_{c}\left(k_{i}, t\right) \\
=\int_{-\infty}^{\infty} e^{i\left(k_{1}\left(\xi_{1}+U t\right)+k_{2} \xi_{2}+k_{3} \xi_{3}\right)} \cdot d Z_{c}\left(k_{i}, t\right) \\
\left.\rightarrow \frac{\partial c^{\prime}(x, t)}{\partial t}\right|_{\xi_{i}} \\
=\int_{-\infty}^{\infty}\left[i k_{1} U \times e^{i\left(k_{1}\left(\xi_{1}+U t\right)+k_{2} \xi_{2}+k_{3} \xi_{3}\right)} \cdot d Z_{c}\left(k_{i}, t\right)\right. \\
\left.+e^{i\left(k_{1}\left(\xi_{1}+U t\right)+k_{2} \xi_{2}+k_{3} \xi_{3}\right)} \frac{\partial\left(d Z_{c}\right)}{\partial t}\right]= \\
\int_{-\infty}^{\infty} e^{i\left(k_{1}\left(\xi_{1}+U t\right)+k_{2} \xi_{2}+k_{3} \xi_{3}\right)}\left(i k_{1} U \times d Z_{c}\left(k_{i}, t\right)+\frac{\partial\left(d Z_{c}\right)}{\partial t}\right)
\end{gathered}
$$

Eq. 3-11

According to Eq. 3-11, dZ equivalence of $\left.\frac{\partial c^{\prime}(x, t)}{\partial t}\right|_{\xi_{i}}$ is:

$$
i k_{1} U \times d Z_{c}\left(k_{i}, t\right)+\frac{\partial\left(d Z_{c}\right)}{\partial t}
$$

According to Butler [2], $\left(\frac{\partial \mathrm{c}}{\partial \mathrm{t}}\right)_{\mathrm{x}}$ can be written as:

$$
\left(\frac{d c}{d t}\right)_{\xi}-U\left(\frac{\partial c}{\partial x}\right)_{t}=\left(\frac{\partial c}{\partial t}\right)_{x}
$$

Applying Fourier-Stejles transform on Eq. 3-13 gives

$$
\left.\frac{\partial Z_{c}}{\partial t}\right|_{x}=i k_{1} U \times d Z_{c}\left(k_{i}, t\right)+\left.\frac{\partial\left(d Z_{c}\right)}{\partial t}\right|_{\xi}-U i k_{1} d Z_{c}\left(k_{i}, t\right)=\frac{\partial\left(Z_{c}\right)}{\partial t}
$$

By applying this lemma to the perturbation equation of thermal diffusion, it yields:

$$
\frac{\partial T^{\prime}}{\partial t}=\alpha^{\prime} \frac{\partial^{2} \bar{T}}{\partial x^{2}}+\bar{\alpha} \frac{\partial^{2} T^{\prime}}{\partial x^{2}}
$$




$$
\begin{aligned}
\left.\rightarrow \frac{\partial\left(d Z_{T}\right)}{\partial t}\right|_{\xi} & =d Z_{\alpha} H-\bar{\alpha} k^{2} d Z_{T} \\
\left.\rightarrow \frac{\partial\left(d Z_{T}\right)}{\partial t}\right|_{\xi}+\bar{\alpha} k^{2} d Z_{T} & =H d Z_{\alpha}
\end{aligned}
$$

where $H=\frac{\partial^{2} \bar{T}}{\partial x^{2}}$.

Assume a general first order equation:

$$
\frac{d\left(d Z_{T}\right)}{d t}+B(t) d Z_{T}(t)=A(t) d Z_{\alpha}
$$

According to Welty et al. [18], the solution of this equation is:

$$
d Z_{T}(t) \cong \frac{A(t)}{B(t)}\left(1-e^{-B t}\right) d Z_{\alpha}
$$

Therefore, the solution to Eq. 3-16 will be:

$$
d Z_{T}(t) \cong \frac{H(t)}{\bar{\alpha} k^{2}}\left(1-e^{-\bar{\alpha} k^{2} t}\right) d Z_{\alpha}
$$

$\overline{\alpha^{\prime} \frac{\partial^{2} T \prime}{\partial x^{2}}}$ in Eq. 3-9 can be decomposed to terms mean thermal diffusivity and temperature using the following equation,

$$
\begin{gathered}
d Z \text { of }\left(\frac{\partial^{2} T^{\prime}}{\partial x^{2}}\right)=-k^{2} d Z_{T} \\
\rightarrow \frac{\overline{\partial^{2} T^{\prime}}}{\partial x^{2}} \alpha^{\prime}=\int_{-\infty}^{\infty} S_{T \alpha}(k) d k=\int_{-\infty}^{\infty}-\frac{H(t)}{\bar{\alpha}}\left(1-e^{-\bar{\alpha} k^{2} t}\right) S_{\alpha \alpha} d k
\end{gathered}
$$

Replacing $\overline{\alpha^{\prime} \frac{\partial^{2} T^{\prime}}{\partial x^{2}}}$ in Eq. 3-9 with Eq. 3-19, the thermal diffusivity equation can be written as:

$$
\begin{array}{r}
\frac{\partial \bar{T}}{\partial t}=\bar{\alpha} \frac{\partial^{2} \bar{T}}{\partial x^{2}}+\overline{\alpha^{\prime} \frac{\partial^{2} T^{\prime}}{\partial x^{2}}} \\
\frac{\partial \bar{T}}{\partial t}=\bar{\alpha} \frac{\partial^{2} \bar{T}}{\partial x^{2}}-\int_{-\infty}^{\infty} \frac{H(t)}{\bar{\alpha}}\left(1-e^{-\bar{\alpha} k^{2} t}\right) S_{\alpha \alpha} d k
\end{array}
$$

This equation can be written as: 


$$
\frac{\partial \bar{T}}{\partial t}=\frac{\partial^{2} \bar{T}}{\partial x^{2}}\left(\bar{\alpha}-\int_{-\infty}^{\infty} \frac{1}{\bar{\alpha}}\left(1-e^{-\bar{\alpha} k^{2} t}\right) S_{\alpha \alpha} d k\right)
$$

Eq. 3-20 is in terms of location $\mathrm{x}$ and time t. In order to write it in moving coordinates, following equality relationship can be:

$$
\left(\frac{d T}{d t}\right)_{\xi}=\left(\frac{\partial T}{\partial x}\right)_{t} U+\left(\frac{\partial T}{\partial t}\right)_{x}
$$

Substituting Eq. 3-20 in Eq. 3-21, the resultant equation will be:

$$
\left(\frac{d \bar{T}}{d t}\right)_{\xi}-\left(\frac{\partial \bar{T}}{\partial t}\right)_{t} U=\frac{\partial^{2} \bar{T}}{\partial x^{2}}\left(\bar{\alpha}-\int_{-\infty}^{\infty} \frac{1}{\bar{\alpha}}\left(1-e^{-\bar{\alpha} k^{2} t}\right) S_{\alpha \alpha} d k\right)
$$

As the SAGD is a very slow process, due to the low velocity of boundary, the exponential term in the above equation can be omitted. This assumption has also been verified using the numerical simulations. After applying this assumption, the upscaled equation for heat transfer equation in Butler [2] (equation 2.36) can be obtained:

$$
\begin{array}{r}
\left(\frac{d \bar{T}}{d t}\right)_{\xi}-\left(\frac{\partial \bar{T}}{\partial t}\right)_{t} U=\frac{\partial^{2} \bar{T}}{\partial x^{2}}\left(\bar{\alpha}-\int_{-\infty}^{\infty} \frac{1}{\bar{\alpha}} S_{\alpha \alpha} d k\right) \\
\rightarrow \frac{\partial^{2} \bar{T}}{\partial x^{2}}\left(\bar{\alpha}-\int_{-\infty}^{\infty} \frac{1}{\bar{\alpha}} S_{\alpha \alpha} d k\right)+\left(\frac{\partial \bar{T}}{\partial t}\right)_{t} U=\left(\frac{d \bar{T}}{d t}\right)_{\xi} \\
\rightarrow \alpha_{e q}=\bar{\alpha}-\int_{-\infty}^{\infty} \frac{1}{\bar{\alpha}} S_{\alpha \alpha} d k
\end{array}
$$

If the integral $\int_{-\infty}^{\infty} \frac{1}{\bar{\alpha}} S_{\alpha \alpha} d k$ is positive, the resultant thermal diffusivity will be less than the mean value. This reduces the temperature in front of the moving boundary. The steady state solution of Eq. 3-22 is:

$$
\frac{T-T_{R}}{T_{S}-T_{R}}=e^{-\frac{U \xi}{\alpha_{e q}}}
$$

Eq. 3-23 indicates that perturbations on thermal diffusivity have a negative effect on the heat transfer in the reservoir. Subsequently less production is expected for the heterogeneous reservoir compared with homogeneous case in SAGD process. 
In the next step, three different types of perturbations are applied to thermal diffusivity and the resulting equivalent thermal diffusivity is determined. The perturbation types studied are described by harmonic, exponential, and hollow functions:

Harmonic Function

$$
\begin{gathered}
S_{a a}=\frac{1}{2} a^{2}\left[\delta\left(k+k_{0}\right)+\delta\left(k-k_{0}\right)\right] \\
\int_{-\infty}^{\infty} \frac{1}{\bar{\alpha}} S_{\alpha \alpha} d k=\frac{a^{2}}{\bar{\alpha}}>0 \Rightarrow \alpha_{e q}=\bar{\alpha}-\frac{a^{2}}{\bar{\alpha}}<\bar{\alpha}
\end{gathered}
$$

Exponential Function

$$
\begin{gathered}
S_{a a}=\frac{\lambda \sigma^{2}}{\pi\left(1+\lambda^{2} k^{2}\right)} \\
\int_{-\infty}^{\infty} \frac{1}{\bar{\alpha}} S_{\alpha \alpha} d k=\frac{\sigma^{2}}{\bar{\alpha}}>0 \Rightarrow \alpha_{e q}=\bar{\alpha}-\frac{\sigma^{2}}{\bar{\alpha}}<\bar{\alpha}
\end{gathered}
$$

Hollow Function

$$
\begin{gathered}
S_{a a}=\frac{2 l^{3} \sigma^{2} k^{2}}{\pi^{2}\left(1+l^{2} k^{2}\right)^{2}} \\
\int_{-\infty}^{\infty} \frac{1}{\bar{\alpha}} S_{\alpha \alpha} d k=\frac{\sigma^{2}}{\pi \bar{\alpha}}>0 \Rightarrow \alpha_{e q}=\bar{\alpha}-\frac{\sigma^{2}}{\pi \bar{\alpha}}<\bar{\alpha}
\end{gathered}
$$

Applying the mentioned perturbation types showed that the equivalent thermal diffusivity is less than the mean thermal diffusivity coefficient. In other words, if the perturbation is one of these functions, the temperature of the region ahead of boundary will be less than homogeneous reservoir and less drainage rate will be produced.

\subsection{Darcy Equation}

In this section, the fluid flow equation in porous media, which describes the flow of oil and steam in porous media in SAGD process and is based on the Darcy equation, is derived for the perturbed variables. Figure 3-1 depicts a vertical section view of the SAGD process along with an enlarged view of the boundary illustrating the differential directions and variables. The steam and reservoir 
temperatures are denoted by $\mathrm{T}_{\mathrm{S}}$ and $\mathrm{T}_{\mathrm{R}}$, respectively. At a distance $\xi$ form the interface, Darcy's equation can be written for a section with unity width measured into the paper.

$$
d q=\frac{k}{v} g \sin (\theta) d \xi
$$

Where $\Theta$ is the angle that the boundary makes with horizon (Figure 1-4).Using this equation, Eq. 3-24 the rate of drainage of oil, dq, within the element $d \xi$ (Figure 3-2) is described. If all the variables in this equation are written as the sum of their mean and perturbation values. Eq. 3-24 can be written as:

$$
\frac{\overline{d q}}{d \xi}+\frac{d q^{\prime}}{d \xi}=e^{\bar{f}+f^{\prime}} e^{\bar{m}+m \prime} g \sin (\theta)
$$

If $e^{m \prime}$ and $e^{f^{\prime}}$ are written in Fouriers Series, Eq. 3-26 is obtained:

$$
\begin{gathered}
\frac{\overline{d q}}{d \xi}+\frac{d q^{\prime}}{d \xi}=\frac{K_{L}}{v_{L}}\left(1+f^{\prime}+\frac{f^{\prime 2}}{2}+\frac{f^{\prime 2}}{2} m^{\prime}+\frac{m^{\prime 2}}{2}+\frac{m^{\prime 2}}{2} f^{\prime}+m^{\prime} f^{\prime}\right. \\
+\cdots .) \sin (\theta)
\end{gathered}
$$

Where $K_{L}=e^{\bar{f}}$ and $\frac{1}{v_{L}}=e^{\bar{m}}$. If mean values are taken for both sides of Eq. 3-26 and then small perturbation assumption is applied on the terms of the series to ignore the terms that are higher than $2^{\text {nd }}$ order, mean equation (Eq. 3-27) will be resulted. Perturbation equation (Eq. 3-28) can be obtained by subtracting the mean equation (Eq. 3-27) from main equation (Eq. 3-26) and ignoring the $2^{\text {nd }}$ order and higher order terms.

$$
\begin{gathered}
\frac{\overline{d q}}{d \xi}=\frac{K_{L}}{v_{L}}\left(1+\frac{\overline{f^{\prime 2}}}{2}+\frac{\overline{m^{\prime 2}}}{2}+\overline{m^{\prime} f^{\prime}}\right) \sin (\theta) \\
\frac{d q^{\prime}}{d \xi}=\frac{K_{L}}{v_{L}}\left(f^{\prime}+\beta T^{\prime}\right) \sin (\theta)
\end{gathered}
$$

Eq. 3-27 can be written as:

$$
\frac{\overline{d q}}{d \xi}=\frac{K_{L}}{v_{L}}\left(1+\frac{\overline{f^{\prime 2}}}{2}+\frac{\overline{m^{\prime 2}}}{2}+\overline{m^{\prime} f^{\prime}}\right) \sin (\theta)=\frac{K_{L}}{v_{L}} A \sin (\theta)
$$




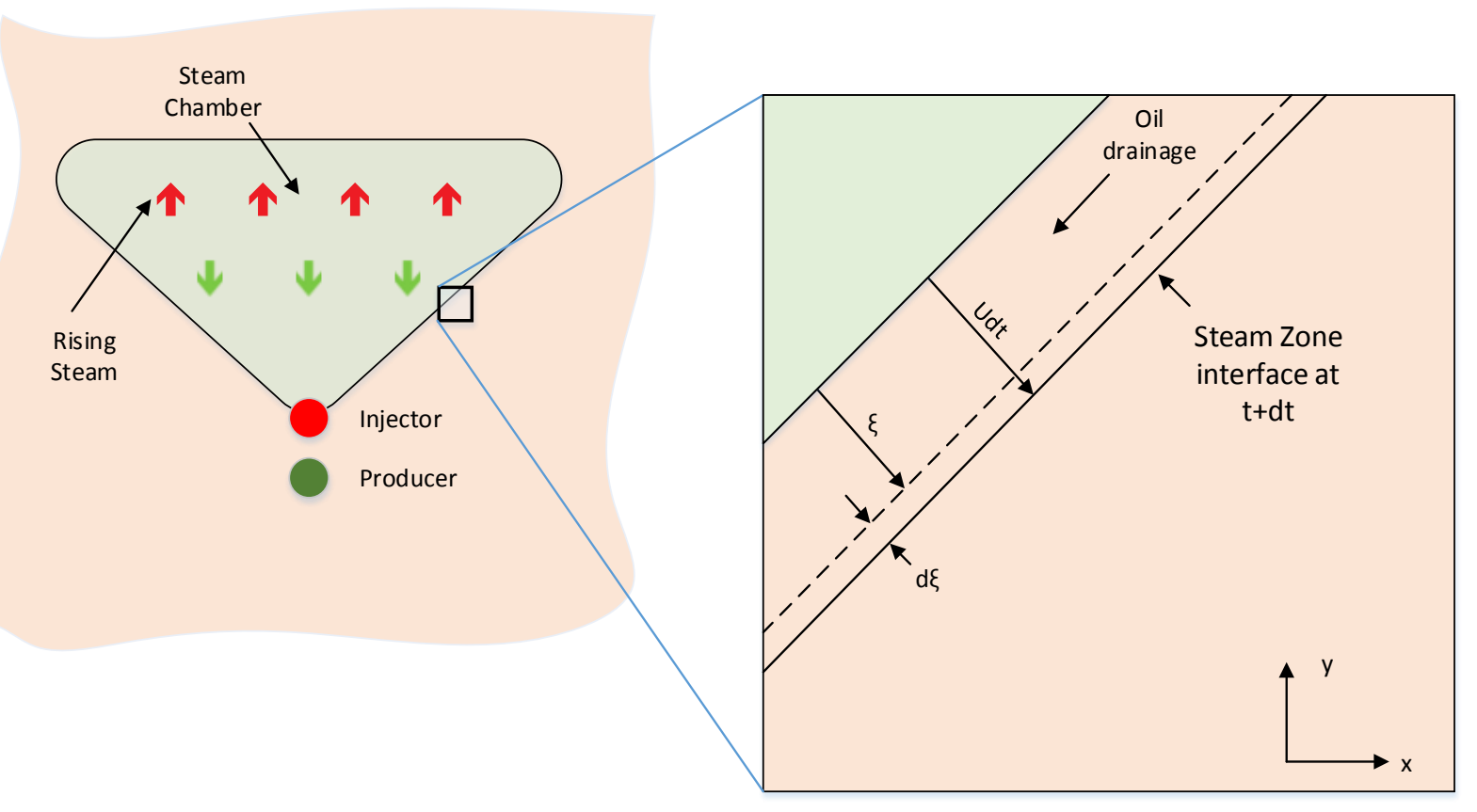

Figure 3-2: Cross Sectional View of SAGD along with the expanded view of the edge

$$
A=1+\frac{1}{2} \int_{-\infty}^{+\infty} S_{f f} d k+\frac{1}{2} \int_{-\infty}^{+\infty} S_{m m} d k+\int_{-\infty}^{+\infty} S_{m f} d k
$$

To develop Eq. 3-27, third order approximation in Taylor's series was used to simplify the equations, for a more detailed proof refer to Bakr et al. [17].

\subsection{Final Flow Equation}

1- As previously developed, $d Z_{T}$ can be calculated in terms of $d Z_{\alpha}$ through the heat equation:

$$
\left\{\begin{array}{c}
d Z_{T}=\frac{H}{\bar{\alpha} k^{2}} d Z_{\alpha} \\
\text { where }: \\
H=\frac{\partial^{2} \bar{T}}{\partial x^{2}} \\
\left.\bar{\alpha} \text { is mean value of thermal diffusivity (not } \alpha_{e q q}\right)
\end{array}\right.
$$

2- Reservoir and fluid properties in this study, are correlated as:

$$
\begin{gathered}
\ln k(x)=f(x)=\bar{f}(x)+f^{\prime}(x)=\bar{f}(x)+c * \alpha^{\prime}(x) \\
\operatorname{Ln} \frac{1}{v}=\overline{m(x)}+m^{\prime}(x)=\operatorname{Ln}\left(\frac{1}{v_{0}}\right)+\beta\left(T-T_{0}\right)
\end{gathered}
$$


Based on Eq. 3-29 and Eq. 3-30:

$$
\begin{gathered}
A=1+\frac{1}{2} \int_{-\infty}^{+\infty} S_{f f} d k+\frac{1}{2} \int_{-\infty}^{+\infty} S_{m m} d k+\int_{-\infty}^{+\infty} S_{m f} d k= \\
1+\frac{1}{2} \int_{-\infty}^{+\infty} c^{2} S_{\alpha \alpha} d k+\frac{1}{2} \int_{-\infty}^{+\infty} \frac{\beta^{2} H^{2}}{\bar{\alpha}^{2} k^{4}} S_{\alpha \alpha} d k+\int_{-\infty}^{+\infty} \frac{\beta c H}{\bar{\alpha} k^{2}} S_{\alpha \alpha} d k
\end{gathered}
$$

3-

$$
\frac{\left(\bar{T}-T_{R}\right)}{T_{S}-T_{R}}=e^{-B \xi} \Rightarrow d \xi=\frac{d \bar{T}}{-B\left(\bar{T}-T_{R}\right)}
$$

4-

$$
\begin{gathered}
\frac{d \bar{q}}{d \xi}=\frac{K_{L}}{v_{L}} A g \sin (\theta) \Rightarrow d \bar{q}=\frac{K_{L}}{v_{L}} A g \sin (\theta) d \xi \\
\quad \Rightarrow d \bar{q}=\frac{K_{L}}{v_{L}} A g \sin (\theta) \frac{d \bar{T}}{-B\left(\bar{T}-T_{R}\right)}
\end{gathered}
$$

5- calculation of $\frac{1}{v_{L}}$ :

$$
\begin{gathered}
\operatorname{Ln}\left(\frac{1}{v}\right)=\overline{m(x)}+m^{\prime}(x)=\operatorname{Ln}\left(\frac{1}{v_{R}}\right)+\beta\left(\bar{T}-T_{R}\right)+\beta T^{\prime}: \beta>0 \\
\Rightarrow \frac{1}{v_{L}}=e^{\overline{m(x)}}=\frac{1}{v_{R}} \times e^{\beta\left(\bar{T}-T_{R}\right)}
\end{gathered}
$$

6- If Eq. 3-49 is replaced in Eq. 3-38, then:

$$
d \bar{q}=\frac{K_{L}}{v_{R}} A g \sin (\theta) e^{\beta\left(\bar{T}-T_{R}\right)} \frac{d \bar{T}}{-B\left(\bar{T}-T_{R}\right)}
$$

7- H can be calculated thorough the solution of thermal diffusivity equation as:

$$
\begin{gathered}
H=\frac{\partial^{2} \bar{T}}{\partial x^{2}} \& \frac{\left(\bar{T}-T_{R}\right)}{T_{s}-T_{R}}=e^{-B \xi} \\
\Rightarrow H=B^{2}\left(T_{s}-T_{R}\right) e^{-B \xi}=B^{2}\left(T_{s}-T_{R}\right) \frac{\left(\bar{T}-T_{R}\right)}{T_{S}-T_{R}}=B^{2}\left(\bar{T}-T_{R}\right)
\end{gathered}
$$

8- Now, calculated $\mathrm{H}$ is replaced in Eq. 3-31 to give A as: 


$$
\begin{gathered}
A=1+\frac{1}{2} \int_{-\infty}^{+\infty} c^{2} S_{\alpha \alpha} d k+\frac{1}{2} \int_{-\infty}^{+\infty} \frac{\beta^{2} H^{2}}{\bar{\alpha}^{2} K^{4}} S_{\alpha \alpha} d k+\int_{-\infty}^{+\infty} \frac{2 \beta c H}{\bar{\alpha} k^{2}} S_{\alpha \alpha} d k \\
=1+\frac{1}{2} \int_{-\infty}^{+\infty} c^{2} S_{\alpha \alpha} d k+\frac{1}{2} \int_{-\infty}^{+\infty} \frac{\beta^{2} B^{4}\left(\bar{T}-T_{R}\right)^{2}}{\bar{\alpha}^{2} k^{4}} S_{\alpha \alpha} d k \\
+\int_{-\infty}^{+\infty} \frac{\beta c B^{2}\left(\bar{T}-T_{R}\right)}{\bar{\alpha} k^{2}} S_{\alpha \alpha} d k
\end{gathered}
$$

9- If the recent values obtained for A is replaced in Darcy Law, it gives:

$$
\begin{gathered}
d \bar{q}=\frac{K_{L}}{v_{R}} A g \sin (\theta) e^{\beta\left(\bar{T}-T_{R}\right)} \frac{d \bar{T}}{-B\left(\bar{T}-T_{R}\right)} \\
\Rightarrow d \bar{q}=\frac{K_{L} g \sin (\theta)}{-B v_{R}} \frac{e^{\beta\left(\bar{T}-T_{R}\right)} d \bar{T}}{\left(\bar{T}-T_{R}\right)} \times \\
\left(1+\frac{1}{2} \int_{-\infty}^{+\infty} c^{2} S_{\alpha \alpha} d k+\frac{1}{2} \int_{-\infty}^{+\infty} \frac{\beta^{2} B^{4}\left(\bar{T}-T_{R}\right)^{2}}{\bar{\alpha}^{2} k^{4}} S_{\alpha \alpha} d k+\int_{-\infty}^{+\infty} \frac{\beta c B^{2}\left(\bar{T}-T_{R}\right)}{\bar{\alpha} k^{2}} S_{\alpha \alpha} d k\right) \\
\Rightarrow d \bar{q}=\frac{K_{L} g \sin (\theta)}{-B v_{R}} \\
\times\left[\left(1+\frac{1}{2} \int_{-\infty}^{+\infty} c^{2} S_{\alpha \alpha} d k\right) \times \frac{e^{\beta\left(\bar{T}-T_{R}\right)} d \bar{T}}{\left(\bar{T}-T_{R}\right)}+\left(\frac{1}{2} \int_{-\infty}^{+\infty} \frac{\beta^{2} B^{4}}{\bar{\alpha}^{2} k^{4}} S_{\alpha \alpha} d k\right)\right. \\
\left.\times e^{\beta\left(\bar{T}-T_{R}\right)}\left(\bar{T}-T_{R}\right) d \bar{T}+\left(\int_{-\infty}^{+\infty} \frac{\beta c B^{2}}{\bar{\alpha} k^{2}} S_{\alpha \alpha} d k\right) \times e^{\beta\left(\bar{T}-T_{R}\right)} d \bar{T}\right]
\end{gathered}
$$

10- Integrate the flow rate, $\mathrm{q}$, and equation and simplify the solution. For the sake of convenience, the terms inside the parenthesis are named as VV, MM, NN as:

$$
\begin{aligned}
V & =\left(1+\frac{1}{2} \int_{-\infty}^{+\infty} c^{2} S_{\alpha \alpha} d k\right) \\
M M & =\left(\frac{1}{2} \int_{-\infty}^{+\infty} \frac{\beta^{2} B^{4}}{\bar{\alpha}^{2} k^{4}} S_{\alpha \alpha} d k\right) \\
N N & =\left(\int_{-\infty}^{+\infty} \frac{\beta c B^{2}}{\bar{\alpha} k^{2}} S_{\alpha \alpha} d k\right)
\end{aligned}
$$




$$
\begin{aligned}
\Rightarrow d \bar{q}=\frac{K_{L} g}{} & \sin (\theta) \\
- & B v_{R} \\
\times & {\left[V V \times \frac{e^{\beta\left(\bar{T}-T_{R}\right)} d \bar{T}}{\left(\bar{T}-T_{R}\right)}+M M \times e^{\beta\left(\bar{T}-T_{R}\right)}\left(\bar{T}-T_{R}\right) d \bar{T}+N N\right.} \\
& \left.\times e^{\beta\left(\bar{T}-T_{R}\right)} d \bar{T}\right]
\end{aligned}
$$

Integral form both sides, then:

$$
\begin{aligned}
\bar{q}=\frac{K_{L} g \sin (\theta)}{-B v_{R}} & \\
& \times\left[V V \times \int_{T_{S}}^{T_{R}+\epsilon} \frac{e^{\beta\left(\bar{T}-T_{R}\right)} d \bar{T}}{\left(\bar{T}-T_{R}\right)}+M M\right. \\
& \times \int_{T_{S}}^{T_{R}+\epsilon} e^{\beta\left(\bar{T}-T_{R}\right)}\left(\bar{T}-T_{R}\right) d \bar{T}+N N \\
& \left.\times \int_{T_{S}}^{T_{R}+\epsilon} e^{\beta\left(\bar{T}-T_{R}\right)} d \bar{T}\right]
\end{aligned}
$$

Eq. 3-38 offers the flow rate produced along the reservoir with a depth of 1 meter when Steam chamber is run through the reservoir. Note that the upper limit of integral on the right side of the developed equation cannot be temperature of reservoir in the normal condition, otherwise, flow rate will be infinity. In practice, it never reaches the temperature of reservoir along $\xi$ direction. To evade this problem, an infinitesimal value, $\epsilon$, is added to upper limit of integral.

$$
\begin{aligned}
\bar{q}= & \frac{K_{L} g \sin (\theta)}{\cos \theta\left(\frac{\partial y}{\partial t}\right)_{x}} \\
+\frac{\bar{\alpha}-\frac{a^{2}}{\bar{\alpha}}}{v_{R}} & \\
& \times\left[V V \times \int_{T_{S}}^{T_{R}+\epsilon} \frac{e^{\beta\left(\bar{T}-T_{R}\right)} d \bar{T}}{\left(\bar{T}-T_{R}\right)}+M M \times \int_{T_{S}}^{T_{R}+\epsilon} e^{\beta\left(\bar{T}-T_{R}\right)}\left(\bar{T}-T_{R}\right) d \bar{T}+N N\right. \\
& \left.\times \int_{T_{S}}^{T_{R}+\epsilon} e^{\beta\left(\bar{T}-T_{R}\right)} d \bar{T}\right]
\end{aligned}
$$




$$
\begin{gathered}
\Rightarrow \bar{q}=\left(\bar{\alpha}-\frac{a^{2}}{\bar{\alpha}}\right) \frac{K_{L} g \tan (\theta)}{v_{R}} * \frac{1}{\left(\frac{\partial y}{\partial t}\right)_{x}} \\
\times\left[V V \times \int_{T_{S}}^{T_{R}+\epsilon} \frac{e^{\beta\left(\bar{T}-T_{R}\right)} d \bar{T}}{\left(\bar{T}-T_{R}\right)}+M M \times \int_{T_{S}}^{T_{R}+\epsilon} e^{\beta\left(\bar{T}-T_{R}\right)}\left(\bar{T}-T_{R}\right) d \bar{T}+N N\right. \\
\left.\times \int_{T_{S}}^{T_{R}+\epsilon} e^{\beta\left(\bar{T}-T_{R}\right)} d \bar{T}\right] \\
\Rightarrow \bar{q}=\left(\bar{\alpha}-\frac{a^{2}}{\bar{\alpha}}\right)^{\frac{K_{L} g\left(\frac{\partial y}{\partial x}\right)_{t}}{v_{R}} \frac{1}{\left(\frac{\partial y}{\partial t}\right)_{x}}} \\
\times \int_{T^{\prime}} \times \int_{T_{S}}^{T_{R}+\epsilon} \frac{e^{\beta\left(\bar{T}-T_{R}\right)} d \bar{T}}{\left(\bar{T}-T_{R}\right)}+M M \\
\times \int_{T_{S}}^{T_{R}+\epsilon} e^{\beta\left(\bar{T}-T_{R}\right)}\left(\bar{T}-T_{R}\right) d \bar{T}+N N \\
\left.\times \int_{T_{S}}^{T_{R}+\epsilon} e^{\beta\left(\bar{T}-T_{R}\right)} d \bar{T}\right]
\end{gathered}
$$

Material balance can be written as:

$$
\left(\frac{\partial \bar{q}}{\partial x}\right)_{t}=\bar{\phi} \Delta S_{o}\left(\frac{\partial y}{\partial t}\right)_{x} \Rightarrow\left(\frac{\partial y}{\partial t}\right)_{x}=\frac{1}{\bar{\phi} \Delta S_{o}}\left(\frac{\partial \bar{q}}{\partial x}\right)_{t}
$$

If $\left(\frac{\partial \bar{q}}{\partial x}\right)_{t}$ is substituted in the flow rate equation (Eq. 3-38). 


$$
\begin{aligned}
& \Rightarrow \bar{q}=\left(\bar{\alpha}-\frac{a^{2}}{\bar{\alpha}}\right) \frac{K_{L} g}{v_{R}} * \bar{\phi} \Delta S_{o} *\left(\frac{\partial y}{\partial q}\right)_{t} \\
& \times\left[V V \times \int_{T_{S}}^{T_{R}+\epsilon} \frac{e^{\beta\left(\bar{T}-T_{R}\right)} d \bar{T}}{\left(\bar{T}-T_{R}\right)}+M M\right. \\
& \times \int_{T_{S}}^{T_{R}+\epsilon} e^{\beta\left(\bar{T}-T_{R}\right)}\left(\bar{T}-T_{R}\right) d \bar{T}+N N \\
&\left.\times \int_{T_{S}}^{T_{R}+\epsilon} e^{\beta\left(\bar{T}-T_{R}\right)} d \bar{T}\right]
\end{aligned}
$$

If differential variables are separated and both sides are integrated, then:

$$
\begin{aligned}
& \quad \bar{q}=\sqrt{-2\left(\bar{\alpha}-\frac{a^{2}}{\bar{\alpha}}\right) \frac{K_{L} g}{v_{R}} * \bar{\phi} \Delta S_{o} * h * A A} \\
& \text { Where } \quad A A=\left[V V \times \int_{T_{S}}^{T_{R}+\epsilon} \frac{e^{\beta\left(\bar{T}-T_{R}\right)} d \bar{T}}{\left(\bar{T}-T_{R}\right)}+M M \times\right. \\
& \left.\int_{T_{S}}^{T_{R}+\epsilon} e^{\beta\left(\bar{T}-T_{R}\right)}\left(\bar{T}-T_{R}\right) d \bar{T}+N N \times \int_{T_{S}}^{T_{R}+\epsilon} e^{\beta\left(\bar{T}-T_{R}\right)} d \bar{T}\right]
\end{aligned}
$$

After solving the integrals in Eq. 3-38, AA is calculated as:

$$
\begin{aligned}
A A=[V V \times[ & \left.E i(\beta \epsilon)-E i\left(\beta *\left(T_{S}-T_{R}\right)\right)\right]+M M \\
& \times\left[\frac{e^{\beta \epsilon}(\beta \epsilon-1)}{\beta^{2}}-\frac{\left(e^{\beta\left(T_{S}-T_{R}\right)}\left(\beta\left(T_{S}-T_{R}\right)-1\right)\right.}{\beta^{2}}\right] \\
& \left.+N N \times \frac{1}{\beta}\left(e^{\beta \epsilon}-e^{\beta\left(T_{S}-T_{R}\right)}\right)\right]
\end{aligned}
$$

Where $E_{i}$ is the exponential integral:

$$
\operatorname{Ei}\left(x_{0}\right)=\int_{1}^{\infty} \frac{e^{-x_{0} t} d t}{t}=\int_{x_{0}}^{\infty} \frac{e^{-u} d u}{u}
$$

VV, MM and NN values are as defined by Eq. 3-21: 


\subsection{Position of Boundary}

The horizontal velocity of the interface is given by

$$
\left(\frac{\partial x}{\partial t}\right)_{y}=-\frac{\left(\frac{\partial y}{\partial t}\right)_{x}}{\left(\frac{\partial y}{\partial x}\right)_{t}}
$$

Eq. 3-41

If right side of Eq. 3-41 is replaced with second raw in Eq. 3-36, then:

$$
\left(\frac{\partial x}{\partial t}\right)_{y}=-\frac{\left(\bar{\alpha}-\frac{a^{2}}{\bar{\alpha}}\right)}{q} \frac{K_{L} g}{v_{R}}
$$

If $\mathrm{q}$ from Eq. 3-39 is replaced in Eq. 3-41, then:

$$
\begin{aligned}
\left(\frac{\partial x}{\partial t}\right)_{y} & =\frac{\left(\bar{\alpha}-\frac{a^{2}}{\bar{\alpha}}\right)}{\sqrt{-2\left(\bar{\alpha}-\frac{a^{2}}{\bar{\alpha}}\right) \frac{K_{L} g}{v_{R}} * \bar{\phi} \Delta S_{o} *(h-y) * A A}} \frac{K_{L} g}{v_{R}} \\
& \Rightarrow x=t * \sqrt{-\frac{\left(\bar{\alpha}-\frac{a^{2}}{\bar{\alpha}}\right) \frac{K_{L} g}{v_{R}}}{2 * \bar{\phi} \Delta S_{o} *(h-y) * A A}}
\end{aligned}
$$

Eq. 3-49 can be rearranged to give $y$ in terms of $x$ and $t$ :

$$
y=h+\frac{\left(\bar{\alpha}-\frac{a^{2}}{\bar{\alpha}}\right) \frac{K_{L} g}{v_{R}}}{2 * \bar{\phi} \Delta S_{o} * A A}\left(\frac{t}{x}\right)^{2}
$$

Using the dimensionless form of variables $\mathrm{x}, \mathrm{y}$ and t, Eq. 3-49 can be written as:

$$
\begin{gathered}
Y=1-1 / 2\left(\frac{t^{\prime}}{X}\right)^{2} \\
Y=\frac{y}{h}
\end{gathered}
$$

Eq. 3-47 


$$
\begin{gathered}
X=\frac{x}{h} \\
t^{\prime}=\frac{t}{h} \sqrt{\left(-\frac{\left(\bar{\alpha}-\frac{a^{2}}{\bar{\alpha}}\right) \frac{K_{L} g}{v_{R}}}{\bar{\phi} \Delta S_{o} * A A}\right)}
\end{gathered}
$$

Eq. 3-49 


\section{Chapter 4. Verification of Upscaled Thermal Equation}

In previous section, an equivalent diffusivity coefficient has been developed based on FourierStieltjes transform for three types of thermal diffusivity. In this section; a verification will be considered to test the results. This verification generates inputs based on their distributions and will used arithmetic averaging to estimate the mean values of temperature that is a key variable in SAGD process. The results will be compared with the one obtained theoretically in previous sections.

Numerical verification in this step of research will be a useful tool to resolve the two concerns that have risen so far. First, the method that Gelhar [18] suggested in his works was based on FourierStieltjes transform but he did not verify his model with any reliable method. There are some questions in this method that we could not find a reasonable answer for them. For instance, as raised in previous reports, taking the gradient of mean concentration in diffusivity equation as a constant value while moving from the space - time frame to spectrum frame lacks a reasonable justification. If verification is passed successfully, we can make sure our solution was correct.

Secondly, a numerical verification helps us have a better understanding of real-life data, the concept of correlation and spectrum. Inputs are produced based on their correlation. Harmonic correlation function will be used to generate a sufficient number of realizations. This type of verification is unique and has not been included in any previous similar studies.

Stochastic analysis is generally applied on two processes, stochastic or time series processes vs spatial variability or random fields. These two categories have been explained and addressed with details in Gelhar [2].

In this research, thermal diffusivity and permeability are assigned with perturbations and are given a specific distribution throughout the reservoir, making it act as a random field. However; the reservoir is exposed to vary over time as a deterministic variable. This type of stochastic modeling that combines the spacious randomness with time varying characteristic, includes the transient solution of the model. Gelhar [19] did not mention this type of analysis but partially mentioned it in [18]. 
Three common functions were evaluated in previous section and their equivalent thermal diffusivities were calculated based on the spectrum-based analysis: harmonic, exponential and hole type function. Let us list the correlation functions and their spectrums here [2]:

Harmonic Function:

$$
\begin{gathered}
S_{a a}=\frac{1}{2} a^{2}\left[\delta\left(k+k_{0}\right)+\delta\left(k-k_{0}\right)\right] \\
R(\tau)=\frac{a^{2}}{2} \cos \left(\omega_{0} \tau\right), \quad \tau=t_{2}-t_{1}
\end{gathered}
$$

Exponential Function:

$$
\begin{gathered}
S_{a a}=\frac{\sigma^{2} \lambda}{\pi\left(1+\lambda^{2} k^{2}\right)} \\
R(\tau)=\sigma^{2} e^{\frac{-|\tau|}{\lambda}}
\end{gathered}
$$

Hollow Function

$$
\begin{gathered}
S_{a a}=\frac{2 l^{3} \sigma^{2} k^{2}}{\pi^{2}\left(1+l^{2} k^{2}\right)^{2}} \\
R(\tau)=\sigma^{2}(1-|\tau| / l) e^{-|\tau| / l}
\end{gathered}
$$

All above spectral functions have used notation $\mathrm{k}$ as the frequency in one-dimensional space. In case of stochastic processes with times series, the notation $\omega$ is used. We need distribution functions to generate realizations for this case. But only the harmonic distribution function was available in [19] and we could not find the distribution functions for the other two types of correlation functions.

We apply Monte Carlo method to verify our results, realizations are generated based on the harmonic distributions. Because the process is known only for harmonic function, we do the verification based on this distribution. According to Gelhar [19], harmonic process/field can be written as:

$$
\alpha^{\prime}(x)=\bar{\alpha} a_{\alpha} \cos \left(\omega_{0} x+\phi\right) \quad 0<a_{\alpha}<1
$$


50 and 100 field samples of thermal diffusivity are chosen based on this distribution. The parameters of diffusivity perturbations are to be chosen as $a_{\alpha}=0.7$ and $\omega=\frac{\pi}{4}$. The thermal equation (Eq. 4-1) is solved for each of these samples. Temperature curves of 50 realizations are shown in Figure 4-1. In averaging technique, the mean value of a large enough number of realizations is introduced as the most probable ultimate solution. The averaged-based temperature curve is shown in Figure 4-2 in black color.

$$
X\left(\frac{\partial T}{\partial t}\right)_{x}=\left(\frac{d T}{d t}\right)_{\xi}-U\left(\frac{\partial T}{\partial x}\right)_{t}
$$

To verify our heterogeneous model, the developed equation for heterogeneous reservoir in this research that is Eq. 4-2 is solved numerically. The green curve in Figure 4-2 represents the result obtained with theoretical analysis in which mean values of properties are used in the new modified equation (Eq. 4-2) rather than the perturbed variables themselves.

$$
\left(\frac{d \bar{T}}{d t}\right)_{\xi}-\left(\frac{\partial \bar{T}}{\partial \xi}\right)_{t} U=\frac{\partial^{2} \bar{T}}{\partial \xi^{2}}\left(\bar{\alpha}-\int_{-\infty}^{\infty} \frac{1}{\bar{\alpha}}\left(1-e^{-\bar{\alpha} k^{2} t}\right) S_{\alpha \alpha} d k\right)
$$

Moreover, to illustrate the effects of perturbations on temperature distribution, the exact solution of thermal equation modeled by Butler, is drawn along with the previous two curves in Figure 4-2. As seen, perturbations of diffusivity reduce the temperature in the reservoir and cause a declining effect on the oil recovery. The reduced temperature in the reservoir inhibits the main purpose of situ processes that is reducing the viscosity of the heavy oil or bitumen. In addition, in Figure 4-2 the closeness of two curves obtained by upscaled model and average-based models verifies the accuracy of our developed model. The verification results in Figure 4-1 and Figure 4-2 are obtained by a 50 realizations test. However, to get more accuracy in comparison, more realizations can be used by average-based model. The same procedure is repeated for a set of 100 heterogeneous realizations all obtained by solving Eq. 4-1. The relevant curves are shown by Figure 4-3 and Figure 4-4. The test indicates that with 100 realizations, the oscillating average-based curve is getting closer to the upscaled model that is constituent with the basic concept of averaging in statistics. 


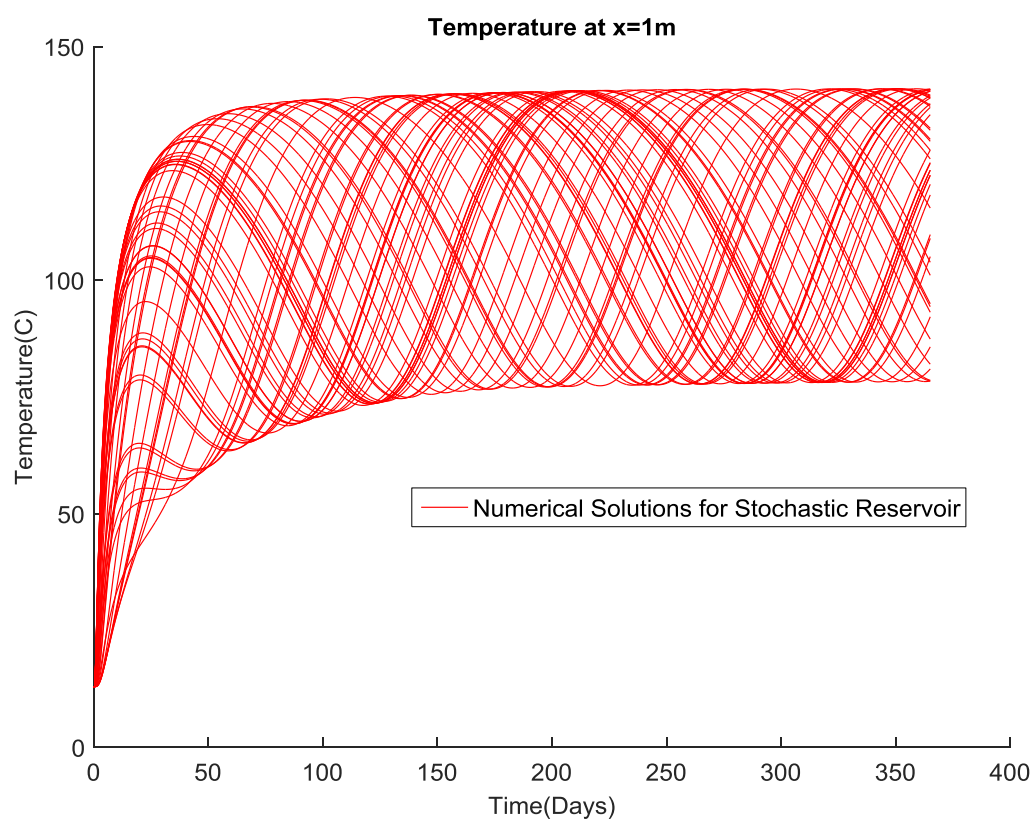

Figure 4-1: Temperature curves for 50 realizations at a location $1 \mathrm{~m}$ ahead of boundary for

$$
a_{\alpha}=0.7 \text { and } \omega=\frac{\pi}{4}
$$

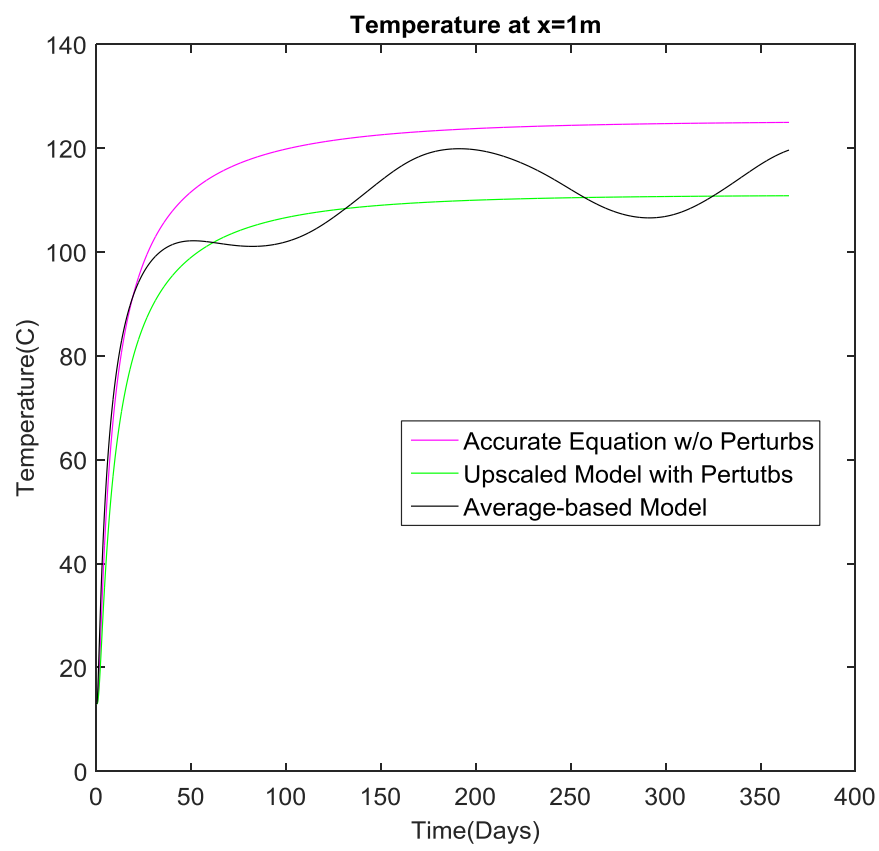

Figure 4-2: Comparison of developed upscaled model with Butler's model and average-based model of 50 realizations for $a_{\alpha}=0.7$ and $\omega=\frac{\pi}{4}$. 


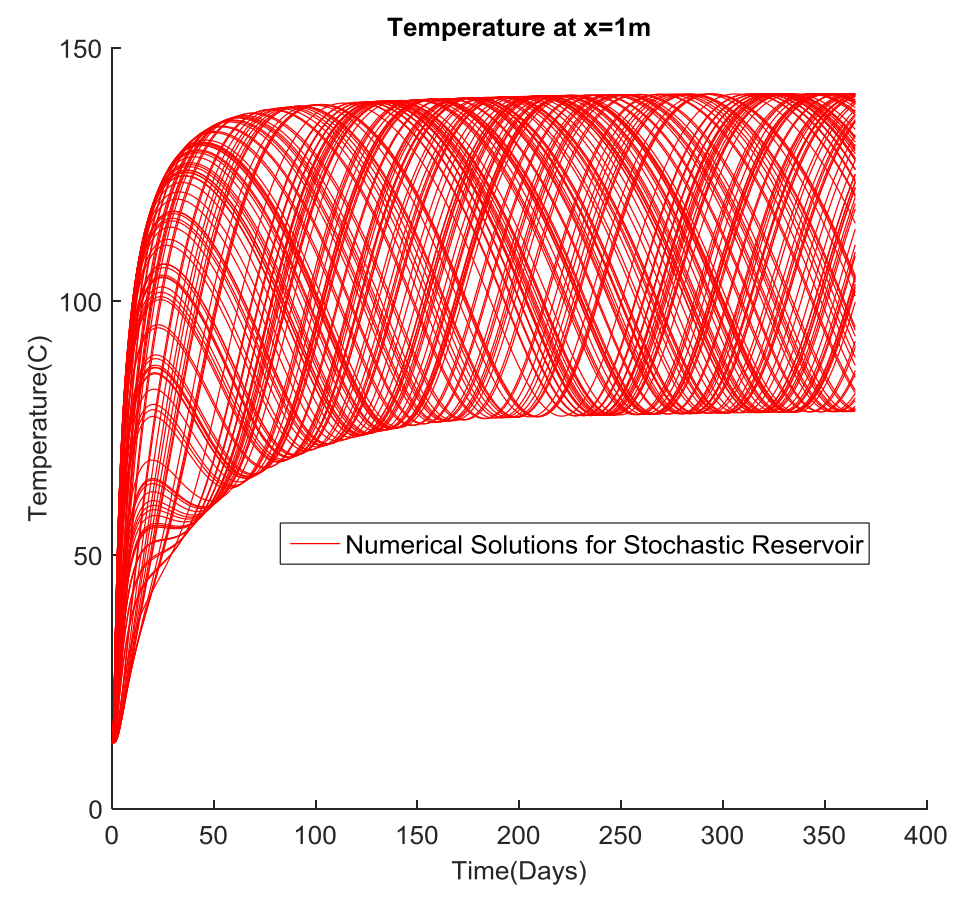

Figure 4-3: Temperature curves for 100 realizations at a location $1 \mathrm{~m}$ ahead of boundary for

$$
a_{\alpha}=0.7 \text { and } \omega=\frac{\pi}{4} \text {. }
$$

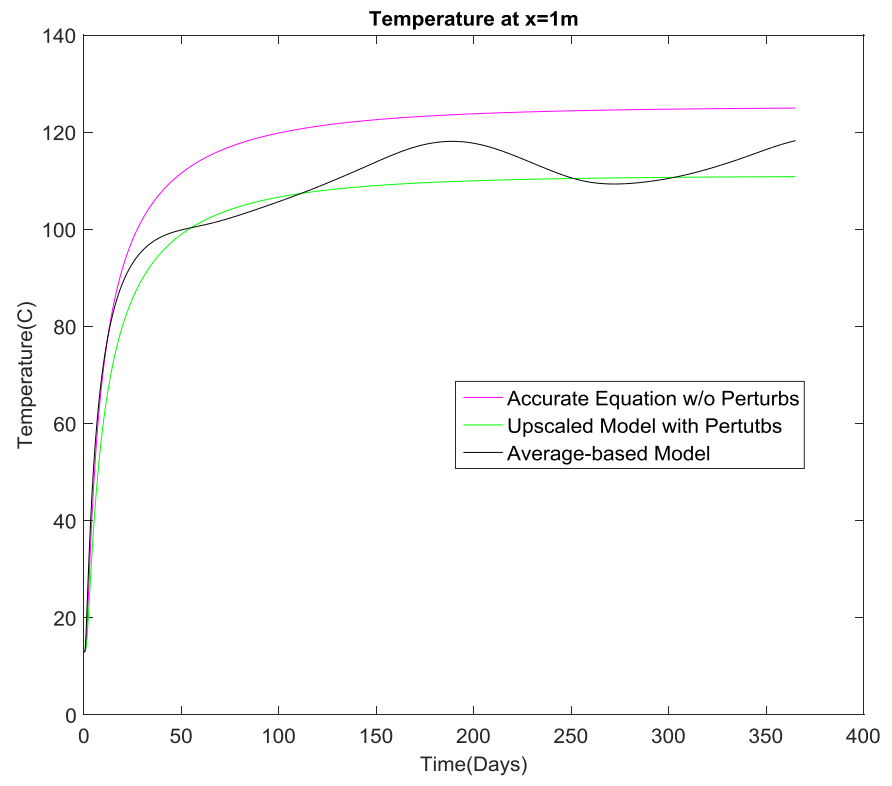

Figure 4-4: Comparison of developed upscaled model with Butler's model and average-based model of 100 realizations for $a_{\alpha}=0.7$ and $\omega=\frac{\pi}{4}$. 
In an additional test, angular frequency of harmonic functions $(\omega)$ used in our synthetic distributions was subjected to change. The temperature curves values of $\omega=$ $\frac{\pi}{2}, \pi, \frac{3 \pi}{2}$ and $2 \pi$ were obtained by applying the same averaging technique used for $\omega=\frac{\pi}{4}$. Figure 4-5, Figure 4-6, Figure 4-7 and Figure 4-8 depict the relevant temperature curves for different angular velocities. As seen all scenarios verify the accuracy of our developed upscaled model.

To do more validation, another test can also be done. So far, parameter $a_{\alpha}$ in all models has been assigned to be 0.7 . To observe its effect on the temperature curves, the test is repeated for $a_{\alpha}=$ 0.6, 0.8 and 0.9. The results are depicted in Figure 4-9, Figure 4-10 and Figure 4-11 show that the curve obtained by upscaled model deviates from average-based curve as the variation of diffusivity increases. This observation can be justified by the limitation imposed by the approximation we used whole developing our upscaled model. To get the final solution of the differential equation, Tylor's series approximation was used to solve the equation that is valid for reasonably small enough values of $\alpha^{\prime}$. In addition, 1st order approximation of perturbation in developing mean equations may be the other reason for this error. The results of simulations show that at $a_{\alpha} \leq 60$, the upscaled model offers acceptable accuracy. Accordingly, we will keep $a_{\alpha}$ within this range for the next section tests. 


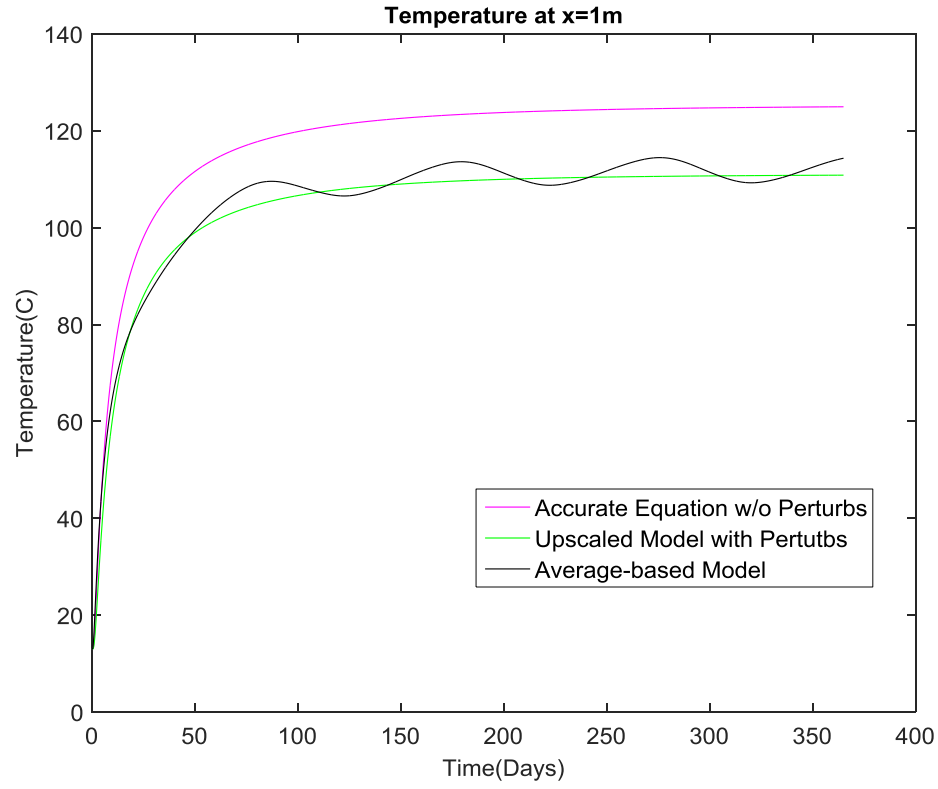

Figure 4-5: Comparison of developed upscaled model with Butler's model and average-based model of 100 realizations for $a_{\alpha}=0.7$ and $\omega=\frac{\pi}{2}$.

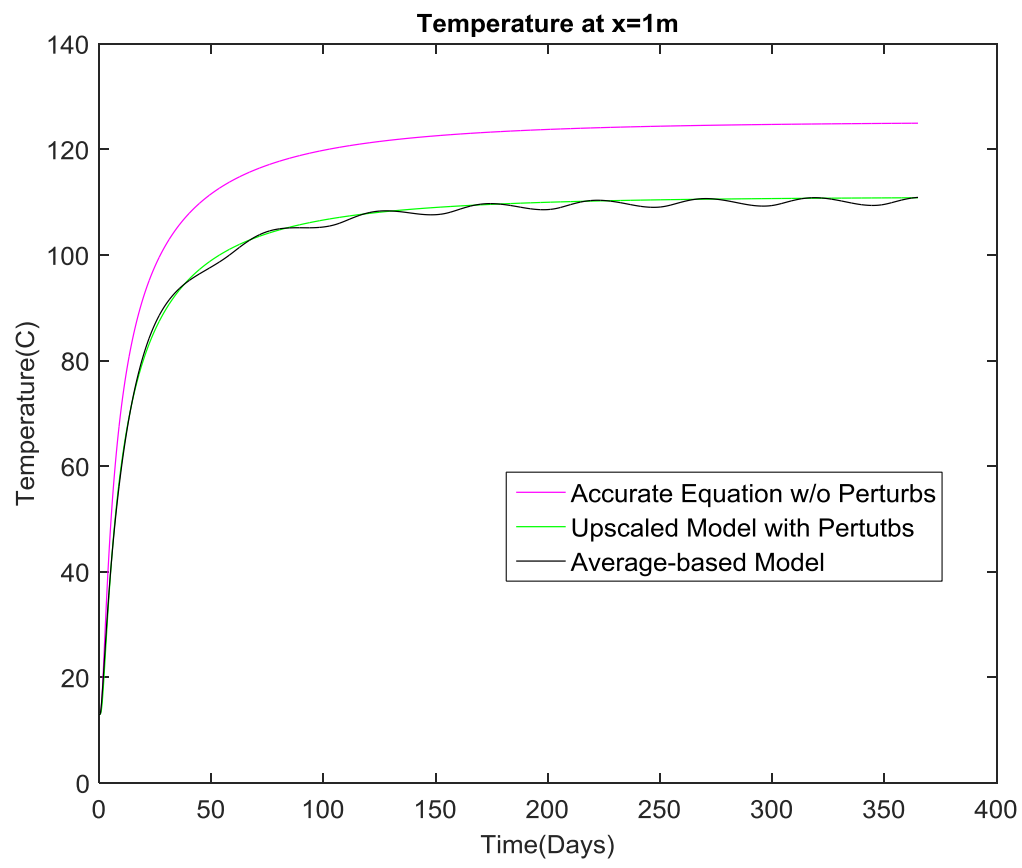

Figure 4-6: Comparison of developed upscaled model with Butler's model and average-based model of 100 realizations for $a_{\alpha}=0.7$ and $\omega=\pi$. 


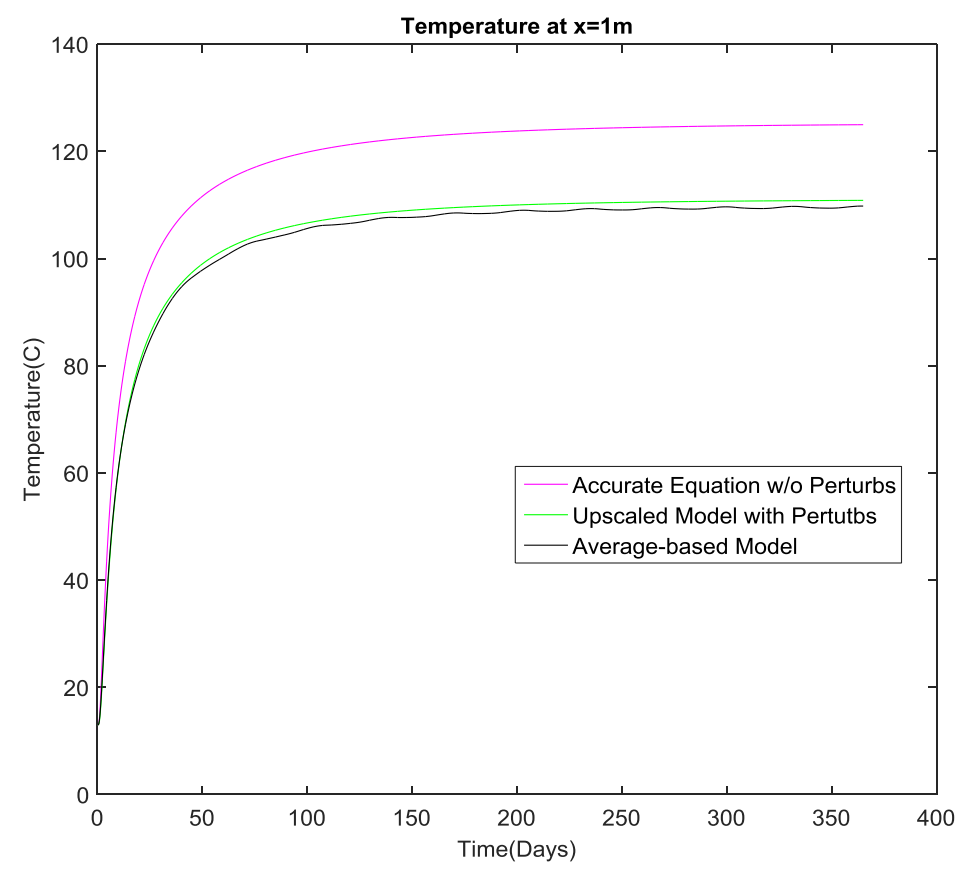

Figure 4-7: Comparison of developed upscaled model with Butler's model and average-based model of 100 realizations for $a_{\alpha}=0.7$ and $\omega=\frac{3 \pi}{2}$.

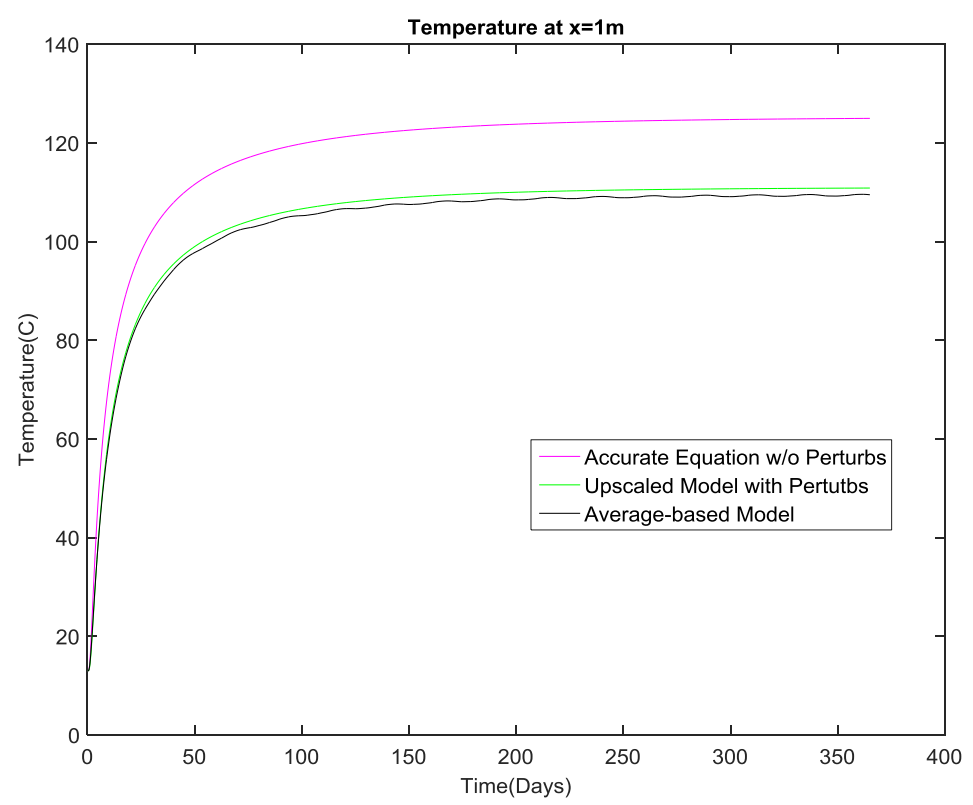

Figure 4-8: Comparison of developed upscaled model with Butler's model and average-based model of 100 realizations for $a_{\alpha}=0.7$ and $\omega=2 \pi$. 


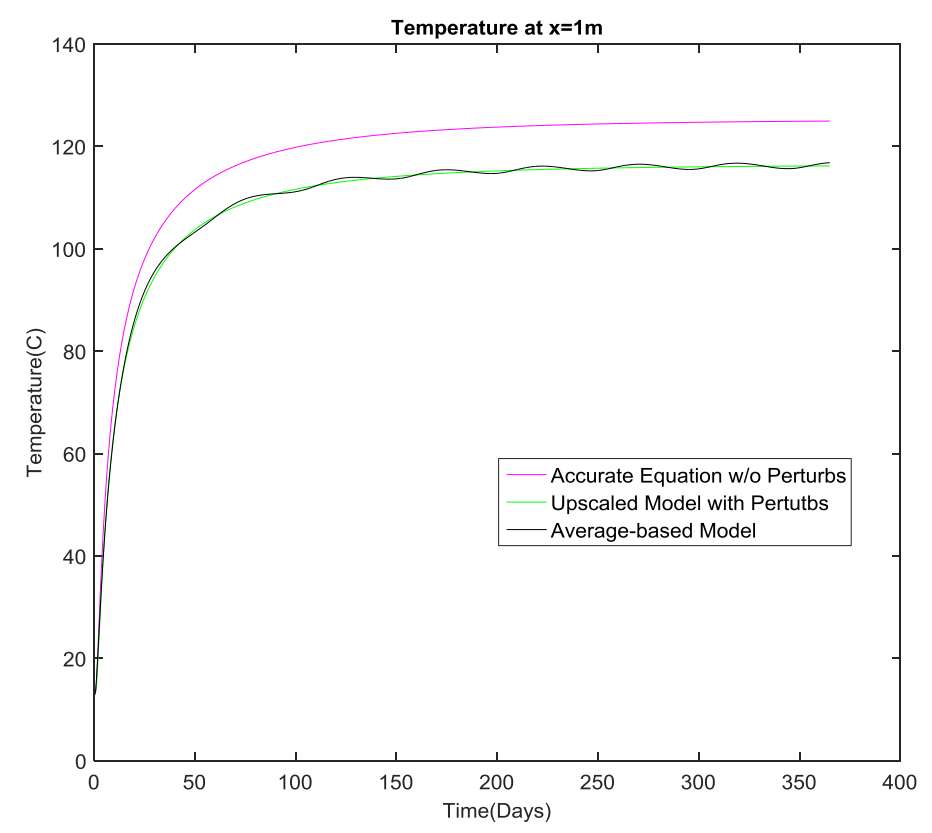

Figure 4-9: Comparison of developed upscaled model with Butler's model and average-based model of 100 realizations for $a_{\alpha}=0.6$ and $\omega=\pi$.

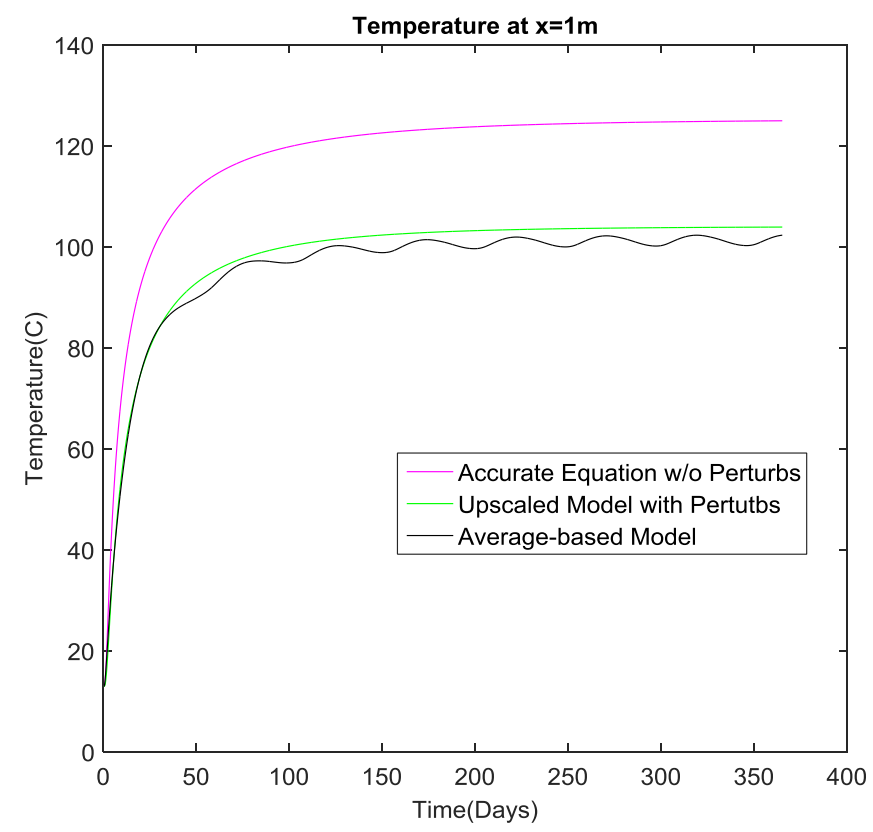

Figure 4-10: Comparison of developed upscaled model with Butler's model and average-based model of 100 realizations for $a_{\alpha}=0.8$ and $\omega=\pi$. 


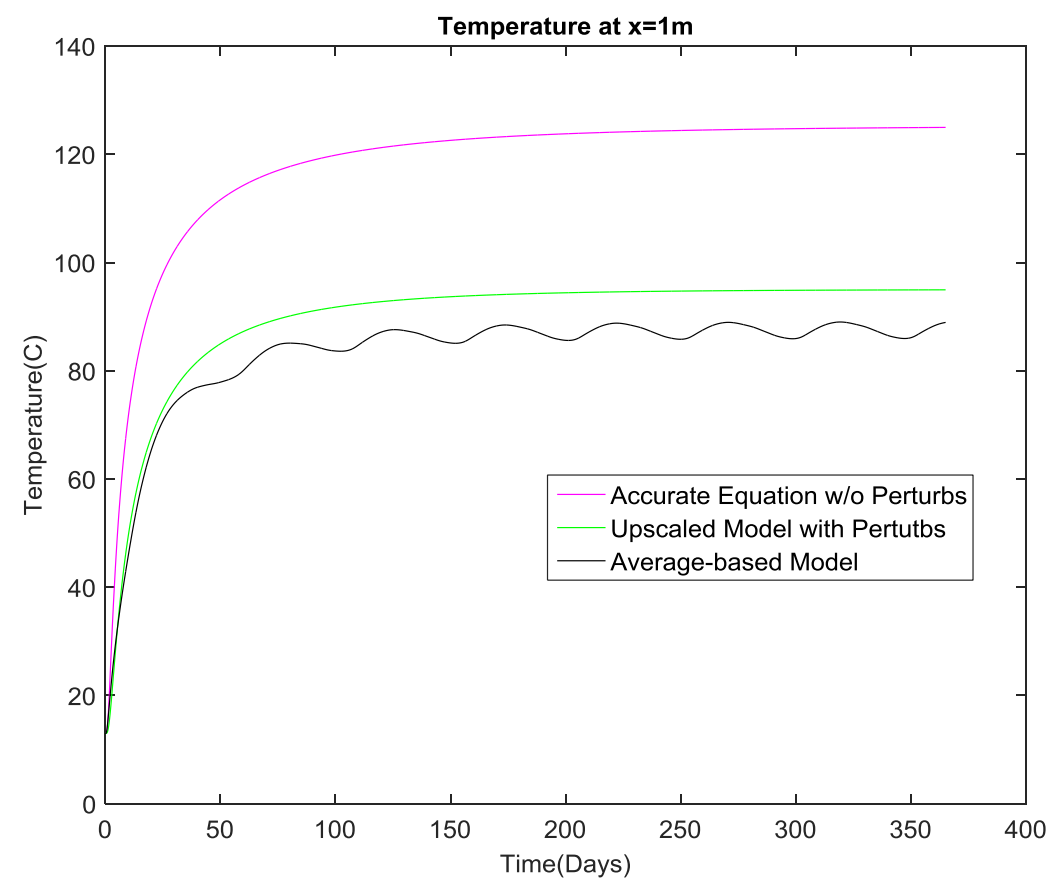

Figure 4-11: Comparison of developed upscaled model with Butler's model and average-based model of 100 realizations for $a_{\alpha}=0.9$ and $\omega=\pi$

Finally, in the last run, the effect of velocity of interface is examined. In Figures......, $U$ is changed from $0.8 \times 4.76 \times 10^{-7}$ to $1.4 \times 4.76 \times 10^{-7} \frac{\mathrm{m}}{\mathrm{s}}$. The results show that as this parameter increases, the temperature curve oscillate more rapidly. These oscillations are caused by the harmonic nature of distributions assigned to the diffusivities. This property at a specific point at time $\mathrm{t}$ can be written as:

$$
\alpha(x)=\bar{\alpha}+a \cos (\omega \times(U . t+\eta)+\phi)
$$

As seen, velocity of boundary has a positive correlation with the frequency of this property. This observation may be better described if compared with a driving example. Imagine a driver runs the car on a road that is full of bumps. As he/she speeds up he/she will pass the bumps more frequently. The spatial location of bumps are fixed and do not change but the drivers with different speeds pass them with different frequencies. 


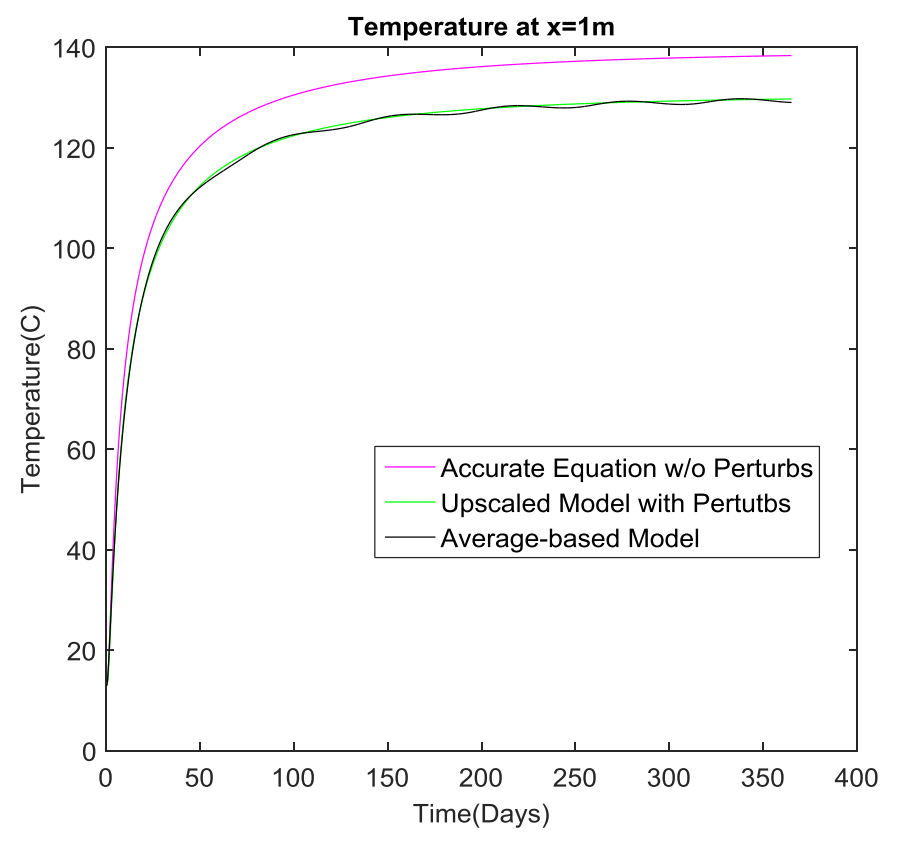

Figure 4-12: Comparison of developed upscaled model with Butler's model and average-based model of 100 realizations for $a_{\alpha}=0.6, \omega=\pi$ and $U=0.8 \times 4.76 \times 10^{-7} \frac{\mathrm{m}}{\mathrm{s}}$

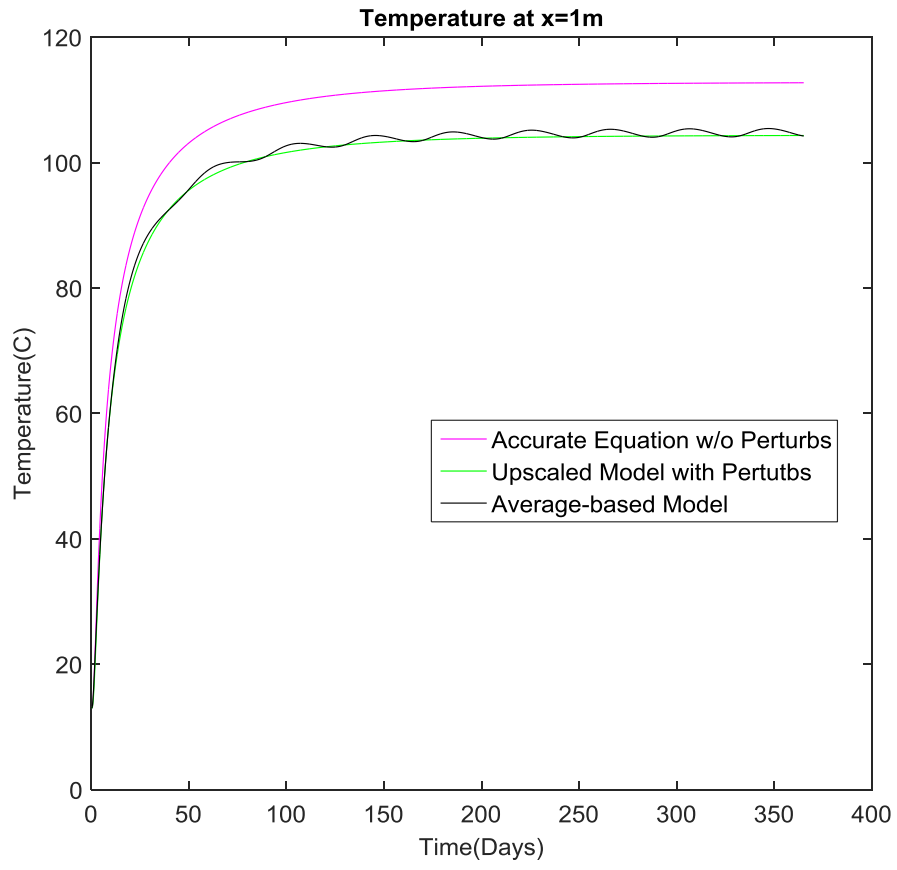

Figure 4-13: Comparison of developed upscaled model with Butler's model and average-based model of 100 realizations for $a_{\alpha}=0.6, \omega=\pi$ and $U=1.2 \times 4.76 \times 10^{-7} \frac{\mathrm{m}}{\mathrm{s}}$ 


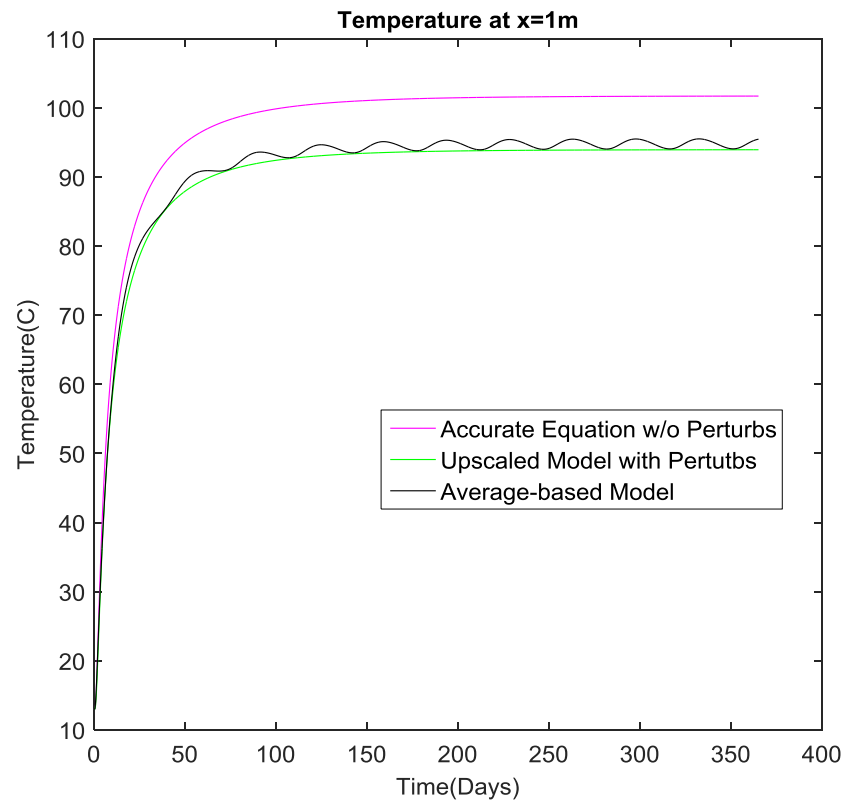

Figure 4-14: Comparison of developed upscaled model with Butler's model and average-based model of 100 realizations for $a_{\alpha}=0.6, \omega=\pi$ and $U=1.4 \times 4.76 \times 10^{-7} \frac{\mathrm{m}}{\mathrm{s}}$

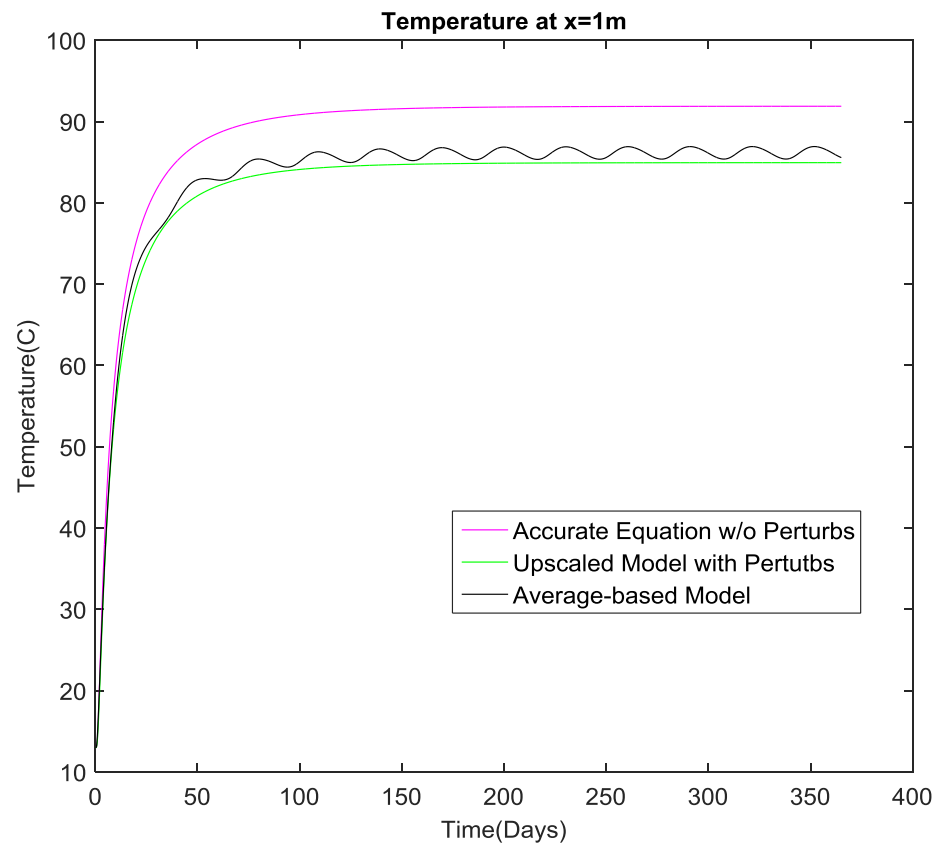

Figure 4-15: Comparison of developed upscaled model with Butler's model and average-based model of 100 realizations for $a_{\alpha}=0.6, \omega=\pi$ and $U=16 \times 4.76 \times 10^{-7} \frac{\mathrm{m}}{\mathrm{s}}$ 


\section{Chapter 5. Results}

\subsection{Comparison with Butler's method}

To develop the equations obtained in previous section, we made a couple of assumptions and simplifications that are different from the ones made by Butler. One of these differences is related to parameter $\mathrm{m}$. Butler defined $\mathrm{m}$ as a parameter that depends on the fluid properties and reservoir and steam temperatures. But in our developed equation, viscosity and temperature are correlated based on Eq. 3-7. There are 6 parameters appear in the final equation of drainage rate, VV, MM, NN, LL, TT, and BB. To compare our results with Butler's, parameter m should be calculated. Based on the value of $\mathrm{m}, \epsilon_{e q q}$ should be obtained.

\subsubsection{Parameter $\boldsymbol{m}$ calculation}

Parameter $m$ indicates the relationship between viscosity and temperature for the fluid and appears in Butler's equation. For three types of crudes that are distinguished by viscosity in $100{ }^{\circ} \mathrm{C}$ and $200{ }^{\circ} \mathrm{C}$, Figure 5-1 shows the parameter of $\mathrm{m}$ in different steam temperatures and fixed reservoir temperature $\operatorname{Tr}=13{ }^{\circ} \mathrm{C} .{ }^{\circ} \mathrm{C}$

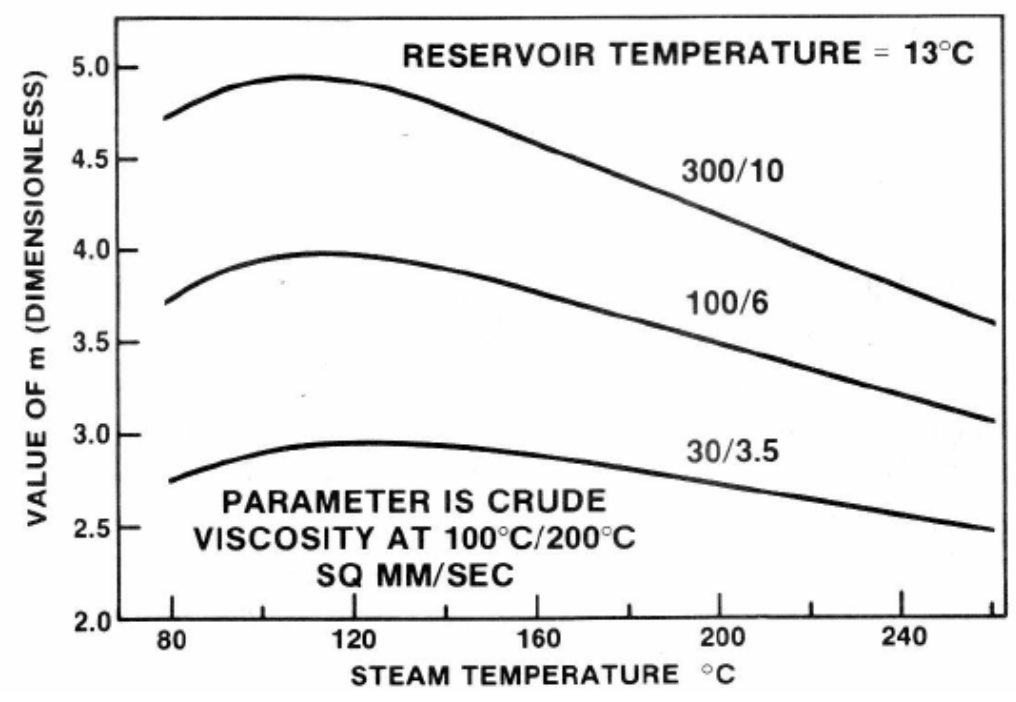

Figure 5-1: Boundary value of $m$ for different fluid oil [2] 
In our case study, the viscosity at 100 and $200{ }^{\circ} \mathrm{C}$ is $129 / 6.8$, and steam temperature is $215{ }^{\circ} \mathrm{C}$. Based on this information, according to the chart in Figure 5-1, m will be equal to 3.7 .

\subsubsection{Consistency with Butler's Model}

As previously mentioned, the flow rate is produced along the reservoir ahead of steam interface and if there is no limit in integrals used to develop our equations, flowrate will be converge to infinity. To avoid this problem, the interval of integral where the fluid flow is produced will be limited. This can be done by introducing a small number to reservoir temperature named as $\epsilon_{e q q}$. It indicates that the integral considers only a limited region.

Butler used another method to avoid this problem in his method [2]. As in this section we are looking for a comparison between the methods, we need to find the proper value of $\epsilon_{e q q}$ that gives the same results. Butler's equation for the flow rate in SAGD is:

$$
\bar{q}=\sqrt{2(\alpha) \frac{K_{L} g}{m v_{s}} * \bar{\phi} \Delta S_{o} * h}
$$

According to Butler [2Where $\mathrm{m}$ is defined as:

$$
m=\left[v_{S} \int_{T_{R}}^{T_{S}}\left(\frac{1}{v}-\frac{1}{v_{R}}\right) \frac{d T}{T-T_{R}}\right]^{-1}
$$

Our general developed equation for flow rate is:

$$
\bar{q}=\sqrt{2(\bar{\alpha}) \frac{K_{L} g}{v_{R}} * \bar{\phi} \Delta S_{o} * h * A A}
$$

If case study is deterministic, Eq. 5-3 becomes:

$$
\bar{q}=\sqrt{2(\bar{\alpha}) \frac{K_{L} g}{v_{R}} * \bar{\phi} \Delta S_{o} * h * \int_{T_{S}}^{T_{R}+\epsilon} \frac{e^{\beta\left(\bar{T}-T_{R}\right)} d \bar{T}}{\left(\bar{T}-T_{R}\right)}}
$$


If the flow rate achieved by two different methods are set to be equal:

$$
\int_{T_{S}}^{T_{R}+\epsilon} \frac{e^{\beta\left(\bar{T}-T_{R}\right)} d \bar{T}}{\left(\bar{T}-T_{R}\right)}=\frac{1}{m v_{S}}
$$

Substituting the value of $\mathrm{m}$ (Eq. 5-2) in this equation, yields:

$$
\int_{T_{S}}^{T_{R}+\epsilon} \frac{e^{\beta\left(\bar{T}-T_{R}\right)} d \bar{T}}{\left(\bar{T}-T_{R}\right)}=\int_{T_{R}}^{T_{S}}\left(\frac{1}{v}-\frac{1}{v_{R}}\right) \frac{d T}{T-T_{R}}
$$

The solution of this integral at the left side is:

$$
\left[\operatorname{Ei}(\beta \epsilon)-\operatorname{Ei}\left(\beta *\left(T_{S}-T_{R}\right)\right)\right]
$$

Right side can be written as:

$$
\int_{T_{R}}^{T_{S}}\left(\frac{1}{v}-\frac{1}{v_{R}}\right) \frac{d T}{T-T_{R}}=\int_{T_{S}}^{T_{R}}\left(\frac{1}{v_{R}}-\frac{1}{v}\right) \frac{d T}{T-T_{R}}=\int_{T_{S}}^{T_{R}}\left(\frac{1}{v_{R}}-\frac{\left(e^{\beta\left(T_{R}-T\right)}\right.}{v_{R}}\right) \frac{d T}{T-T_{R}}
$$

With equating these two equations, an equation is developed for $\epsilon$ :

$$
E i(\beta \epsilon)=E i\left(\beta *\left(T_{S}-T_{R}\right)\right)+v_{R} \int_{T_{S}}^{T_{R}}\left(\frac{1}{v_{R}}-\frac{\left(e^{\beta\left(T_{R}-T\right)}\right.}{v_{R}}\right) \frac{d T}{T-T_{R}}
$$

By solving this equation, the equivalent value of $\epsilon$ is achieved as

$$
\epsilon_{e q}=1.63 * 10^{-8}{ }^{\circ} \mathrm{C}
$$

This values of $\epsilon$ corresponds to a distance to:

$$
\xi_{\text {eq }} \cong 40 \mathrm{~m}
$$

The flow rates in SAGD process vary from 0.05 to $0.8 \frac{\mathrm{m}^{3}}{\mathrm{~m} . \mathrm{day}}$ per meter for horizontal well (Butler [2]). 


\subsection{Evaluating the developed flow equation for typical values}

In this section, the typical values of variables of flow rate equation will be used to evaluate the effect of heterogeneity on flow rate. In this evaluation, the thermal diffusivity is set to have $50 \%$ perturbation. The similar steps should be done for other values of perturbation.

\subsubsection{Viscosity}

A typical crude oil has a viciously of $129.1 \frac{\mathrm{mm}^{2}}{\mathrm{~s}}$ (or cs) at $100^{0} \mathrm{C}$ and $6.8 \frac{\mathrm{mm}^{2}}{\mathrm{~s}}$ at $200^{0} \mathrm{C}$ (Butler [2].

$$
1 \text { centisrokes }=1 \mathrm{~mm}^{2} \mathrm{~s}^{-1}=10^{-6} \mathrm{~m}^{2} \mathrm{~s}^{-1}
$$

Therefore, typical values in SI unit, for these values will be $129.1 * 10^{-6} \frac{\mathrm{m}^{2}}{\mathrm{~s}}$ and $6.8 * 10^{-6} \frac{\mathrm{m}^{2}}{\mathrm{~s}}$ at $100^{\circ} \mathrm{C}$ and $200^{\circ} \mathrm{C}$.

\subsubsection{Permeability}

A typical reservoir of heavy oil can have a $0.4 d$ effective permeability for oil flow (Butler [2])

1 Darcy unit in SI unit is equal to $=9.869233 \times 10^{-13} \mathrm{~m}^{2} \approx 10^{-12} \mathrm{~m}^{2}$

$$
\begin{gathered}
\Rightarrow 0.4 d=0.4 * 10^{-12} \mathrm{~m}^{2} \\
K_{L}=0.4 * 10^{-12} \mathrm{~m}^{2}
\end{gathered}
$$

\subsubsection{Reservoir and Steam Temperature}

$T_{s}=215^{\circ} \mathrm{C}$ and $T_{r}=13^{\circ} \mathrm{C}$.

\subsubsection{Saturation}

A typical residual and oil saturation can be as:

$$
S_{r}=0.13 \text { and } S_{o}=0.75
$$

\subsubsection{Porosity}

The typical value of this property can be as: 


$$
\phi=0.33
$$

\subsubsection{Thermal Diffusivity}

$\alpha=8.10 * 10^{-7} \frac{\mathrm{m}^{2}}{\mathrm{~s}}$ and we take for this specific example $a=0.5 * 8.10 * 10^{-7} \frac{\mathrm{m}^{2}}{\mathrm{~s}}$.

Where $a$ is indicates the range of heterogeneity. Thermal diffusivity varies within [-a,a].

\subsubsection{Temperature and Viscosity Correlation}

$$
\begin{gathered}
\operatorname{Ln} \frac{1}{v}=\overline{m(x)}+m^{\prime}(x)=\operatorname{Ln}\left(\frac{1}{v_{R}}\right)+\beta\left(\bar{T}-T_{R}\right)+\beta T^{\prime}: \quad \beta>0 \\
\frac{1}{v_{L}}=e^{\overline{m(x)}}=\frac{1}{v_{R}} \times e^{\beta\left(\bar{T}-T_{R}\right)} \\
\Rightarrow \beta=\frac{1}{\left(T_{2}-T_{1}\right)} \ln \left(\frac{v_{1}}{v_{2}}\right)=\frac{1}{(200-100)} \ln \left(\frac{129.1}{6.8}\right)=0.0294
\end{gathered}
$$

\subsubsection{Viscosity of Fluid at Reservoir Temperature}

Viscosity of fluid at reservoir temperature can be calculated based on the correlation introduced by Eq. 5-7.

$$
\begin{gathered}
\frac{1}{v_{R}}=\frac{1}{v_{1}} \times e^{\beta\left(T_{R}-T_{1}\right)}=\frac{1}{129.1 * 10^{-6}} * e^{0.0294 *(13-200)}=598 \\
\Rightarrow v_{R}=\frac{1}{4.7894 * 10^{14}}=0.0017 \frac{\mathrm{m}^{2}}{\mathrm{~s}}
\end{gathered}
$$

\subsubsection{Velocity of Boundary}

In this case study, the velocity of boundary is supposed to be

$$
U=4.76 * 10^{-7} \mathrm{~m} / \mathrm{s}
$$

Now that all the variables are known, parameter AA can be calculated.

$$
A A=[V V \times L L+M M \times T T+N N \times B B]
$$

Where: 


$$
\begin{gathered}
V V=\left(1+\frac{1}{2} \int_{-\infty}^{+\infty} c^{2} S_{\alpha \alpha} d k\right) \\
M M=\left(\frac{1}{2} \int_{-\infty}^{+\infty} \frac{\beta^{2} B^{4}}{\bar{\alpha}^{2} k^{4}} S_{\alpha \alpha} d k\right) \\
N N=\left(\int_{-\infty}^{+\infty} \frac{\beta c B^{2}}{\bar{\alpha} k^{2}} S_{\alpha \alpha} d k\right) \\
L L=\int_{T_{S}}^{T_{R}+\epsilon} \frac{e^{\beta\left(\bar{T}-T_{R}\right)} d \bar{T}}{\left(\bar{T}-T_{R}\right)} \\
T T=\int_{T_{S}}^{T_{R}+\epsilon} e^{\beta\left(\bar{T}-T_{R}\right)}\left(\bar{T}-T_{R}\right) d \bar{T} \\
B B=\int_{T_{S}}^{T_{R}+\epsilon} e^{\beta\left(\bar{T}-T_{R}\right)} d \bar{T}
\end{gathered}
$$

C, B and $S_{\alpha \alpha}$ should be determined.

5.2.10. Parameter $c$ Calculation

$$
\begin{aligned}
\operatorname{Ln} k(x) & =f(x)=\bar{f}+f^{\prime}(x)=\bar{f}+\bar{f} a_{f} \cos \left(\omega_{k} x+\theta\right) \\
\alpha & =\bar{\alpha}+\alpha^{\prime}(x)=\bar{\alpha}+\bar{\alpha} a_{\alpha} \cos \left(\omega_{k} x+\theta\right)
\end{aligned}
$$

For the permeability:

$$
\begin{gathered}
\bar{f}=\operatorname{Ln} K_{L} \\
f^{\prime}=c * \alpha^{\prime}(x) \Rightarrow \bar{f} a_{f} \cos \left(\omega_{k} x+\theta\right)=c \bar{\alpha} a_{\alpha} \cos \left(\omega_{k} x+\theta\right) \\
\Rightarrow a_{f}=c \frac{\bar{\alpha} a_{\alpha}}{\bar{f}}
\end{gathered}
$$

Eq. 5-13 can be written as:

$$
\operatorname{Ln} k(x)=\operatorname{Ln} K_{L}+\bar{f} a_{f} \cos \left(\omega_{k} x+\theta\right)
$$

If the critical points of $\mathrm{k}$ is written as:

$$
\ln \left(K_{L}+K_{L} a_{k}\right)=\operatorname{Ln} K_{L}+\bar{f} a_{f}=\operatorname{Ln} K_{L}+c \bar{\alpha}
$$




$$
\Rightarrow \operatorname{Ln}\left(1+a_{k}\right)=c \bar{\alpha}
$$

For positive correlations, $\mathrm{c}$ is positive and for negative correlations, $\mathrm{c}$ will be negative.

\subsubsection{Parameter $B$ Calculation}

Value of B is dependent on the spectrum of thermal diffusivity. The general equation for the B is:

$$
\begin{gathered}
B=\frac{U}{\alpha_{e q}} \\
\text { where } \alpha_{e q}=\bar{\alpha}-\int_{-\infty}^{\infty} \frac{1}{\bar{\alpha}} S_{\alpha \alpha} d k
\end{gathered}
$$

For our specific case that harmonic distribution is used for $\alpha$, parameter $\mathrm{B}$ will be:

$$
B_{\text {harmonic }}=\frac{U}{\bar{\alpha}-\frac{a^{2}}{\bar{\alpha}}}=\frac{4.76 * 10^{-7} \mathrm{~m} / \mathrm{s}}{8.10 * 10^{-7} \frac{\mathrm{m}^{2}}{\mathrm{~s}}-\frac{\left(\frac{1}{2} * 8.10 * 10^{-7} \frac{\mathrm{m}^{2}}{\mathrm{~s}}\right)^{2}}{8.10 * 10^{-7} \frac{\mathrm{m}^{2}}{\mathrm{~s}}}}=0.7835 \mathrm{~m}^{-1}
$$

\subsubsection{Spectrum of thermal diffusivity}

$$
\begin{aligned}
S_{a a}=\frac{1}{4} a^{2}[\delta(k & \left.\left.+k_{0}\right)+\delta\left(k-k_{0}\right)\right]=S_{a a} \\
& =\frac{1}{4}\left(\frac{1}{2} * 8.10 * 10^{-7} \frac{m^{2}}{s}\right)^{2}\left[\delta\left(k+\frac{\pi}{2}\right)+\delta\left(k-\frac{\pi}{2}\right)\right] \\
& =4.1 * 10^{-14}\left[\delta\left(k+\frac{\pi}{2}\right)+\delta\left(k-\frac{\pi}{2}\right)\right]
\end{aligned}
$$

Now, VV, MM and NN can be calculated:

$$
\begin{gathered}
V V=\left(1+\frac{1}{2} \int_{-\infty}^{+\infty} c^{2} S_{\alpha \alpha} d k\right)=1 . .0358 \\
M M=\left(\frac{1}{2} \int_{-\infty}^{+\infty} \frac{\beta^{2} B^{4}}{\bar{\alpha}^{2} k^{4}} S_{\alpha \alpha} d k\right)=3.352 * 10^{-6} \\
N N=\left(\int_{-\infty}^{+\infty} \frac{\beta c B^{2}}{\bar{\alpha} k^{2}} S_{\alpha \alpha} d k\right)=6.9295 * 10^{-4}
\end{gathered}
$$




$$
\begin{gathered}
L L=\int_{T_{S}}^{T_{R}+\epsilon} \frac{e^{\beta\left(\bar{T}-T_{R}\right)} d \bar{T}}{\left(\bar{T}-T_{R}\right)}=\left[E i(\beta \epsilon)-E i\left(T_{S}-T_{R}\right)\right]=-103.3328 \\
T T=\int_{T_{S}}^{T_{R}+\epsilon} e^{\beta\left(\bar{T}-T_{R}\right)}\left(\bar{T}-T_{R}\right) d \bar{T}=\left[\frac{e^{\beta \epsilon}(\beta \epsilon-1)}{\beta^{2}}-\frac{\left(e^{\beta\left(T_{S}-T_{R}\right)}\left(\beta\left(T_{S}-T_{R}\right)-1\right)\right.}{\beta^{2}}\right] \\
=-2.18 * 10^{6} \\
B B=\int_{T_{S}}^{T_{R}+\epsilon} e^{\beta\left(\bar{T}-T_{R}\right)} d \bar{T}=\frac{1}{\beta}\left(e^{\beta \epsilon}-e^{\beta\left(T_{S}-T_{R}\right)}\right)=-4.1488 * 10^{43} \\
\Rightarrow A A=-1.29 * 10^{4}
\end{gathered}
$$

Now the integrals should be calculated as:

$$
\overline{q_{\text {harmonic }}}=\sqrt{2\left(\bar{\alpha}-\frac{a^{2}}{\bar{\alpha}}\right) \frac{K_{L} g}{v_{R}} * \bar{\phi} \Delta S_{o} * h * A A}=0.1095 \frac{m^{2}}{s}
$$

Flow rates from about 0.025 to $0.4 \frac{m^{3}}{m . d a y}$ per meter for horizontal well is predicted from one side.

\subsection{Correlated Flow Rate Curves}

In this section, all the information previously discussed in Chapter 3 is fed into the developed SAGD model. The results show that heterogeneity of thermal diffusivity has a very large effect on the produced flow rate. Variations of this property, has a remarkable negative effect on the produced flow rate in the process. This is probably due to its negative impact on the conduction of heat in the reservoir that is the underlying basic in In Situ recovery methods.

As already mentioned, to simulate the heterogeneities of reservoir, a harmonic random field has been assigned to permeability and thermal diffusivity perturbations. The total values of these properties can be written as:

$$
\begin{gathered}
\operatorname{Ln} k(x)=f(x)=\bar{f}+f^{\prime}(x)=\bar{f}+\bar{f} a_{f} \cos \left(\omega_{k} x+\theta\right) \\
\alpha=\bar{\alpha}+\alpha^{\prime}(x)=\bar{\alpha}+\bar{\alpha} a_{\alpha} \cos \left(\omega_{k} x+\theta\right)
\end{gathered}
$$


According to Eq. 5-13, $\mathrm{k}(\mathrm{x})$ will be a periodic function. $a_{k}$ is defined such that permeability maximum values are at these points:

$$
K_{L}+K_{L} a_{k}
$$

Parameter c as already calculated in Eq. 5-12 is equal to:

$$
c= \pm \frac{\operatorname{Ln}\left(1+a_{k}\right)}{\bar{\alpha}}
$$

Positive and negative signs indicate positive and negative correlations respectively. $a_{k}$ in this equation is always positive. In Figure 5-2, the discussed properties including the mean and their harmonic variables are shown.

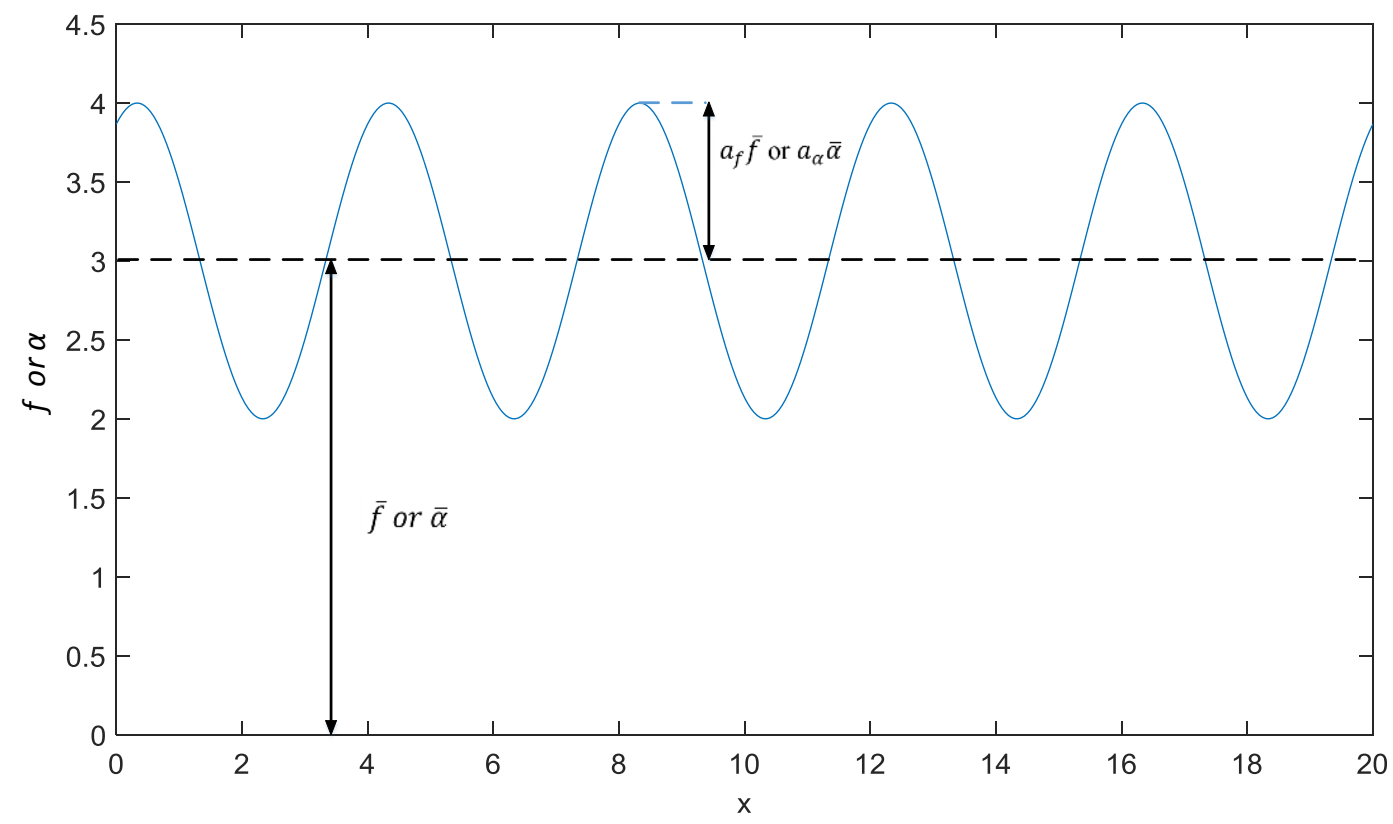

Figure 5-2: Heterogeneous properties are assumed to be the sum of their mean and harmonic perturbation.

The frequency and the phase for both of properties are assumed identical. But the sign of the amplitude ratios $\left(a_{f}\right.$ and $a_{\alpha}$ ), depending on whether the correlation is positive or negative may be identical or opposite. 
Figure 5-3 shows how the flow is affected with heterogeneity in permeability and thermal diffusivity when there is a positive correlation between these properties. The results suggest that, the thermal diffusivity has a major negative impact on the efficiency of the SAGD whereas permeability diffusivity shows a subtle effect that is initially negative but surprisingly from a specific point later, its effect becomes positive. For instance, the positive effect of permeability perturbation is clearly observed in the case that diffusivity has a $10 \%$ perturbation and permeability has a positive effect on flow rate from $13 \%$ up to the point that in $32 \%$, it overpasses homogeneous solution. This observation shows that perturbation is not always unfavorable. In some cases, depending on the governing equations, and how the inputs and outputs of the process or reservoir are correlated, the perturbation can yield better results. In SAGD, variation in thermal diffusivity decreases the temperature along the reservoir compared to homogeneous SAGD. This reduces the produced flow rate. On the other hand, perturbation of permeability can either increase or decrease the flowrate depending on size of permeability heterogeneities and thermal diffusivity.

In Figure 5-3, the correlation of properties is positive whereas Figure 5-4 shows this effect for the negative correlation. As seen, in Figure 5-3, permeability perturbations increases the flow rate for all the cases and even under some specific conditions it overpasses the unperturbed solution. This diagram can be created based on the information available for any reservoir and $b$ used as a guideline to predict the flow rate of SAGD.

AA is one of the important parameters in developed equation for flow rate (Eq. 3-39) that include the heterogeneities values. Figure 5-5 shows the value of this parameter against different values of heterogeneities. This type of diagram can be made for any reservoir and be used as a reference to predict the produced drainage rate. Figure 5-6 depicts the similar curves for the negative correlation.

Figure 5-7 and Figure 5-8 show the produced flow rate against the both heterogeneities for positive and negative correlation. In Figure 5-9 and Figure 5-10, it is shown that how the flow rate in SAGD is influenced by the heterogeneities in the reservoir against different values of parameter c. In Figure 5-9, where positive correlation is assumed, increasing correlation constant $\mathrm{c}$ and subsequently increasing of permeability perturbation, increases the flow rate and neutralizes the effect of perturbation in thermal diffusivity. Figure 5-8 includes similar flow rate curve but with a negative correlation between heterogeneities. Assigning negative values to parameter $\mathrm{c}$, makes 
permeability and diffusivity correlation to be negative. The results shown in this figure, suggest that increasing the permeability, reduces the produced flow rate if the correlation between heterogeneities is negative while in previous case with positive correlation, increasing the permeability improves the efficiency of SAGD and increases the flow rate. This observation is consistent with previous curves shown by Figure 5-3, Figure 5-4, Figure 5-5, Figure 5-6, Figure 5-7 and Figure 5-8. 


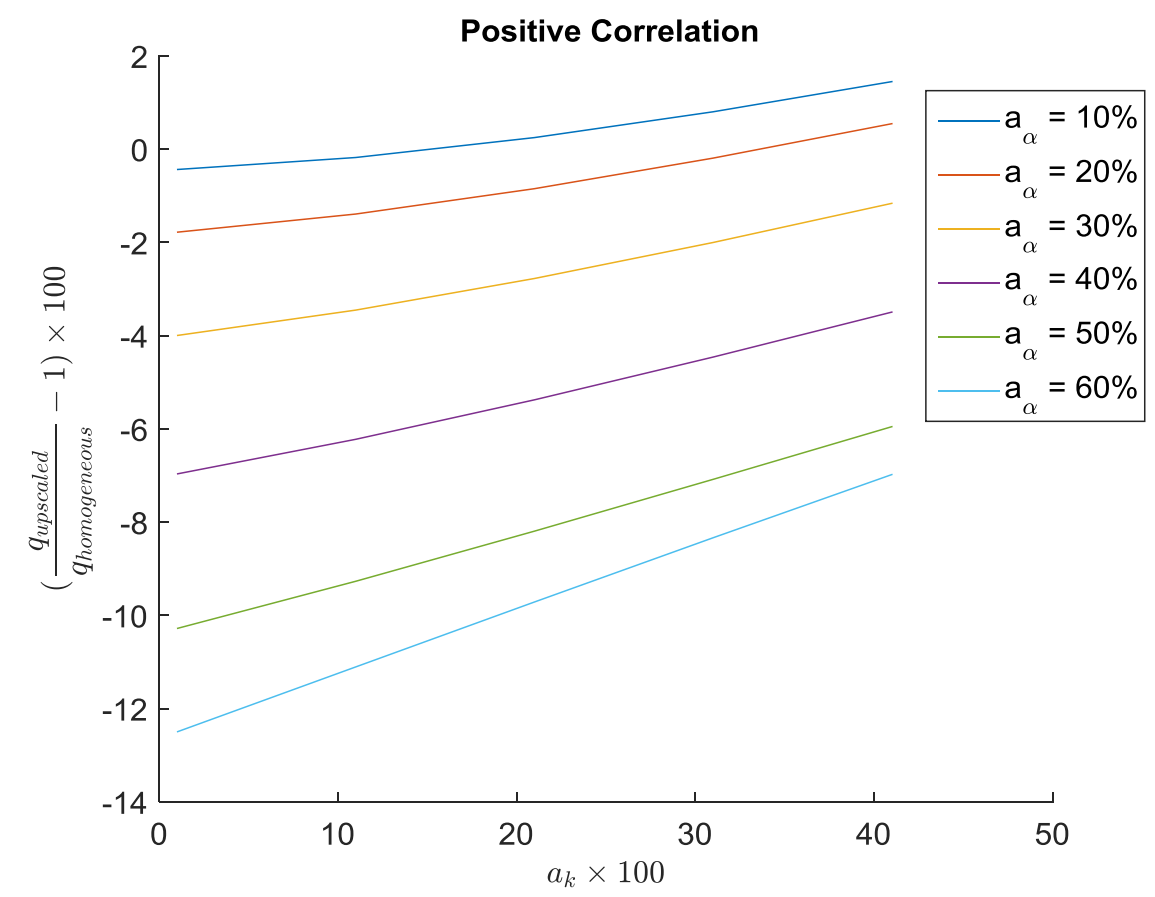

Figure 5-3: Effect of heterogeneities on the flow rate for positive correlation between $\mathrm{k}$ and $\alpha$

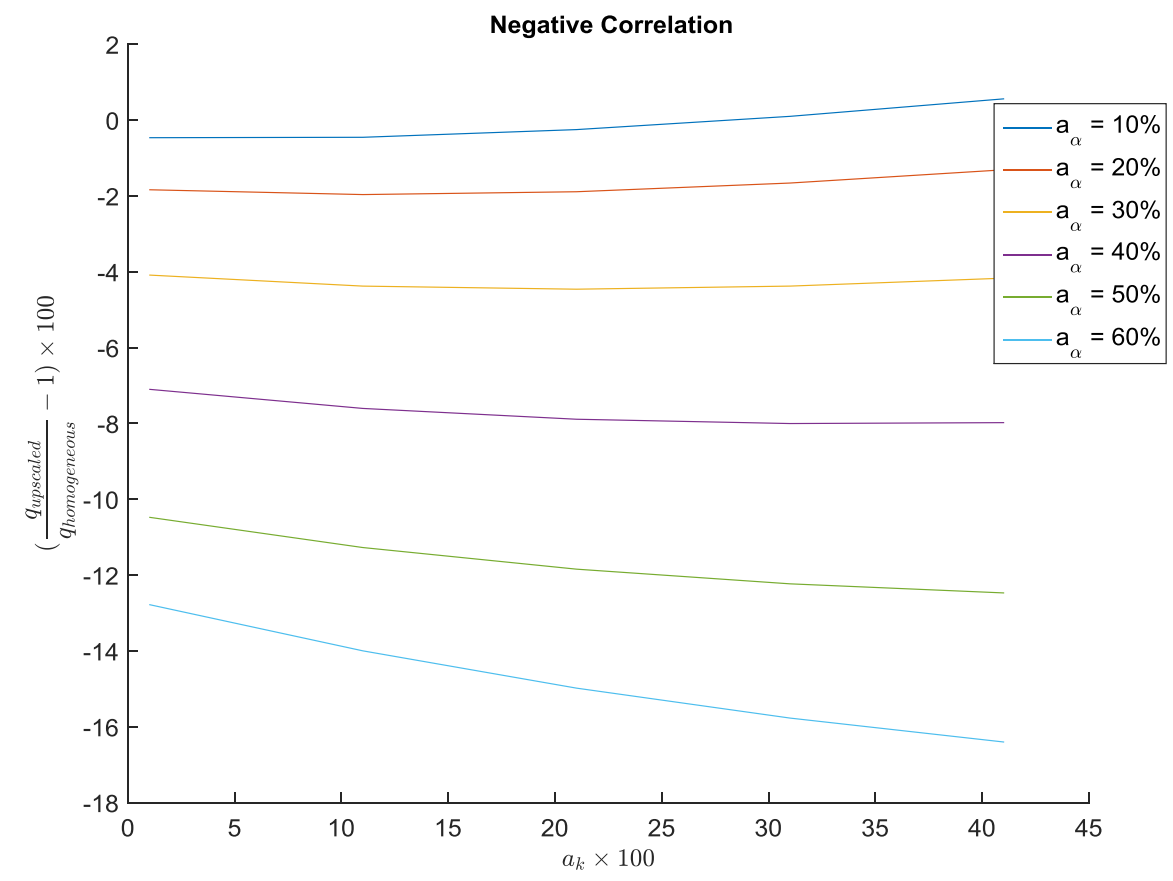

Figure 5-4: Effect of heterogeneities on the flow rate for negative correlation between $\mathrm{k}$ and $\alpha$ 


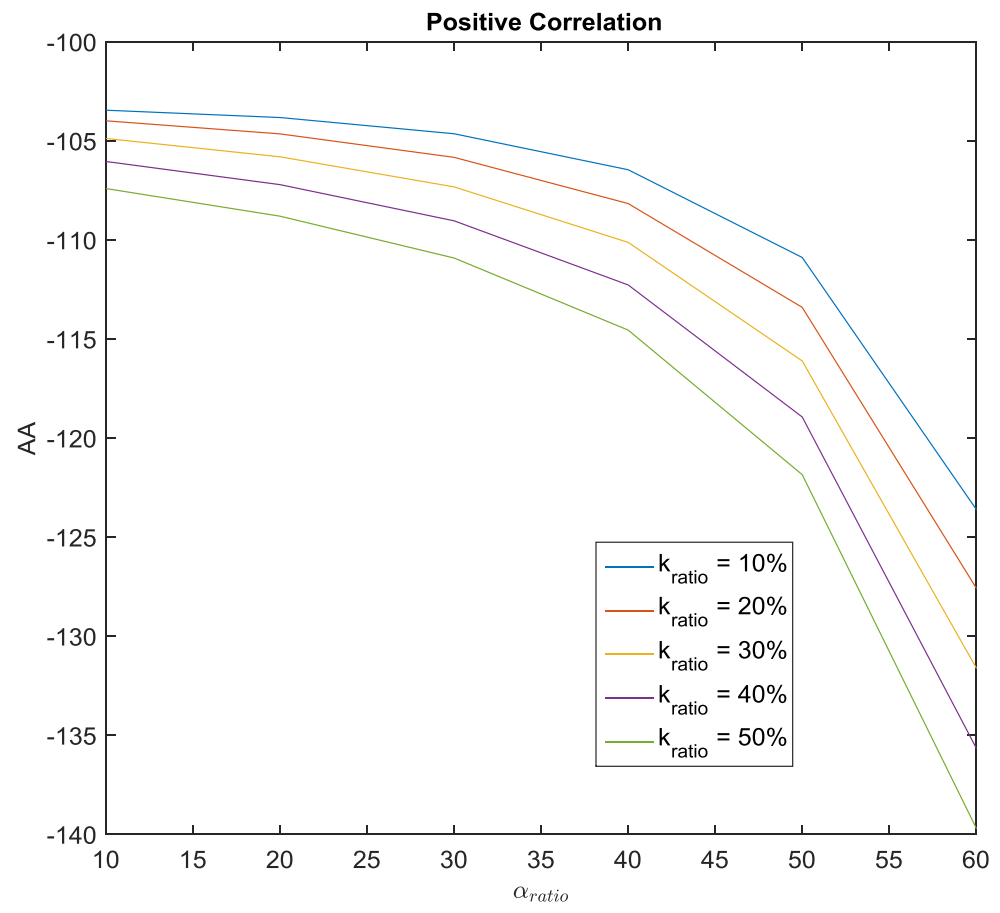

Figure 5-5: Values of AA against reservoir heterogeneities for positive correlation

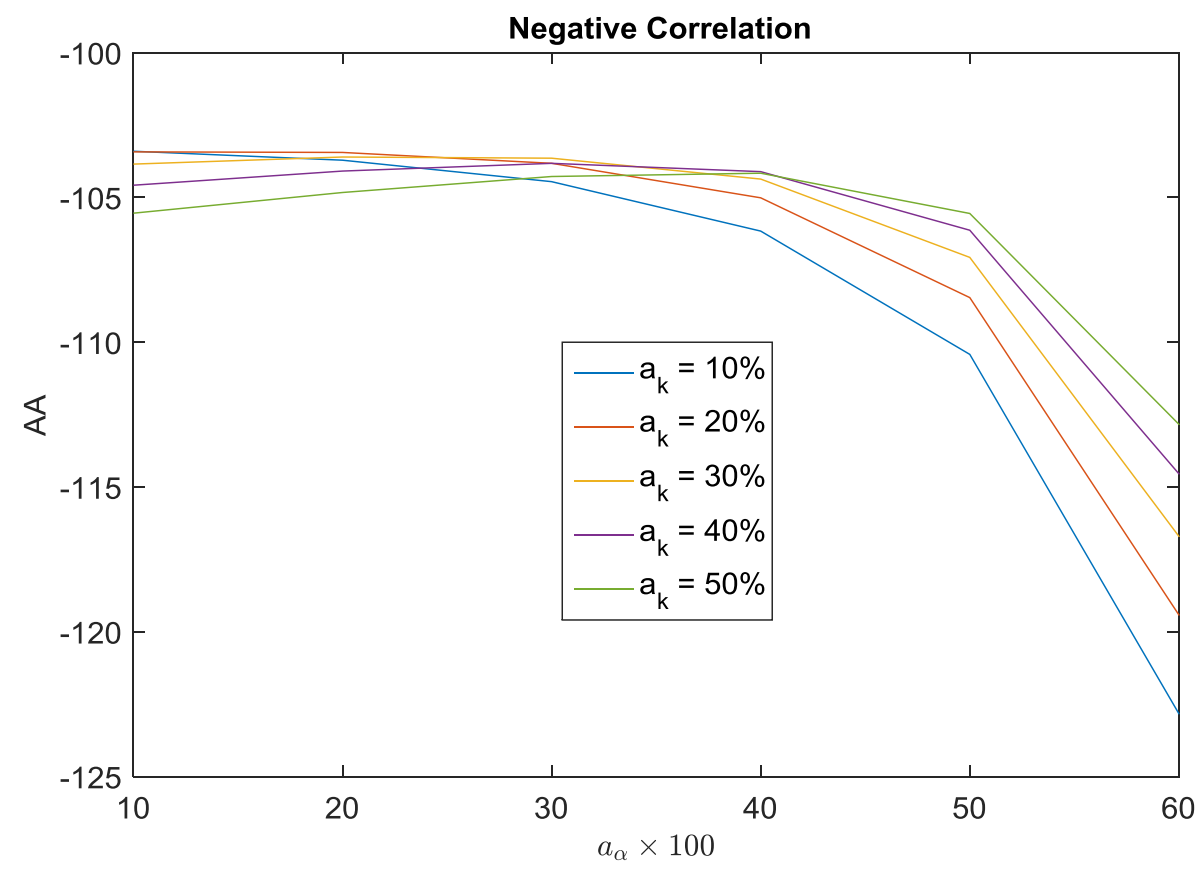

Figure 5-6: Values of AA against reservoir heterogeneities for negative correlation 


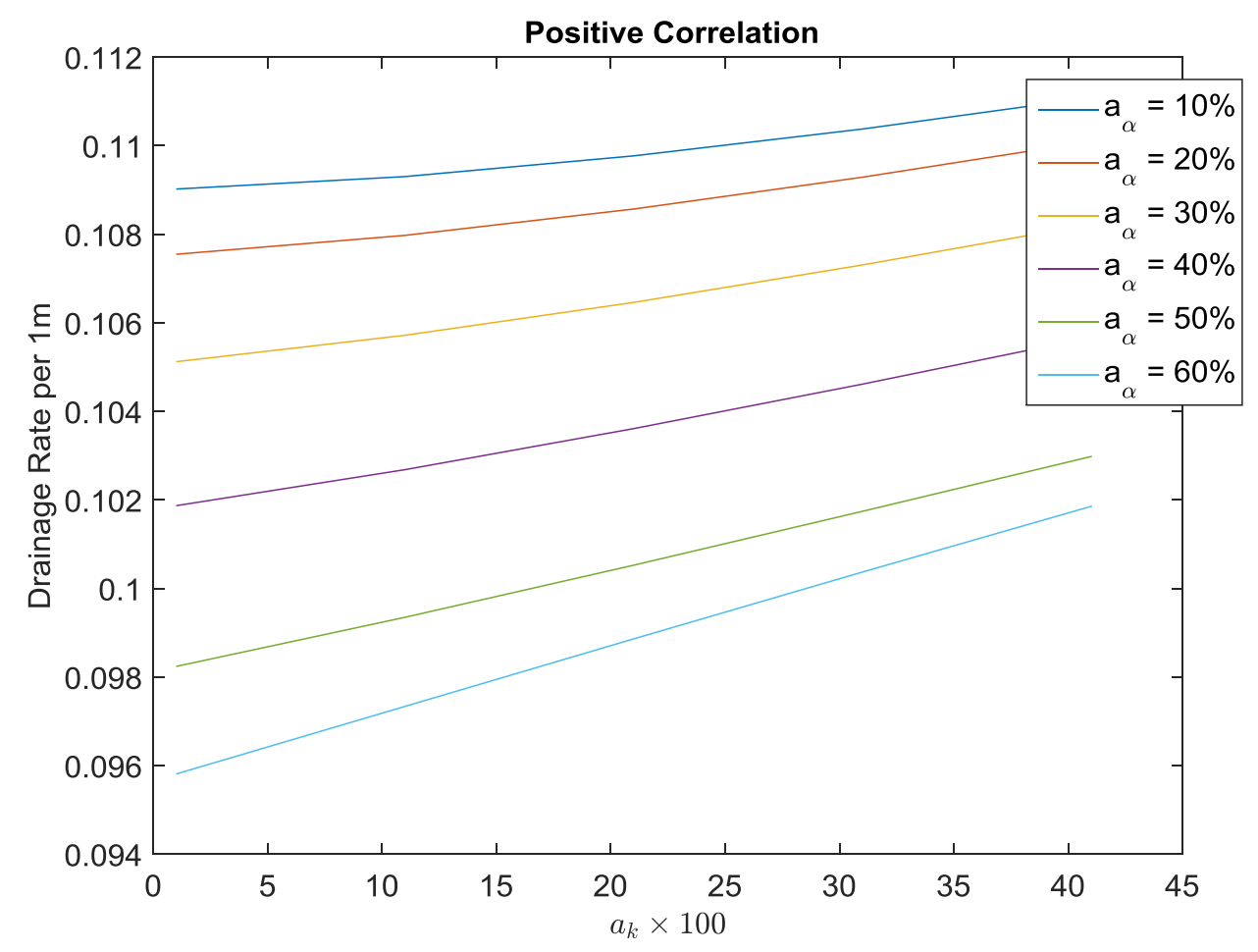

Figure 5-7: Flow rate in SAGD for positive correlation

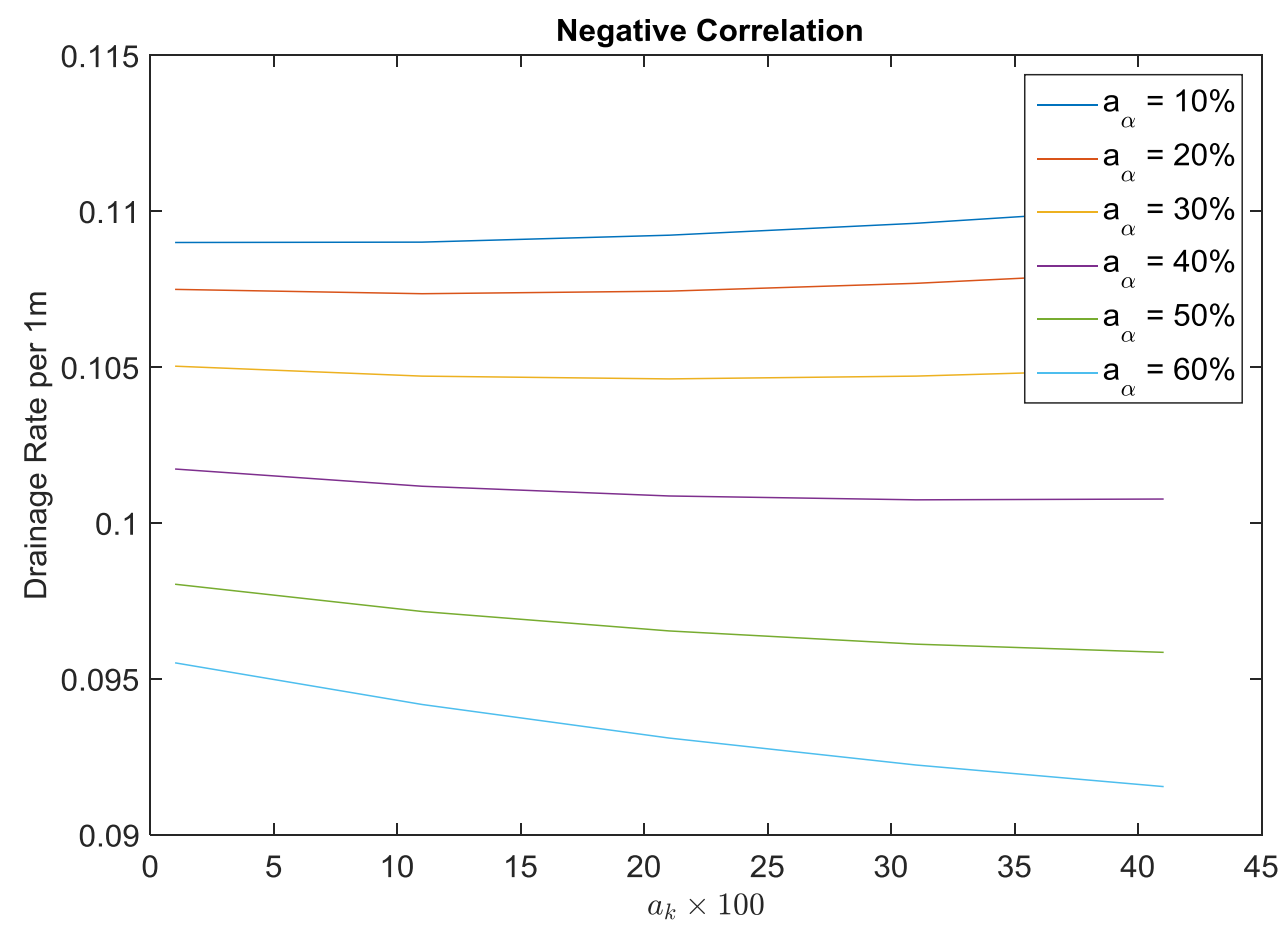

Figure 5-8: Produced flow rate in SAGD for negative correlation 


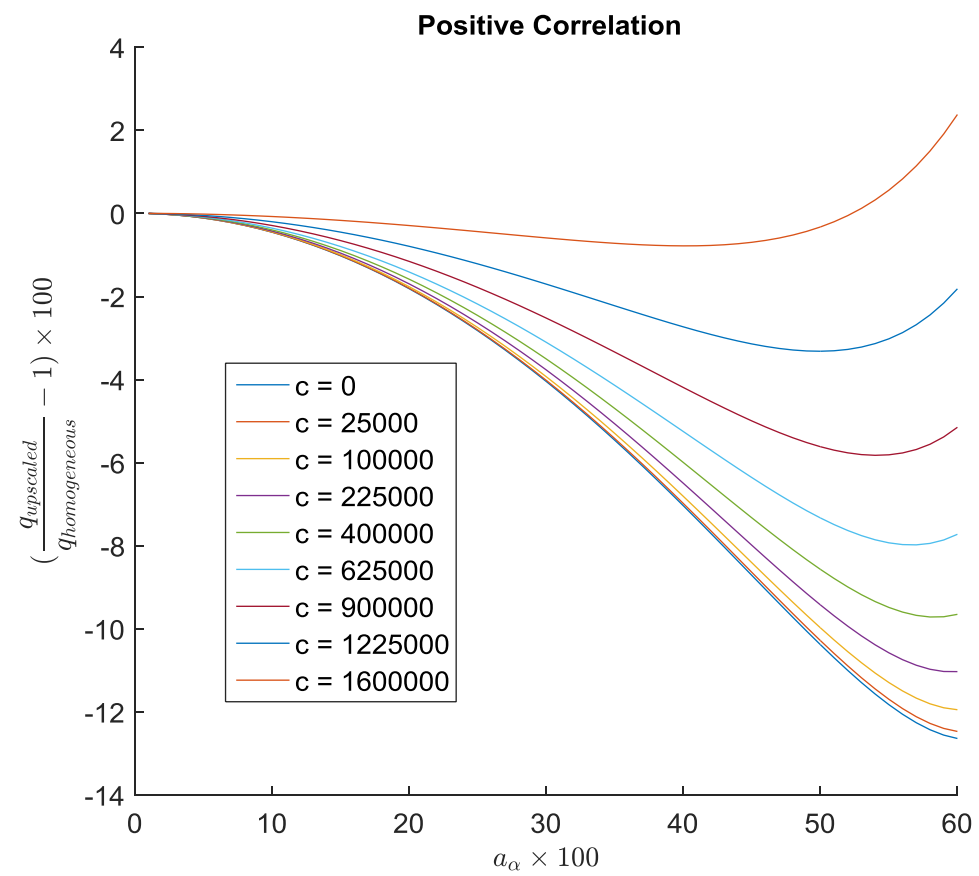

Figure 5-9: Effect of heterogeneities on the flow rate against constant values of $\mathrm{c}$ for positive correlation

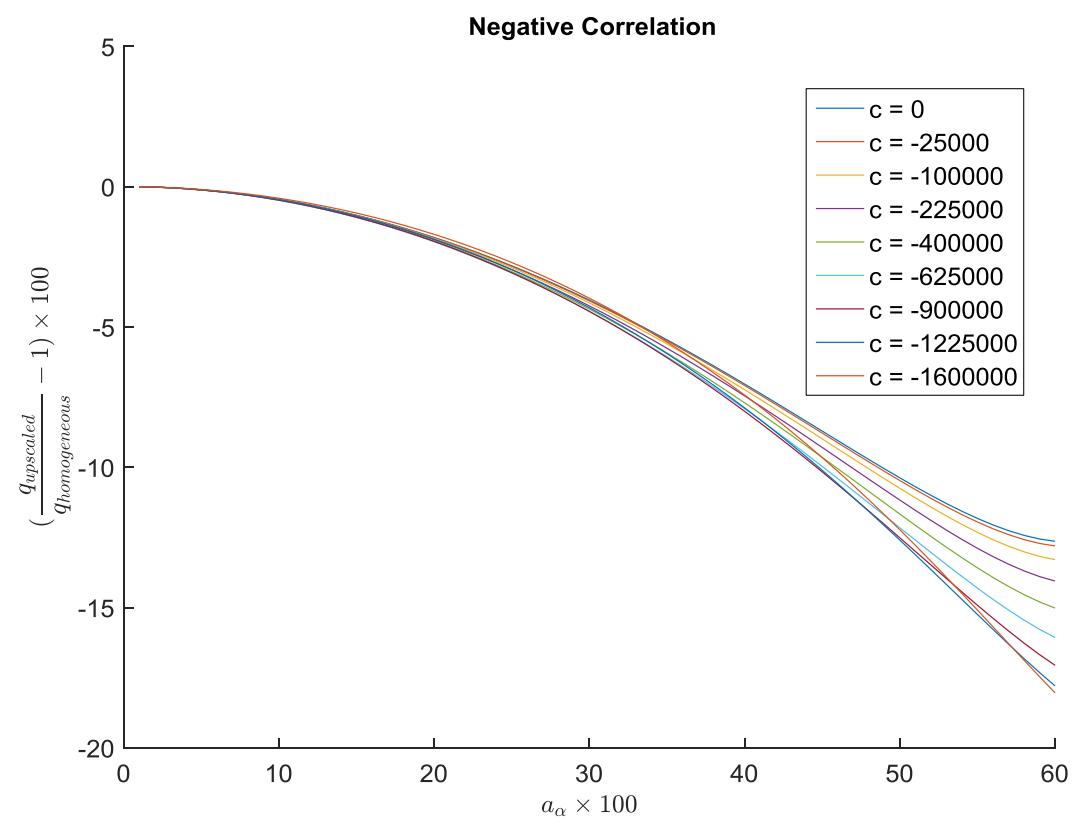

Figure 5-10: Effect of heterogeneities on the flow rate against constant values of c for negative correlation 


\subsection{Uncorrelated Flow Rate Curves}

In In previous section, two types of correlation between heterogeneities was investigated. But in case that the heterogeneity in one of properties is negligibly small, the effect of the other property on the SAGD will be different. In Figure 5-11, it is assumed that there is no diffusivity perturbation in the reservoir. It is seen that increasing the permeability variations, reduces the upscaled flow rate. For the opposite case, where the reservoir bears only perturbations of diffusivity, as seen in Figure 5-12, increasing the diffusivity perturbations, decreases the flow rate much more than the same size perturbations in permeability does as shown in Figure 5-11.

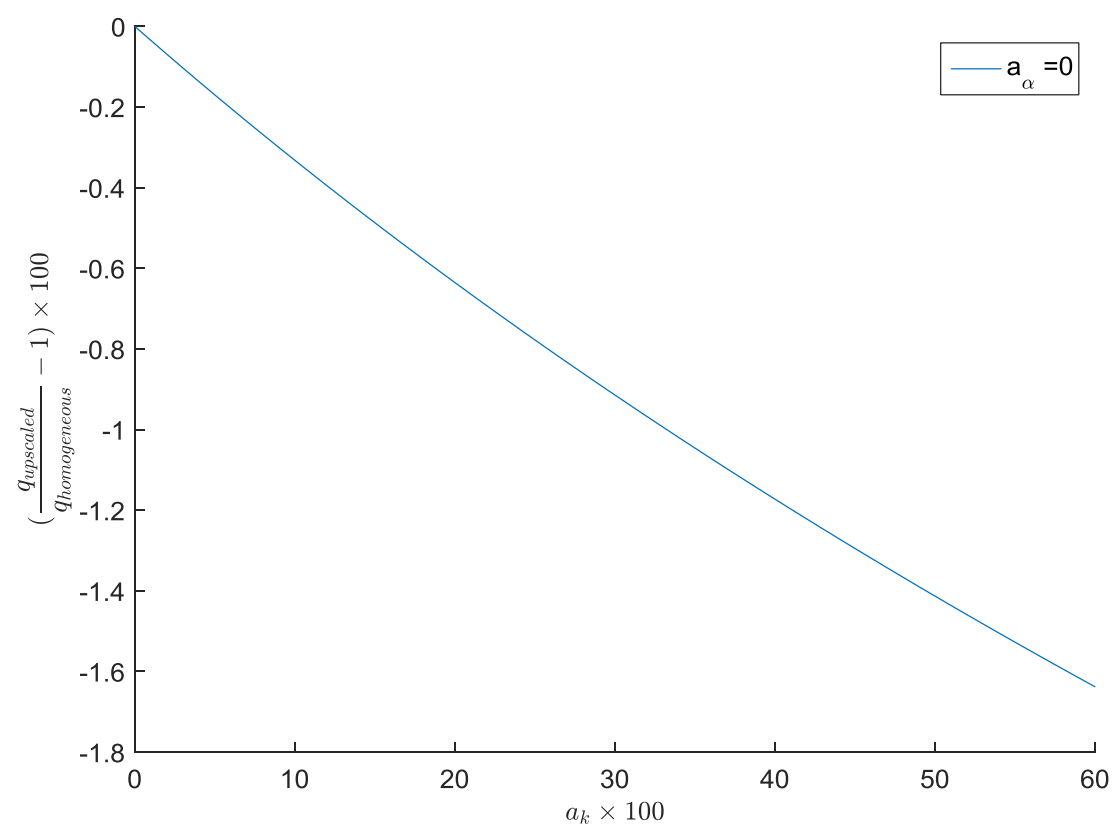

Figure 5-11: Effect of permeability heterogeneities on flow rate with no thermal diffusivity perturbation 


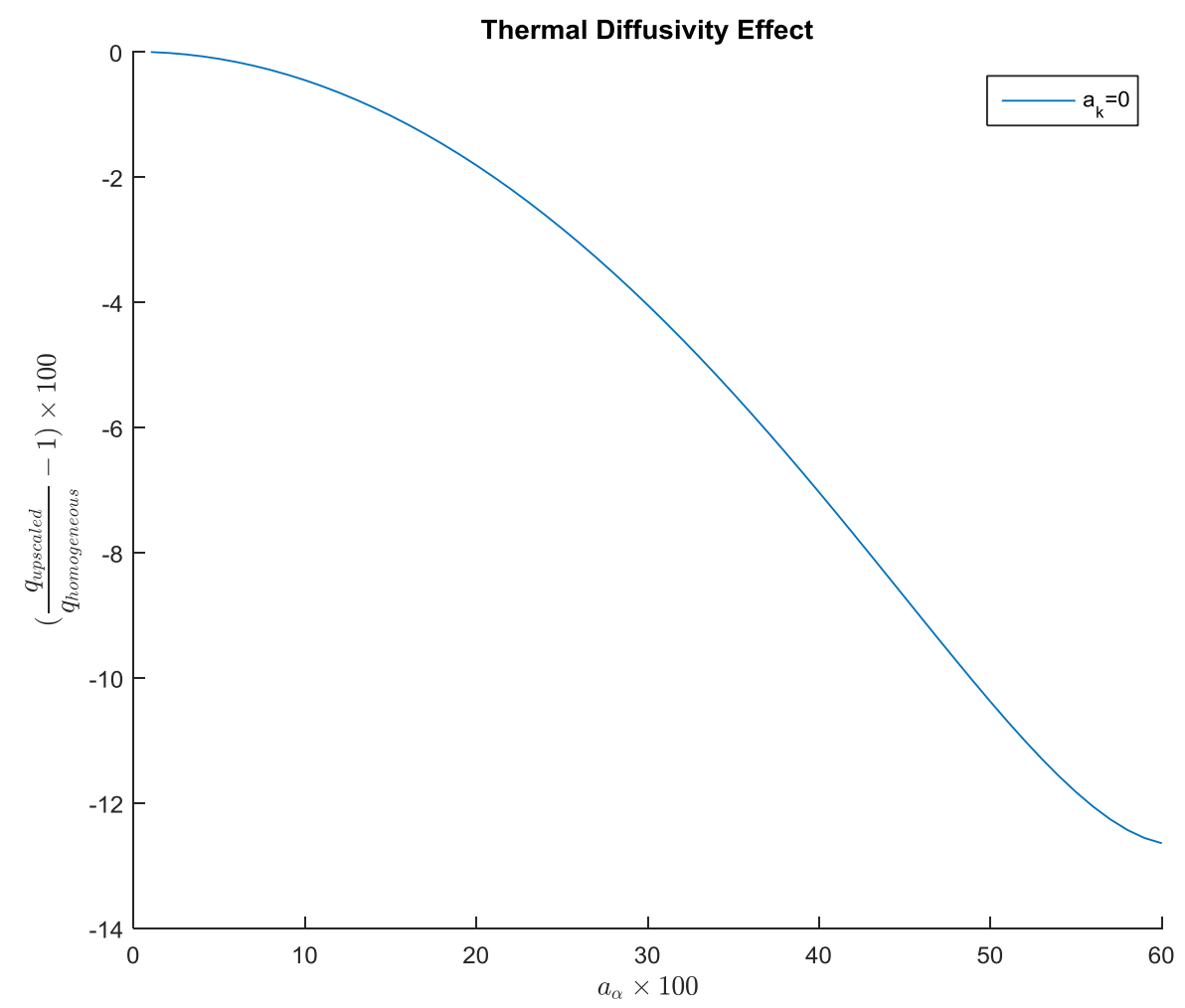

Figure 5-12: Effect of diffusivity heterogeneities on flow rate with no thermal permeability perturbation 


\subsection{Position of Interface}

As previously discussed, the interface of the boundary in SAGD satisfies following equations during its traveling through the reservoir.

$$
\begin{gathered}
Y=1-1 / 2\left(\frac{t^{\prime}}{X}\right)^{2} \\
Y=\frac{y}{h} \\
X=\frac{x}{h} \\
t^{\prime}=\frac{t}{h} \sqrt{\left(-\frac{\left(\bar{\alpha}-\frac{a^{2}}{\bar{\alpha}}\right) \frac{K_{L} g}{V_{R}}}{h * \bar{\phi} \Delta S_{o} * A A}\right)}
\end{gathered}
$$

To compare the heterogeneity effects on the velocity of boundary with homogeneous reservoir, parameter time ratio is defined:

$$
\text { time }_{\text {ratio }}=\frac{t_{\text {upscaled }}^{\prime}}{t_{\text {homogenous }}^{\prime}}=\sqrt{\left(\frac{\left(\bar{\alpha}-\frac{a^{2}}{\bar{\alpha}}\right)}{A A_{\text {upscaled }}} \times \frac{A A_{\text {homogeneous }}}{\bar{\alpha}}\right)}
$$

time $_{\text {ratio }}$ represents the relative speed of boundary compared to homogeneous reservoir. In Figure 5-13, the calculated location curves have been drawn for different values of t'. In Figure 5-14, time ratio values $\left(\right.$ time $_{\text {ratio }}$ ) for different heterogeneities have been shown. If this parameter is greater than one, it indicates that the boundary has slowed down in comparison with homogenous reservoir. 


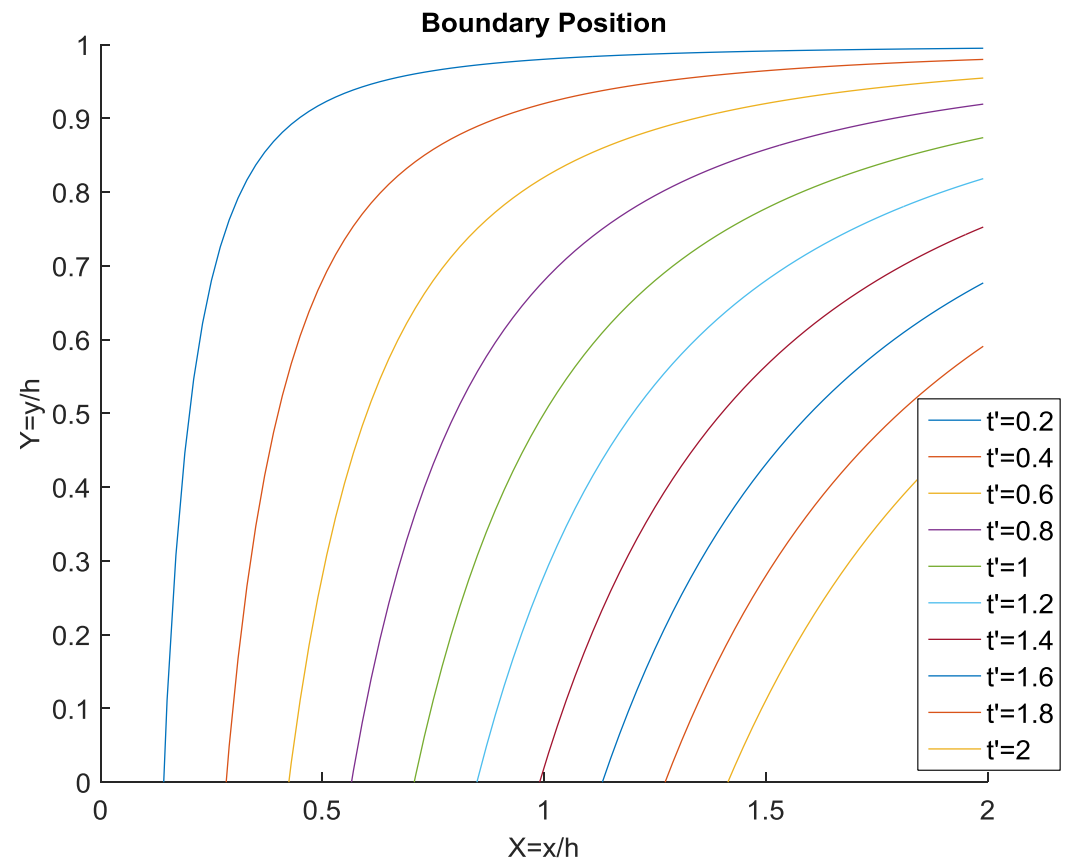

Figure 5-13: Calculated interface curves

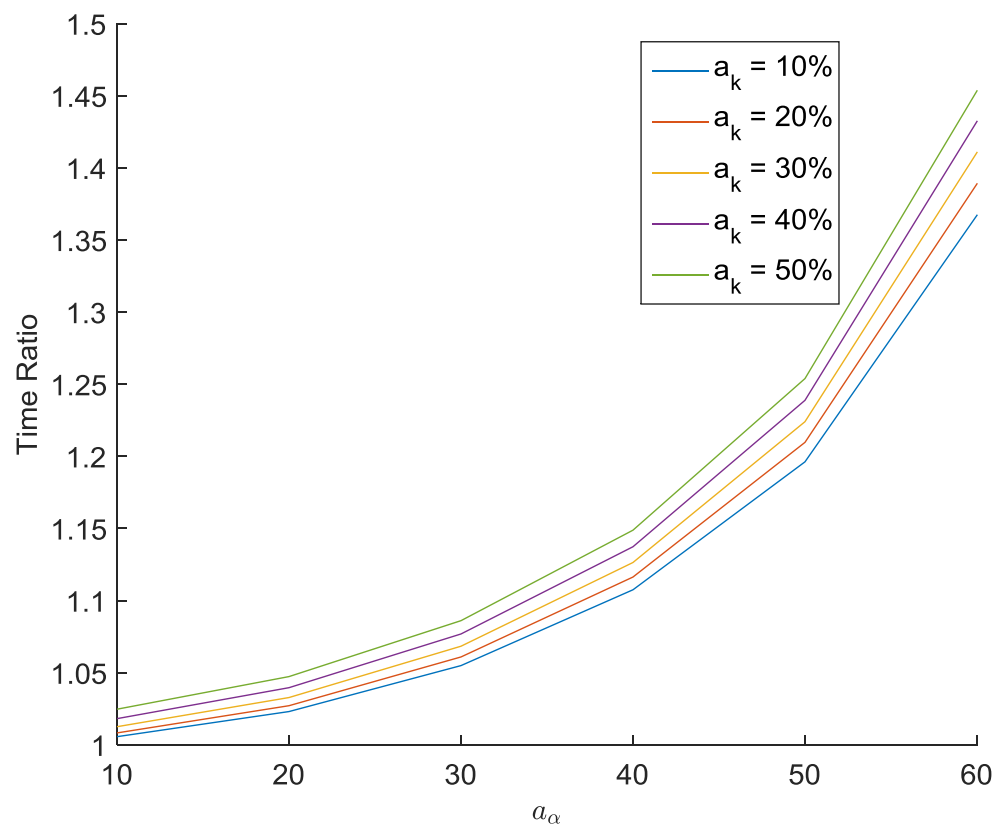

Figure 5-14: Effect of heterogeneities on the interface curves 


\section{Conclusion}

The results of this work reveal the important role of thermal diffusivity in SAGD process. Most of previous studies were concentrated on permeability but this study shows that from the prospect of heterogeneity, thermal diffusivity is more influential.

In addition, the developed model in this research showed that heterogeneities in permeability do not necessarily have an adverse effect on SAGD but even in some specific cases it may boost the efficiency of the recovery of heavy oil.

Another important conclusion that can be derived from this study indicates that heterogeneities in one property cannot be seen separately from heterogeneities in other properties. As seen, permeability variations always inhibit the SAGD and reduce the flowrate but when reservoir has also thermal diffusivities, in some specific conditions permeability heterogeneities turn out to improve the functionality of SAGD by increasing the drainage rate. 


\section{Recommendation of Future Work}

In this research, the heterogeneities of properties were simulated with harmonic correlation functions. Other types of heterogeneities such as shale streaks can be considered in the next step for the models developed based on this work.

In addition, the vertical and horizontal permeability have been assumed identical in development of this model. A correctional modification can be considered with assigning different values to this parameter so that it includes the directional anisotropy in the modeling as well.

They are other problems such as diversion or break through that may take place in practice but have not been considered in analytical model. The developed model may be the best choice in the initial steps of a project but to get the best result in next steps, it is recommended using this model along with a numerical-based software so that higher accuracy is guaranteed in the modeling. 


\section{References}

[1] Al-Bahlani, Al-Muatasim, and Tayfun Babadagli. "SAGD laboratory experimental and numerical simulation studies: A review of current status and future issues." Journal of Petroleum Science and Engineering 68.3 (2009): 135-150.

[2] Butler, Roger M. "Thermal recovery of oil and bitumen." 1991.

[3] Jose Walter Prada Vanegas, Clayton Vernon Deutsch, Luciane Bonet Cunha, et al. Uncertainty assessment of SAGD performance using a proxy model based on butler's theory. In SPE Annual Technical Conference and Exhibition. Society of Petroleum Engineers, 2008.

[4] Rose P.E. The steam assisted gravity drainage of oil sand bitumen. 1993.

[5] Sharma, Bakul C., Santanu Khataniar, Shirish L. Patil, Vidyadhar A. Kamath, and Abhijit Y. Dandekar. "A simulation study of novel thermal recovery methods in the ugnu tar sand reservoir, North Slope, Alaska." In SPE Western Regional/AAPG Pacific Section Joint Meeting. Society of Petroleum Engineers, 2002.

[6] Butler, R. M., Q. Jiang, and C. T. Yee. "Steam and gas push (SAGP)-4; Recent theoretical developments and laboratory results using layered models. "Journal of Canadian Petroleum Technology 40.01 (2001).

[7] Robinson, Bill, et al. "Geostatistical Modeling Integral to Effective Design and Evaluation of SAGD Processes of an Athabasca Oilsands Reservoir, a Case Study." SPE International Thermal Operations and Heavy Oil Symposium. Society of Petroleum Engineers, 2005.

[8] Larter, S., et al. "The origin, prediction and impact of oil viscosity heterogeneity on the production characteristics of tar sand and heavy oil reservoirs." Journal of Canadian Petroleum Technology 47.01 (2008).

[9] Chen, Qing. Assessing and improving steam-assisted gravity drainage: Reservoir heterogeneities, hydraulic fractures, and mobility control foams. 2009.

[10] Peaceman, D.W. Reperesentation of a Horizontal Well in Numerical Reservoir Simulation. ., 1993 SPE Advanced Technology Series 1.

[11] Alali, Najeh, Mahmoud Reza Pishvaie, and Hadi Jabbari. "A new semi-analytical modeling of steam-assisted gravity drainage in heavy oil reservoirs. "Journal of Petroleum Science and Engineering 69.3 (2009): 261-270. 
[12] Azom, Prince Nnamdi, and Sanjay Srinivasan. "Modeling the effect of permeability anisotropy on the steam-assisted gravity drainage (SAGD) process." Canadian Unconventional Resources Conference. Society of Petroleum Engineers, 2011.

[13] Kumar, Dhananjay. Modeling steam assisted gravity drainage in heterogeneous reservoirs using different upscaling techniques. Diss. 2014.

[14] Durlofsky, Louis J. "Numerical calculation of equivalent grid block permeability tensors for heterogeneous porous media." Water resources research 27, no. 5 (1991): 699-708.

[15] Gelhar, L. W. "Stochastic analysis of phreatic aquifers." Water Resources Research 10, no. 3 (1974): 539-545.

[16] Freeze, R. Allan. "A stochastic-conceptual analysis of one-dimensional groundwater flow in nonuniform homogeneous media." Water Resources Research 11, no. 5 (1975): 725-741.

[17] Bakr, Adel A., Lynn W. Gelhar, Allan L. Gutjahr, and John R. MacMillan. "Stochastic analysis of spatial variability in subsurface flows: 1 . Comparison of one-and three-dimensional flows." Water Resour. Res 14, no. 2 (1978): 263-271.

[18] Welty, C., and L. W. Gelhar. "Stochastic analysis of the effects of fluid density and viscosity variability on macrodispersion in heterogeneous porous media: Water Resour ResV27, N8, Aug 1991, P2061-2075." International Journal of Rock Mechanics and Mining Sciences \& Geomechanics Abstracts. Vol. 29. No. 2. Pergamon, 1992.

[19] Gelhar, Lynn W. Stochastic subsurface hydrology. Prentice-Hall, 1993. Chapter 1-4.

[20] Alali, Najeh, Mahmoud Reza Pishvaie, and Hadi Jabbari. "A new semi-analytical modeling of steam-assisted gravity drainage in heavy oil reservoirs. "Journal of Petroleum Science and Engineering 69.3 (2009): 261-270.

[21] Azom, Prince Nnamdi, and Sanjay Srinivasan. "Modeling the effect of permeability anisotropy on the steam-assisted gravity drainage (SAGD) process." Canadian Unconventional Resources Conference. Society of Petroleum Engineers, 2011.

[22] Kumar, Dhananjay. Modeling steam assisted gravity drainage in heterogeneous reservoirs using different upscaling techniques. Diss. 2014.

[23] Biercuk, M. J., Mark C. Llaguno, M. Radosavljevic, J. K. Hyun, Alan T. Johnson, and John E. Fischer. "Carbon nanotube composites for thermal management." Applied physics letters 80, no. 15 (2002): 2767-2769. 
[24] Chung, K. H and Butler, R. M. : "A Theoretical and Experimental Study of SAGD Process, “paper \#89, Proc. 4 th UNITAR/UNDP International Conference om Heavy Crude and Tar Sands, Edmonton, Canada, Aug 7-12,1988, p. 191-210

[25] Glandt, C. A., and Hsu Chia-Fu. "Electric Preheating in Low-Injectivity Tar Sand Deposits." In SPE/DOE Enhanced Oil Recovery Symposium. Society of Petroleum Engineers, 1992.

[26] Brooks, Robert Thomas, and Habib Habib Tavakol. "Experiences in Eliminating Steam Breakthrough and Providing Zonal Isolation in SAGD Wells." In SPE Heavy Oil Conference Canada. Society of Petroleum Engineers, 2012.

[27] Azad, Ali, and Richard Chalaturnyk. "Application of analytical proxy models in reservoir estimation for SAGD process: UTF-project case study." Journal of Canadian Petroleum Technology 52, no. 03 (2013): 219-232.

[28] Popov, Yuri, Vladimir Tertychnyi, Raisa Romushkevich, Dmitri Korobkov, and Jean Pohl. "Interrelations between thermal conductivity and other physical properties of rocks: experimental data." In Thermo-Hydro-Mechanical Coupling in Fractured Rock, pp. 1137-1161. Birkhäuser Basel, 2003.

[29] Mielke, P., G. Bignall, and I. Sass. "Permeability and Thermal Conductivity Measurements of Near Surface Units at the Wairakei Geothermal Field, New Zealand." In Proceedings of the World Geothermal Congress 2010. 2010. 